\title{
Stellar atmospheric parameters of FGK-type stars from high-resolution optical and near-infrared CARMENES spectra
}

\author{
E. Marfil ${ }^{\oplus},{ }^{\star}{ }^{\star}$ H. M. Tabernero, ${ }^{2,3}$ D. Montes ${ }^{\oplus},{ }^{1}$ J. A. Caballero, ${ }^{2}$ M. G. Soto, ${ }^{4}$ \\ J. I. González Hernández, ${ }^{5,6}$ A. Kaminski, ${ }^{7}$ E. Nagel, ${ }^{8}$ S. V. Jeffers, ${ }^{9}$ A. Reiners, ${ }^{9}$ \\ I. Ribas, ${ }^{10,11}$ A. Quirrenbach ${ }^{7}$ and P. J. Amado ${ }^{12}$ \\ ${ }^{1}$ Facultad de Ciencias Físicas, Departamento de Física de la Tierra y Astrofísica \& IPARCOS-UCM (Instituto de Física de Partículas y del Cosmos de la \\ UCM), Universidad Complutense de Madrid, E-28040 Madrid, Spain \\ ${ }^{2}$ Centro de Astrobiología (CSIC-INTA), ESAC, Camino Bajo del Castillo s/n, E-28691 Villanueva de la Cañada, Madrid, Spain \\ ${ }^{3}$ Instituto de Astrofísica e Ciências do Espaço, Universidade do Porto, CAUP, Rua das Estrelas, P-4150-762 Porto, Portugal \\ ${ }^{4}$ School of Physics and Astronomy, Queen Mary, University of London, 327 Mile End Rd., E1 4NS London, UK \\ ${ }^{5}$ Departamento de Astrofísica, Universidad de La Laguna, E-38206 La Laguna, Tenerife, Spain \\ ${ }^{6}$ Instituto de Astrofísica de Canarias, vía Láctea s/n, E-38205 La Laguna, Tenerife, Spain \\ ${ }^{7}$ Landessternwarte, Zentrum für Astronomie der Universität Heidelberg, Königstuhl 12, D-69117 Heidelberg, Germany \\ ${ }^{8}$ Hamburger Sternwarte, Gojenbergsweg 112, D-21029 Hamburg, Germany \\ ${ }^{9}$ Institut für Astrophysik, Georg-August-Universität-Göttingen, Friedrich-Hund-Platz 1, D-37077 Göttingen, Germany \\ ${ }^{10}$ Institut de Ciències de l'Espai (CSIC), Campus UAB, C/ de Can Magrans s/n, E-08193 Cerdanyola del Vallès, Spain \\ ${ }^{11}$ Institut d'Estudis Espacials de Catalunya (IEEC), C/ Gran Capità 2-4, E-08034 Barcelona, Spain \\ ${ }^{12}$ Instituto de Astrofísica de Andalucía (IAA-CSIC), Glorieta de la Astronomía s/n, E-18008 Granada, Spain
}

Accepted 2019 December 28. Received 2019 December 9; in original form 2019 October 4

\begin{abstract}
With the purpose of assessing classic spectroscopic methods on high-resolution and high signal-to-noise ratio spectra in the near-infrared wavelength region, we selected a sample of 65 F-, G-, and K-type stars observed with CARMENES, the new, ultra-stable, doublechannel spectrograph at the $3.5 \mathrm{~m}$ Calar Alto telescope. We computed their stellar atmospheric parameters $\left(T_{\text {eff }}, \log g, \xi\right.$, and $\left.[\mathrm{Fe} / \mathrm{H}]\right)$ by means of the STEPAR code, a PYTHON implementation of the equivalent width method that employs the 2017 version of the MOOG code and a grid of MARCS model atmospheres. We compiled four Fe I and Fe II line lists suited to metal-rich dwarfs, metal-poor dwarfs, metal-rich giants, and metal-poor giants that cover the wavelength range from 5300 to $17100 \AA$, thus substantially increasing the number of identified Fe I and $\mathrm{Fe}$ II lines up to 653 and 23, respectively. We examined the impact of the near-infrared $\mathrm{Fe}$ I and Fe II lines upon our parameter determinations after an exhaustive literature search, placing special emphasis on the 14 Gaia benchmark stars contained in our sample. Even though our parameter determinations remain in good agreement with the literature values, the increase in the number of $\mathrm{Fe} \mathrm{I}$ and $\mathrm{Fe}$ II lines when the near-infrared region is taken into account reveals a deeper $T_{\text {eff }}$ scale that might stem from a higher sensitivity of the near-infrared lines to $T_{\text {eff }}$.
\end{abstract}

Key words: line: identification - techniques: spectroscopic - stars: fundamental parameters stars: solar-type-infrared: stars.

\section{INTRODUCTION}

The homogeneous, automated computation of stellar atmospheric parameters from stellar spectra, i.e. effective temperature $T_{\text {eff }}$, surface gravity $\log g$, stellar metallicity $[\mathrm{M} / \mathrm{H}]$, and micro-turbulent velocity $\xi$, plays a crucial role in many astrophysical contexts. First, it leads to the analysis of the fundamental properties of individual

^E-mail: emigom01@ucm.es objects as well as of large stellar samples (Valenti \& Fischer 2005; Adibekyan et al. 2014). In this regard, large stellar spectroscopic surveys such as RAVE (Steinmetz et al. 2006), APOGEE (Allende Prieto et al. 2008), the Gaia-ESO Survey (Gilmore et al. 2012), and GALAH (De Silva et al. 2015) have laid the foundations for our current understanding of the structure and evolution of the Milky Way. Secondly, exoplanetary studies also rely on stellar parameter determinations not only to enable the determination of both planetary radii and masses (e.g. Mann et al. 2019; Schweitzer et al. 2019) but also to characterize the habitable zones around 
planet-harbouring stars (Kasting, Whitmire \& Reynolds 1993; Kopparapu et al. 2013). Furthermore, correlations between the stellar metallicity and planet occurrence rates are now well established and shed light on planet formation mechanisms (Adibekyan et al. 2014; Delgado Mena et al. 2018; Montes et al. 2018).

The equivalent width (EW) method (see e.g. Sousa et al. 2008; Tabernero, Montes \& González Hernández 2012; Mucciarelli et al. 2013; Tsantaki et al. 2013; Bensby, Feltzing \& Oey 2014; Andreasen et al. 2016) is, along with the spectral synthesis method (see e.g. Valenti \& Fischer 2005; Piskunov \& Valenti 2017), one of the most widely used spectroscopic techniques for determining stellar atmospheric parameters. A full account of the key caveats of these two methods can be found in Jofré, Heiter \& Soubiran (2019) and Blanco-Cuaresma (2019). The advent of high-resolution nearinfrared (NIR) spectrographs such as CARMENES (Quirrenbach et al. 2018), SPIRou (Artigau et al. 2014), GIANO (Origlia et al. 2014; Oliva et al. 2018), CRIRES+ (Hatzes \& CRIRES + Team 2017), IRD (Kotani et al. 2014), HPF (Wright et al. 2018), and NIRPS (Wildi et al. 2017) allows us to revisit these techniques, originally applied in the optical, in order to assess the impact of the NIR wavelength range on stellar parameter computations. In this context, new observations of FGK-type stars carried out with CARMENES, ${ }^{1}$ the double-channel spectrograph at the $3.5 \mathrm{~m}$ Calar Alto telescope open up a unique opportunity to test the reliability of such techniques on high-resolution and high signal-to-noise $(\mathrm{S} / \mathrm{N})$ ratio spectra in the optical and near-infrared windows.

In this work, we compute the spectroscopic parameters of 65 FGK-type stars selected from a CARMENES stellar library by means of the EW method, which relies on the strength (i.e the EW measurements) of $\mathrm{Fe} \mathrm{I}$ and $\mathrm{Fe}$ II absorption lines to derive the stellar atmospheric parameters $T_{\text {eff }}, \log g,[\mathrm{Fe} / \mathrm{H}]$, and $\xi$ assuming local thermodynamic equilibrium. To do so, we followed the approach of Sousa et al. (2007) to automatically measure the EW of the iron lines, and the STEPAR code (Tabernero et al. 2019) to automatically compute the stellar atmospheric parameters imposing excitation and ionization equilibrium conditions on the Fe I and Fe II lines.

The wavelength coverage provided by CARMENES, from 5200 up to $17100 \AA$, allowed us to substantially increase the number of $\mathrm{Fe} I$ and $\mathrm{Fe}$ II lines subject to analysis with the EW method with respect to previous studies restricted to the optical window (Meléndez \& Barbuy 2009; Jofré et al. 2014). Furthermore, the high spectral resolution of CARMENES, which is $R=94600$ in the VIS channel and $R=80400$ in the NIR channel (Quirrenbach et al. 2018), significantly improves both the line identification process and the EW measurements. Despite the availability of iron line lists optimized for the NIR region in the literature, the impact on stellar parameter determinations of FGK-type stars is still unknown, mostly due to the fact that such line lists have not as yet been systematically applied to significantly large samples covering a wide portion of the stellar parameter space. For instance, Andreasen et al. (2016) compiled a line list of Fe I and Fe II lines in the region $10000-25000 \AA$, but only tested it against the spectra of the Sun and the F8 IV star HD 20010.

Several other spectral libraries of high-resolution spectra in the near-infrared have been developed over the past few years. For example, Lebzelter et al. (2012) presented the CRIRES-POP spectral library, which provides high-resolution $(R \sim 100000)$ spectra for 25 stars between B and M spectral types at 1-5 $\mu \mathrm{m}$. Furthermore, Nicholls et al. (2017) described the data reduction

${ }^{1}$ http : // carmenes. caha.es process and presented the first CRIRES-POP spectral atlas of the K giant 10 Leo. Although the resolution of the spectra in this library is comparable to that of CARMENES, the number of available spectra is significantly lower than the size of the library analysed in this work, and does not satisfactorily cover the parameter space of FGK-type stars. Another example is the IGRINS spectral library (Park et al. 2018), which contains spectra of 84 stars between $\mathrm{O}$ and M spectral types in the $H(1.49-1.80 \mu \mathrm{m})$ and $K(1.96-2.46 \mu \mathrm{m})$ bands with a resolution of $R=45000$, which is almost half of that provided by CARMENES in the NIR channel. Finally, large surveys such as APOGEE (Zamora et al. 2015; Majewski et al. 2017) have obtained intermediate-resolution $(R \sim 22500)$ spectra for hundreds of thousands of stars, but with a narrow wavelength coverage in the $H$ band $(1.5-1.7 \mu \mathrm{m})$.

The analysis performed in this work is structured as follows. In Section 2, we describe the selection of the sample. In Section 3, we outline the main steps of our analysis, including the line selection process and the workflow of the STEPAR code. In Sections 4 and 5, we discuss the results and highlight the conclusions, respectively.

\section{SAMPLE}

We observed an extensive sample of dwarf, giant, and supergiant stars and brown dwarfs with spectral types from $\mathrm{O} 4$ to late $\mathrm{L}$ as part of the first open time proposal that used CARMENES. While further details on this stellar library will be provided in forthcoming publications (Caballero et al. in preparation), we start here its scientific exploitation.

From the stellar library we selected 65 stars with spectral types later than F5 and earlier than K4, and projected equatorial rotational velocities $v \sin i<15 \mathrm{~km} \mathrm{~s}^{-1}$ (see Table A1). The restriction in spectral type stems from the general limitations of the EW method and hence, STEPAR, as explained in Tabernero et al. (2019), while stars with high rotational velocities have line profiles that cannot be properly fitted by a Gaussian shape, leading to less reliable EW measurements. None of the observed 65 FGK-type stars had a known visual (physical) or optical (non-physical) companion at less than 5 arcsec. However, we excluded from this analysis one of the giants found in the library, c Gem, with spectral type K4.5 III (Keenan \& McNeil 1989), as it appeared as an SB2 binary system after cross-correlating its spectrum with the atlas spectrum of Arcturus, as explained in Section 3.1.

Our target list contains 14 Gaia benchmark stars (Jofré et al. 2014, 2018; Heiter et al. 2015), including the Sun. The spectrum of the Sun was obtained through the observation of the asteroid 1 Ceres due to the allocation of Calar Alto Director's discretionary time. According to their original purpose, the fact that the fundamental parameters of these stars have been computed independently from spectroscopy makes them suitable as a reference to assess any method aimed at the automated analysis of cool stars.

Table A1 displays the star names, Henry-Draper numbers, equatorial coordinates from 2MASS (Skrutskie et al. 2006), parallaxes from the Gaia Data Release 2 (Gaia Collaboration 2018) if available, and the Hipparcos mission (van Leeuwen 2007), along with the spectral types, the values of $T_{\text {eff }}, \log g, \xi,[\mathrm{Fe} / \mathrm{H}]$ and the stellar projected rotational velocities, $v \sin i$, found in the literature for the selected sample. For the Gaia benchmark stars, we adopted the parameters from Jofré et al. (2014) and Heiter et al. (2015), with updated values from Jofré et al. (2018). For the remaining stars, we tabulate the stellar parameters from the most recent references found in the PASTEL catalogue (Soubiran et al. 2016). 


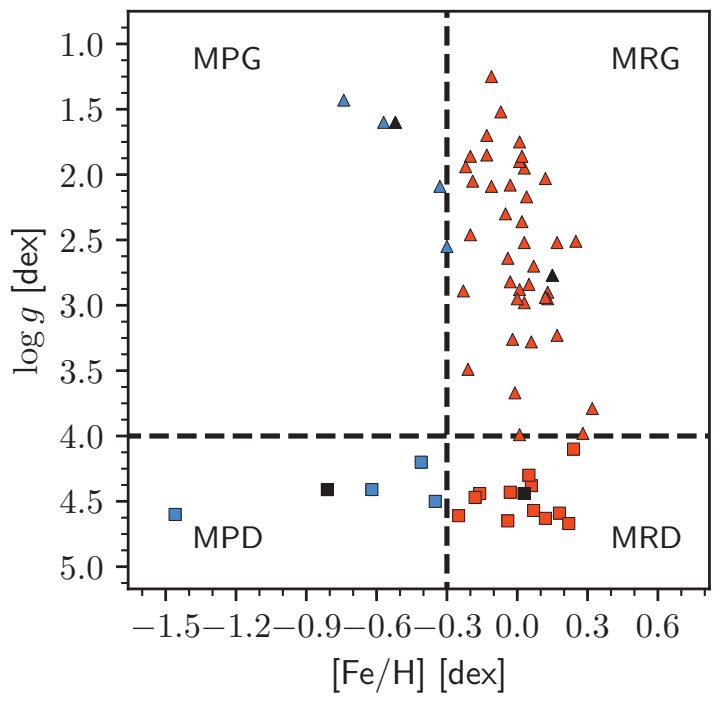

Figure 1. Division of the parameter space in the sample according to the stellar atmospheric parameters found in the literature. The vertical and horizontal dashed black lines represent the boundaries at $[\mathrm{Fe} / \mathrm{H}]=-0.3$ dex and $\log g=4.0 \mathrm{dex}$, respectively, for metal-rich dwarfs (MRDs, orange squares), metal-poor dwarfs (MPDs, blue squares), metal-rich giants (MRGs, orange triangles), and metal-poor giants (MPGs, blue triangles). The stars taken as a reference for each of these regions are shown in black.

Following Tabernero et al. (2019), we divided the parameter space into four different regions in terms of $\log g$ and $[\mathrm{M} / \mathrm{H}]$, using $[\mathrm{Fe} / \mathrm{H}]$ as a proxy of stellar metallicity, in order to simplify our search for iron lines in the CARMENES spectra, as explained in Section 3.2. We thus made a distinction between the dwarf regime, $\log g \geq 4.00$, and the giant regime, $\log g<4.00$, and between metal-rich stars, $[\mathrm{Fe} / \mathrm{H}]>-0.30$, and metal-poor stars, $[\mathrm{Fe} / \mathrm{H}] \leq-0.30$. We dubbed the four resulting line lists metal-rich dwarfs (MRDs), metal-poor dwarfs (MPDs), metal-rich giants (MRGs), and metal-poor giants (MPGs). We selected the following Gaia benchmark stars, all of which were observed with CARMENES, as a reference for the assembly of the corresponding Fe I and Fe II line lists: 18 Sco for the MRD, $\mu$ Cas for the MPD, $\epsilon$ Vir for the MRG, and Arcturus for the MPG. We show this division of the parameter space in Figs 1 and 2.

\section{ANALYSIS}

\subsection{Data processing}

The 65 pairs of VIS and NIR spectra were taken in service mode between 2016 March and 2016 June with the two CARMENES channels operating simultaneously. In general, exposure times were manually adjusted to reach an S/N between 100 and 300 in the $J$ band. The observations were carried out without the simultaneous wavelength calibration of the Fabry-Pérot etalons since there was no particular interest in precise radial velocity determinations (i.e. better than $\sim 20 \mathrm{~m} \mathrm{~s}^{-1}$ ) for these stars.

The spectra were taken in 'target + sky' mode, i.e. the stars were observed in fibre A and the sky in fibre B. Both fibres are identical but fibre B is located at 88 arcsec to the east. Star and sky spectra are available through the Calar Alto archive. In our work, we did not subtract the corresponding sky spectrum to each star spectrum, as this is an ongoing analysis (Nagel et al. in preparation).

The raw spectra were reduced with the CARACAL pipeline (Zechmeister, Anglada-Escudé \& Reiners 2014; Caballero et al.

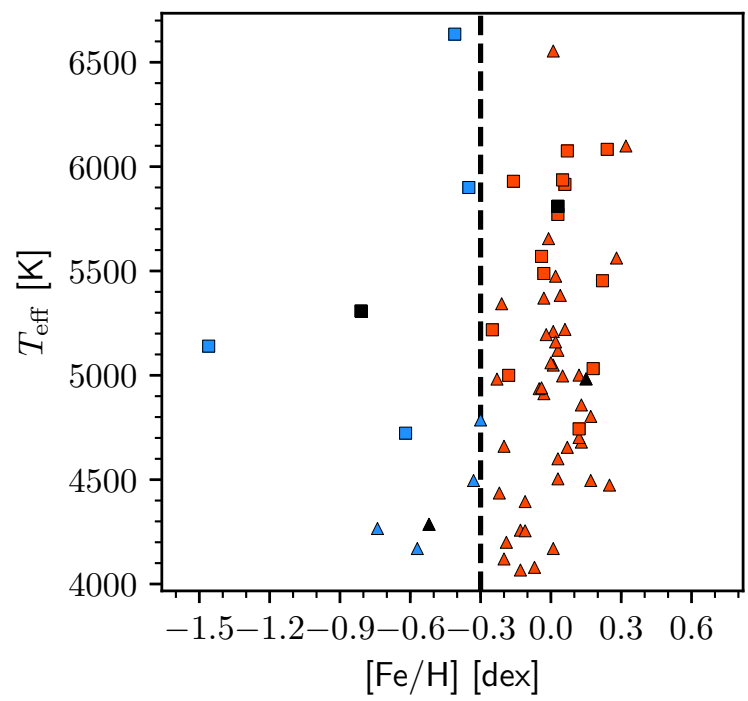

Figure 2. Same as Fig. 1, but for literature values of $T_{\text {eff }}$ versus $[\mathrm{Fe} / \mathrm{H}]$ in the sample. Only the boundary at $[\mathrm{Fe} / \mathrm{H}]=-0.3 \mathrm{dex}$ is shown.
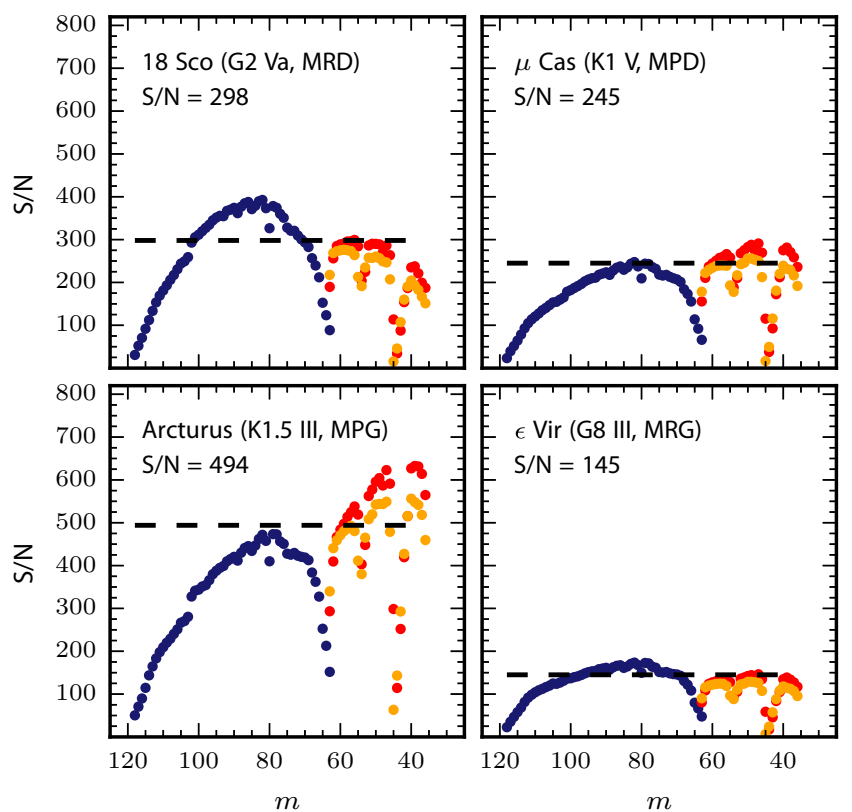

Figure 3. CARACAL $\mathrm{S} / \mathrm{N}$ of the CARMENES spectra of the reference stars (18 Sco, $\mu$ Cas, $\epsilon$ Vir, and Arcturus) as a function of the spectral order $m$. The blue circles are the orders in the VIS channel, while the orange and red circles are the two $\mathrm{HgCdTe}$ array detectors of the NIR channel. The dashed black lines mark the global S/N estimation given by iSpec.

2016), which is based on the IDL REDUCE package (Piskunov \& Valenti 2002). CARACAL generates one fully reduced, wavelengthcalibrated, one-dimensional spectrum of the individual spectral orders. Fig. 3 displays the CARACAL $\mathrm{S} / \mathrm{N}$ of the four reference spectra as a function of the diffraction order $m$. We estimated the global $\mathrm{S} / \mathrm{N}$ of the spectra with the integrated Spectroscopic framework (iSpec, see Blanco-Cuaresma et al. 2014) in terms of the median of the flux values divided by their corresponding flux errors. The global $\mathrm{S} / \mathrm{N}$ of the selected spectra can also be found in Table A2. 


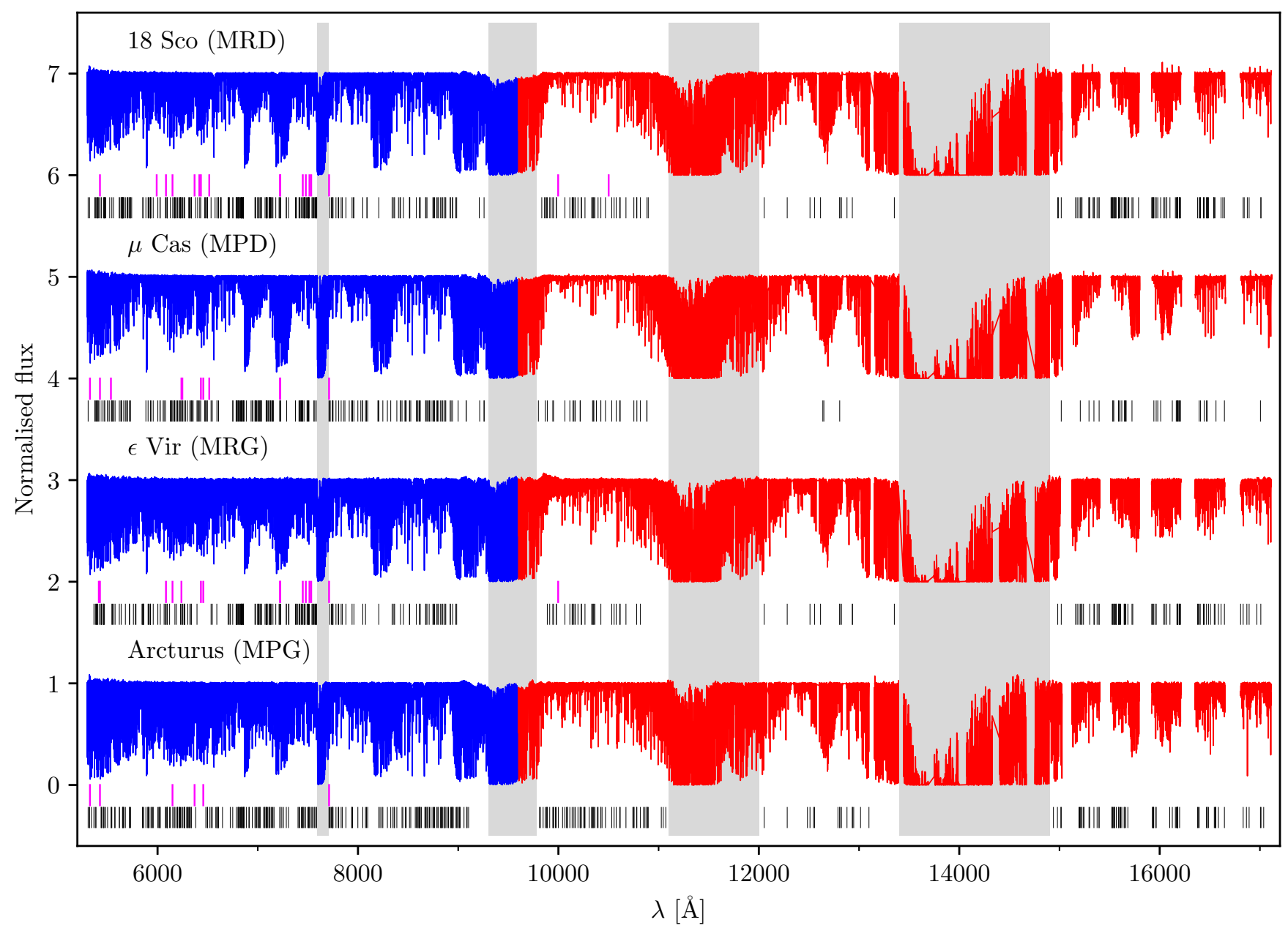

Figure 4. Distribution of the selected Fe I and Fe II absorption lines in the reference spectra. The Fe I and Fe II lines are shown as black and pink vertical lines, respectively, below the spectra. The VIS and NIR channels of the CARMENES instrument are shown in blue and red, respectively. The grey shaded areas show the regions severely affected by telluric absorption.

Next, we employed a wavelength grid to merge the spectral orders of both channels into one single spectrum. The wavelength grid, which is evenly spaced on a logarithmic scale, mirrors the natural wavelength spacing of the CARMENES spectrographs across the orders. In Fig. 4, we show the normalized, merged spectra of the four stars taken as a reference in this work.

Since the CARMENES instrument operates in vacuum, we performed a vacuum-to-air wavelength conversion of the order-merged, channel-merged, CARMENES spectra to provide the wavelengths of the Fe I and $\mathrm{Fe}$ II lines on an air scale, following the International Astronomical Union standard (Morton 2000):

$\lambda_{\text {air }}=\frac{\lambda_{\text {vacuum }}}{n}$,

where $n$ is the refraction index, which is given by the following expression:

$n=1+8.34254 \times 10^{-5}+\frac{2.406147 \times 10^{-2}}{130-s^{2}}+\frac{1.5998 \times 10^{-4}}{38.9-s^{2}}$,

where $s=10^{4} / \lambda_{\text {vacuum }}$, with $\lambda_{\text {vacuum }}$ in $\AA$.

After the vacuum-to-air wavelength conversion, we accounted for the barycentric velocity of the observatory at the time of observations. We then computed the radial velocities with iSpec by means of the cross-correlation function between the observed
CARMENES spectra and a template spectrum provided by iSpec in the following way. In the dwarf regime, we set as the template a solar spectrum based on data from the NARVAL (Aurière 2003) and HARPS (Mayor et al. 2003) instruments (see Blanco-Cuaresma et al. 2014) covering the overlap region with CARMENES, i.e. the 5200-10 $480 \AA$ range. Likewise, in the giant regime we set as the template spectrum an atlas of Arcturus covering the 5200$9260 \AA$ range (Hinkle et al. 2000). Both template spectra were corrected from telluric absorption features, which makes them suitable for cross-correlation. This allowed us to correct the spectra from the corresponding Doppler shift. In Fig. 5, we compare the radial velocities thus computed against the literature values. Four stars exhibit a difference in radial velocity greater than $1 \mathrm{~km} \mathrm{~s}^{-1}$ compared to literature values. These are all single-lined (SB1) spectroscopic binaries: $\mu$ Cas (Worek \& Beardsley 1977), $\alpha \mathrm{CMi}$ (Girard et al. 2000), $\alpha$ UMa (Spencer Jones \& Furner 1937), and $\zeta$ Her (Scarfe et al. 1983). The radial velocities of our sample can also be found in Table A2. The average difference in the computed radial velocities of the sample with respect to the literature values is $0.09 \pm 0.64 \mathrm{~km} \mathrm{~s}^{-1}$.

\subsection{Fe I and Fe II line selections}

We requested four line lists from the Vienna Atomic Line Database (VALD3; Piskunov et al. 1995; Kupka et al. 2000, 


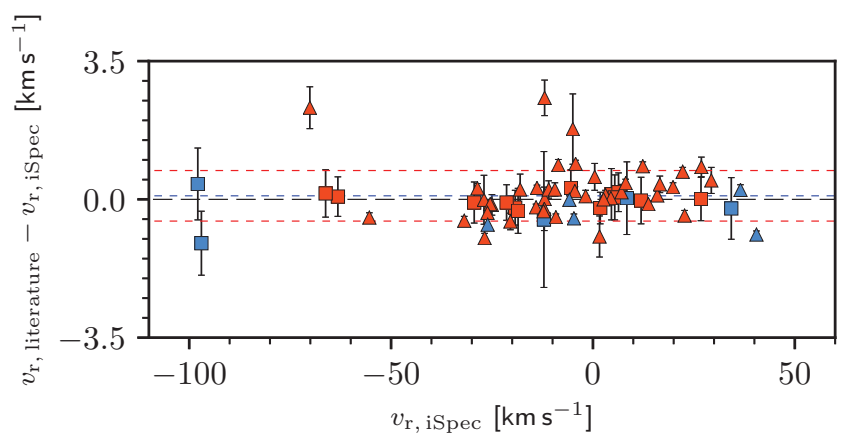

Figure 5. Comparison between the radial velocities $v_{\mathrm{r}}$ of the sample obtained with iSpec and the literature values. Symbols are the same as in Fig. 1. The dotted blue and red lines are the average difference and the corresponding $1 \sigma$ dispersion, respectively.

1999; Ryabchikova et al. 2015), corresponding each to one of our four reference spectra. We used the option Extract stellar available at the VALD3 website, ${ }^{2}$ with a wavelength range from 5300 to $17100 \AA$, a minimum line depth of 5 per cent with respect to the continuum flux, and the corresponding input stellar parameters found in Table A1. We excluded the wavelength range 5200-5300 from this search because of the low $\mathrm{S} / \mathrm{N}$ of the CARMENES spectra in this region.

Because of its user-friendly interface, we used iSpec to select the Fe I and Fe II spectral lines by visually projecting the VALD3 line list files on to the corresponding processed reference spectra. We rejected $\mathrm{Fe}$ I and $\mathrm{Fe}$ II lines that showed spectral blending with close atomic and molecular lines. Since telluric lines are ubiquitous in the near-infrared and at the red end of the optical (see e.g. Reiners et al. 2018), we computed a synthetic transmission spectrum via the telluric-correction tool molecfit (Kausch et al. 2015; Smette et al. 2015), which makes use of the line-by-line radiative transfer model (LBLRTM, Clough et al. 2005) and the HITRAN molecular line data base (Gordon et al. 2017), to model the Earth's atmospheric transmission spectrum. This allowed us to prevent wrong line identification throughout the visual inspection of the reference spectra. Further details on the telluric correction of the CARMENES spectra can be found in Passegger et al. (2019). A full description of the correction will appear in a forthcoming publication of the CARMENES series (Nagel et al. in preparation).

To expedite our analysis, we also looked for $\mathrm{Fe}$ I and Fe II line compilations found in the literature that overlap with the wavelength range covered by CARMENES. Since the careful analysis of the optical wavelength range up to $\sim 6860 \AA$ has already led to several line lists published in previous works that were specifically compiled to yield the best possible set of stellar atmospheric parameters for FGK-type stars (see e.g. Sousa et al. 2008; Jofré et al. 2014; Tabernero et al. 2019), we refrained from further refining the line selection in this window and adopted the iron lines given in Sousa et al. (2008). As to the near-infrared region, we checked our iron line selections from 10000 to $17100 \AA$ against the ones tabulated in Andreasen et al. (2016). Despite our careful search for Fe II in the NIR region, we only found one Fe II line at $\lambda=10501.503 \AA$. Finally, iron lines found in the region 6800-10000 $\AA$ were not compared with the literature due to the lack of line compilations in this spectral window. In Table 1, we show a summary of the number of iron lines listed in this work on a global and per-line list basis, i.e.

${ }^{2}$ http: //vald.astro.uu.se
Table 1. Number of Fe I and Fe II lines reported in this work, Sousa et al (2008, Sou08), Andreasen et al. (2016, And16), and Tabernero et al. (2019, Tab19), from 5300 to $17100 \AA$.

\begin{tabular}{llcc}
\hline Reference & \multicolumn{2}{c}{ Line list/region } & \multicolumn{2}{c}{ \#lines } \\
& & Fe I & Fe II \\
\hline This work & MRD & 386 & 16 \\
This work & MPD & 295 & 9 \\
This work & MRG & 306 & 13 \\
This work & MPG & 379 & 4 \\
This work & CARMENES VIS channel & 437 & 21 \\
This work & CARMENES NIR channel & 216 & 2 \\
This work & Globally & 653 & 23 \\
Tab19 & MRD & 112 & 8 \\
Tab19 & MPD & 82 & 8 \\
Tab19 & MRG & 72 & 7 \\
Tab19 & MPG & 95 & 5 \\
Tab19 & Globally & 175 & 14 \\
Sou08 & - & 172 & 19 \\
And16 & - & 272 & 12 \\
\hline
\end{tabular}

MRD, MPD, MRG, and MPG, in comparison with those tabulated in Sousa et al. (2008) and Andreasen et al. (2016) in the wavelength region covered by CARMENES.

Since we assembled the line lists considering four specific reference spectra, we removed the Fe I and Fe II line identifications that fall into any of the CARMENES inter- and intra-order gaps ${ }^{3}$ as a consequence of the corresponding Doppler shift corrections in the remaining spectra of the sample.

In Fig. 4, we show the distribution of the selected Fe I and Fe II lines in the reference spectra. In addition, in Fig. A1 we give a closeup view of the spectrum of the reference, solar-type star 18 Sco along with the line selections. We give the central wavelength in air, $\lambda_{\text {air }}$, the excitation potential, $\chi$, and the oscillator strength, $\log g f$, of the selected Fe I and Fe II lines in Tables A4 and A5, respectively.

\subsection{EW measurements}

We computed the EWs by fitting Gaussian profiles to the absorption lines, ${ }^{4}$ as shown in Fig 6. First, we selected a region approximately $6 \AA$ wide centred at the selected absorption line, $l$, and performed a continuum normalization on the spectra following Sousa et al. (2007). Specifically, we fitted a third-degree polynomial to the data, selecting only the points that lie within rejt times the polynomial, where $r e j t=1-1 /(\mathrm{S} / \mathrm{N})$, and $\mathrm{S} / \mathrm{N}$ is the signal-to-noise ratio of the region. We then identified the absorption lines present in the spectra by finding the points where the first derivative of the data was zero, and the second derivative was positive. Finally, we fitted Gaussian profiles to the lines detected, and integrated the profile corresponding to the selected line $l$ to obtain the EW. The uncertainty in the EW was estimated by changing the Gaussian parameter estimates within $1 \sigma$ of their uncertainty for a total of 1000 iterations, and looking at the EW distribution.

As in Tabernero et al. (2019), we only considered lines with $10 \mathrm{~m} \AA<\mathrm{EW}<120 \mathrm{~m} \AA$ for all stars in the sample to avoid problems with line profiles of very intense lines and potentially bad EW measurements of extremely weak lines.

\footnotetext{
${ }^{3}$ http://carmenes.caha.es/ext/instrument/

${ }^{4}$ The code is available at:https://github.com/msotov/EWComp utation
} 

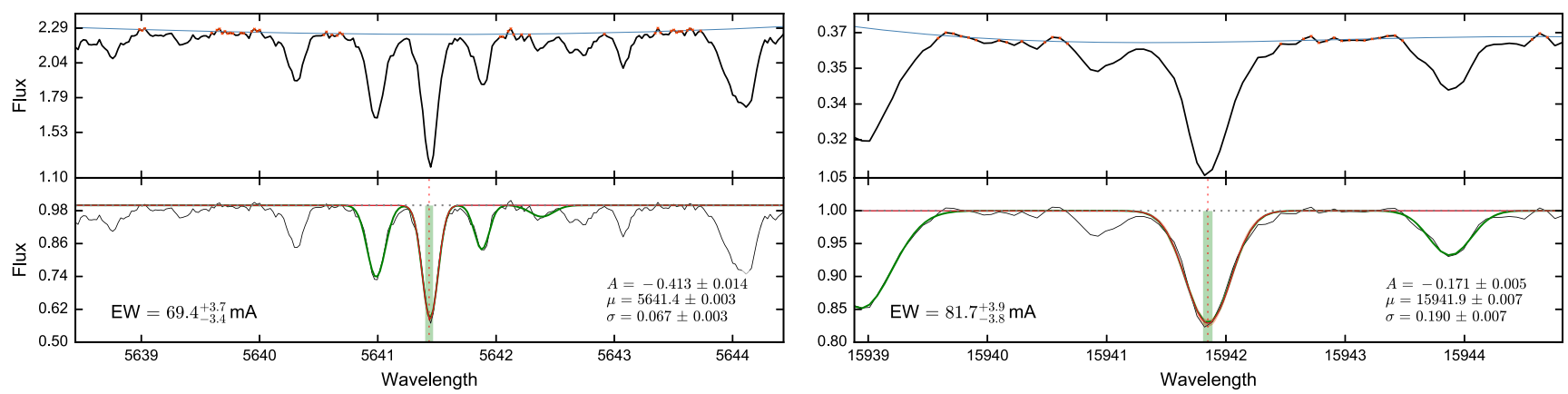

Figure 6. EW measurements of two Fe I lines in the spectrum of $18 \mathrm{Sco}$, at $5641.434 \AA$ (left) and $12824.859 \AA$ (right). The upper panels illustrate the continuum determination, where the points used for the final polynomial fit are highlighted in red. The bottom panels show the full fit performed for all detected lines, shown in green, and the Gaussian fit of the selected line, shown in red, parametrized by the central intensity in normalized units, $A$, the central wavelength in $\AA, \mu$, and the Gaussian dispersion, $\sigma$. The shaded red area depicts the $1 \sigma$ confidence intervals of the Gaussian fit, and the green square, the $E W$ estimation, as explained in the text.

\subsection{STEPAR}

The STEPAR code ${ }^{5}$ is a PYTHON implementation of the EW method specifically designed for the automated and simultaneous computation of the stellar atmospheric parameters of FGK-type stars, namely $T_{\text {eff }}, \log g,[\mathrm{Fe} / \mathrm{H}]$, and $\xi$. STEPAR is one of the 13 pipelines in the Gaia-ESO Survey used in the analysis of UVES U580 spectra of late-type, low-mass stars. A full description of its workflow and performance can be found in Tabernero et al. (2019). STEPAR is an iterative code that derives the stellar parameters and their associated uncertainties by imposing both excitation and ionization equilibrium conditions on a set of Fe I and Fe II lines, using the 2017 version of the MOOG $^{6}$ code (Sneden 1973) and a grid of plane-parallel and spherical MARCS ${ }^{7}$ model atmospheres (Gustafsson et al. 2008).

For any given MOOG-compliant EW input file comprised of a significant number of Fe I and Fe II lines, STEPAR follows a Downhill Simplex minimization algorithm (Press et al. 2002) across the parameter space in order to find the stellar atmospheric parameters that best reproduce the observed EWs. The code takes $T_{\text {eff }}=5777 \mathrm{~K}$, $\log g=4.44 \mathrm{dex}$, and $\xi=1.0 \mathrm{~km} \mathrm{~s}^{-1}$ as the initial input values.

If we let $\epsilon(\mathrm{Fe})$ represent the iron abundance retrieved from any given $\mathrm{Fe}$ line and $\chi$ be the excitation potential of the line, STEPAR iterates until the slopes of $\chi$ versus $\log \epsilon(\mathrm{Fe} \mathrm{I})$ and $\log \mathrm{EW} / \lambda$ versus $\log \epsilon(\mathrm{Fe} \mathrm{I})$ are zero, i.e. the iron atoms are in excitation equilibrium. It also imposes ionization equilibrium so that $\log \epsilon(\mathrm{Fe} I)$ $=\log \epsilon(\mathrm{Fe}$ II $)$. Throughout this iterative process, the code verifies that the average $[\mathrm{Fe} / \mathrm{H}]$ in the MOOG output is always compatible with the iron abundance of the input atmospheric model. Next, STEPAR performs an individual $\sigma$ clipping on the Fe I and Fe II lines to remove the ones that imply an iron abundance, $\log \epsilon(\mathrm{Fe})$, that exceeds the $3 \sigma$ limit with respect to the median abundance of all lines. After this step, STEPAR restarts the minimization algorithm with the remaining Fe I and Fe II lines, taking as initial input values the parameters computed in the first run. STEPAR computes the uncertainties in the stellar atmospheric parameters following the sequence: $\delta \xi, \delta T_{\text {eff }}, \delta \log g$, and $\delta[\mathrm{Fe} / \mathrm{H}]$. This computation relies on the retrieved Fe I and Fe II abundances and the uncertainties in the slopes that define the equilibria conditions. The code also propagates

\footnotetext{
${ }^{5}$ STEPAR is available at:https://github.com/hmtabernero/ste Par

${ }^{6}$ https://www.as.utexas.edu/ chris/moog.html

${ }^{7}$ http: //marcs.astro.uu.se
}

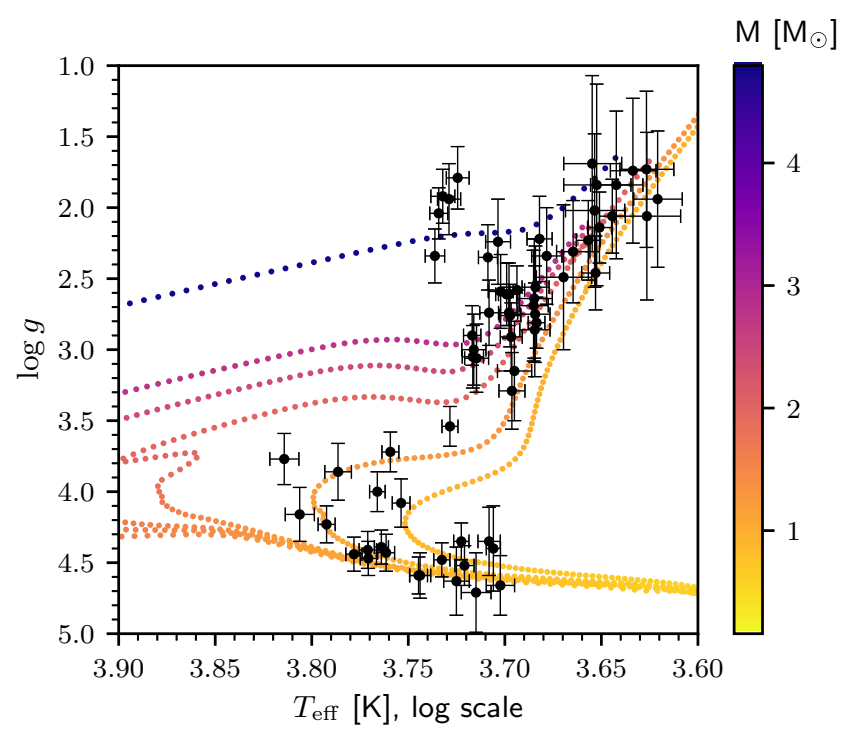

Figure 7. Kiel diagram ( $\log g$ versus $\left.\log T_{\text {eff }}\right)$ of the sample along with the YaPSI isochrones at $0.1,0.4,0.6,1,4$, and $13 \mathrm{Ga}$ (for $Z=0.016$, see Spada et al. 2017).

the uncertainties following the previous sequence. For example, the uncertainty in $[\mathrm{Fe} / \mathrm{H}]$ is a quadrature between the standard deviation of the Fe I and Fe II abundances and the propagated uncertainties in the remaining stellar parameters. Further details on the computation of the uncertainties can be found in Tabernero et al. (2019).

\section{RESULTS AND DISCUSSION}

In Table A2, we give the stellar atmospheric parameters of the sample computed with STEPAR. These were obtained after matching the corresponding Fe I and Fe II line lists to the stars according to their reference parameters reported in Table A1.

We also performed the analysis of the sample with the $E W$ method taking into account only the Fe I and Fe II lines found in the optical region covered by the VIS channel of the CARMENES instrument. The parameters thus obtained can be found in Table A3. Unfortunately, we could not attempt to analyse the NIR in the same manner because of the scarcity of Fe II lines above $9600 \AA$.

In Fig. 7, we display a Kiel diagram, i.e. $\log g$ versus $\log T_{\text {eff }}$, of our sample as computed with STEPAR, along with the Yale-Potsdam 


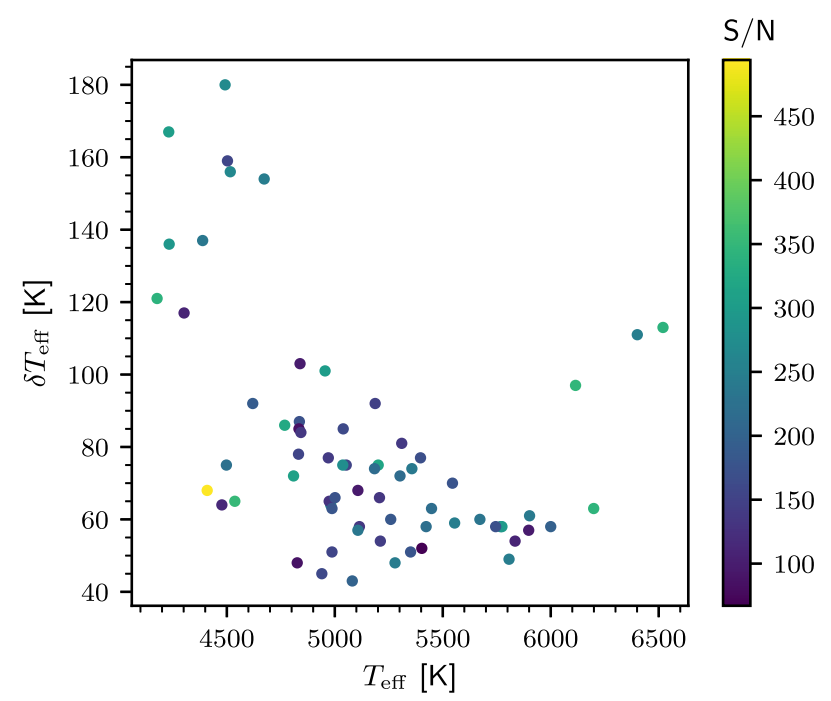

Figure 8. Uncertainties in $T_{\text {eff }}, \delta T_{\text {eff }}$, versus $T_{\text {eff }}$ for our sample, as computed with STEPAR.

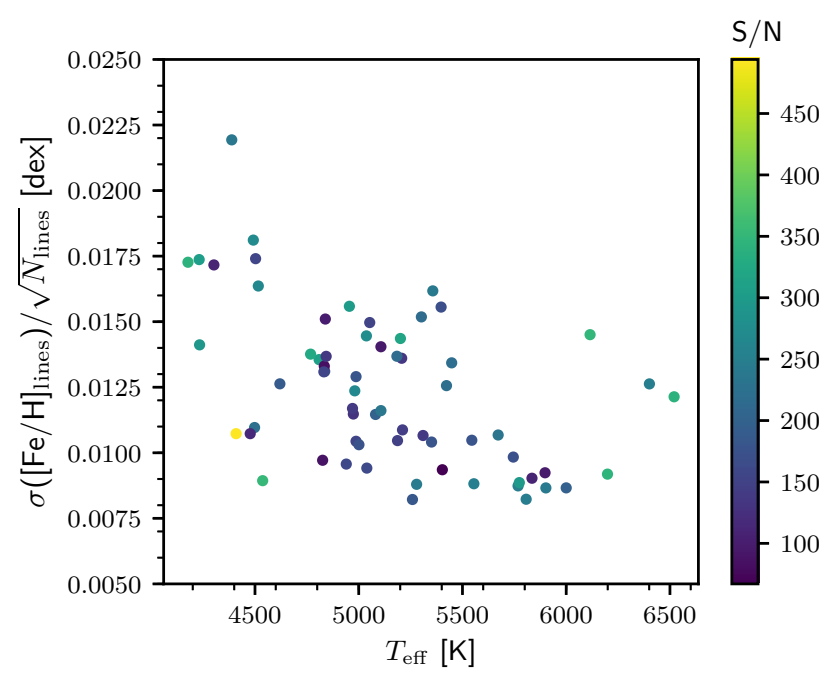

Figure 9. Line-to-line scatter in $[\mathrm{Fe} / \mathrm{H}]$ versus $T_{\text {eff }}$ and $\mathrm{S} / \mathrm{N}$ in the sample.

Stellar Isochrones (YaPSI, Spada et al. 2017) at solar metallicity, namely $Z=0.016$. Overall, we found no disparity between our derived values and the region of the parameter space covered by the isochrones. As pointed out by Tabernero et al. (2019), STEPAR returns slightly higher effective temperatures for F-type dwarfs. Five luminous, G-type, giant stars ( $\beta$ Dra, F Hya, $\epsilon$ Leo, $37 \mathrm{LMi}$, and $\zeta$ Mon) are located at an anomalous position in the Kiel diagram. According to Luck (2014), these stars are thought to be the evolved counterparts of early F- to B-type main-sequence stars that have reached the He-burning evolutionary stage.

In the cool regime, i.e. K-type stars, where stellar spectra become increasingly more crowded, the continuum placement is more uncertain, and the iron lines are subject to blending with other spectral features. On the other hand, sufficiently strong iron lines become increasingly scarce towards early F-type stars. This has a strong impact on the computed errors in the stellar atmospheric parameters, in particular the effective temperature, and the line-to-line scatter in $[\mathrm{Fe} / \mathrm{H}]$, as shown in Figs 8 and 9, respectively.
In Figs 10 and 11, we compare the stellar atmospheric parameters computed with STEPAR with values from the literature (McWilliam 1990; Heiter \& Luck 2003; Allende Prieto et al. 2004; Valenti \& Fischer 2005; Hekker \& Meléndez 2007; Liu et al. 2007; Sousa et al. 2008; Takeda, Sato \& Murata 2008; Lyubimkov et al. 2010; Wu et al. 2011; Thygesen et al. 2012; Santos et al. 2013; Jofré et al. 2014, 2015; Luck 2014; Morel et al. 2014; da Silva, Milone \& RochaPinto 2015; Jofré et al. 2018), taking into account the VIS and NIR channels simultaneously, and only the VIS channel, respectively. To explore possible sources of potential systematic trends or offsets, we followed the Monte Carlo method implemented in Tabernero et al. (2018). We generated 10000 synthetic samples based on our derived stellar atmospheric parameters. We computed all data points in each of these artificial samples by means of a normal distribution centred at the original measurements, and took the uncertainties in each parameter as the width of the distribution. The summary of the Monte Carlo simulations can be found in Table 2. We computed the Pearson and Spearman correlation coefficients, which quantify the degree of correlation between any two given variables. We found a significant correlation in the differences between our own $T_{\text {eff }}$ values and the literature versus the literature values. However, no such correlation was found in the derived $\log g$ and $[\mathrm{Fe} / \mathrm{H}]$ values.

At first glance, it seems that our temperature scale has an intrinsic systematic error with respect to the literature values. The offset appears to be linked to the fact that we now include the NIR channel, given that the correlation diminishes when we restrict the analysis to the iron lines found in the VIS channel. Although the STEPAR code could be thought to be the underlying reason for this correlation, we are not comparing the same temperature scale. In other words, we now take into account iron absorption lines in a wavelength region that is different from most studies found in the literature. In addition, this offset is more noticeable for the coolest stars. The former result could arise from the fact that the NIR lines are more sensitive to the effective temperature than the optical lines, at least for the cool stars. In other words, although the inclusion of the NIR in the analysis does not bring extreme differences of the derived stellar parameters with respect to the analysis using the optical range, it seems to reveal a deeper $T_{\text {eff }}$ scale as suggested by the meaningful correlation found in Table 2 as well as Figs 10 and 11.

In Fig. 12, we show the values of $\log g$ derived with STEPAR against those obtained adopting the distances from Gaia DR2 (Gaia Collaboration 2018), if available, and the Hipparcos mission (van Leeuwen 2007). We computed the latter $\log g$ values by means of the PARAM web interface ${ }^{8}$ (da Silva et al. 2006; Rodrigues et al. 2014, 2017), which employs a Bayesian approach to derive the stellar parameters, including stellar age, mass, and radius. The $\log g$ values obtained with PARAM can be found in Tables A2 and A3. Following the Monte Carlo method described above, we found a systematic offset of $0.15 \pm 0.38$ dex. The Pearson and Spearman correlation coefficients, which are $r_{\mathrm{p}}=-0.302 \pm 0.093$ and $r_{\mathrm{s}}=$ $0.259 \pm 0.104$, respectively, reveal a correlation of around 9 per cent, which is slightly lower than previous works (see e.g. Tabernero et al. 2017).

Regarding the micro-turbulent velocity, Fig. 13 shows the values of $\xi$ obtained with STEPAR against the literature. Our derived values for $\xi$ are compatible with the literature values to a large extent. However, six stars (i.e. $\beta$ Dra, F Hya, $\zeta$ Mon, $\sigma$ Oph, $\theta$ Her, and HD 77912), with computed $\xi$ values larger than $3 \mathrm{~km} \mathrm{~s}^{-1}$, show larger deviations with respect to the literature, which can be as large

${ }^{8}$ http: //stev.oapd.inaf.it/cgi-bin/param 

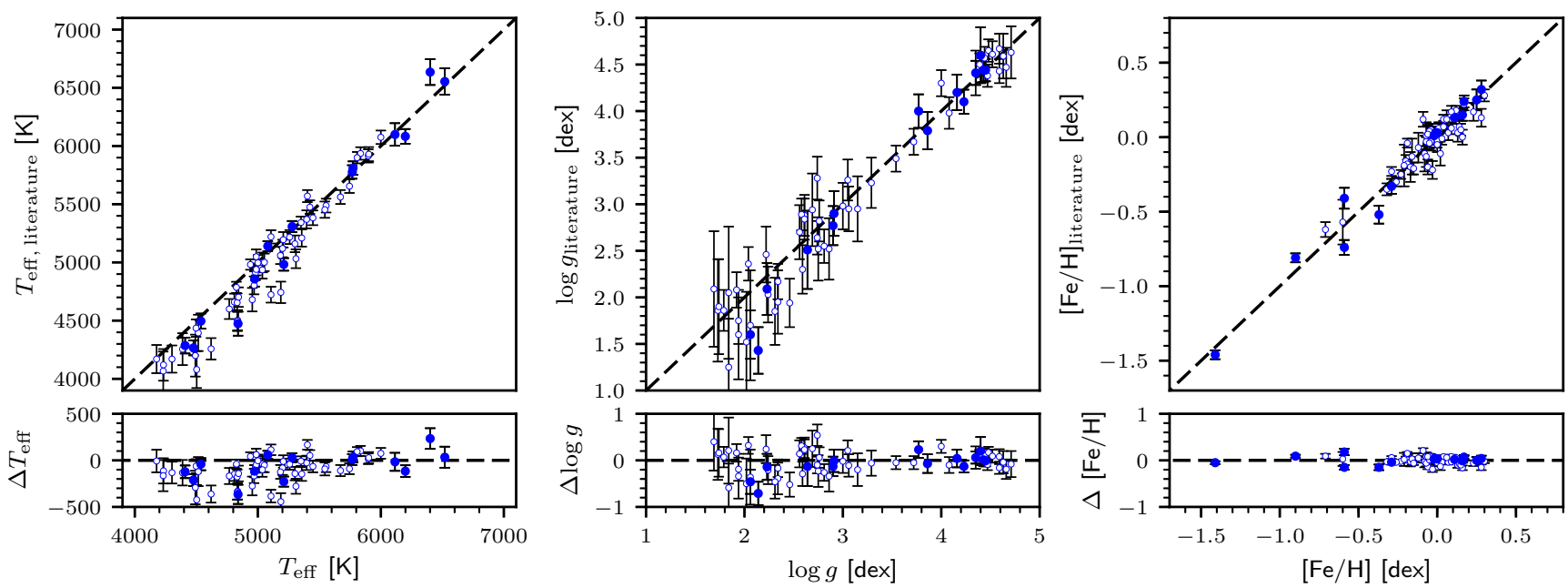

Figure 10. Comparison between the stellar atmospheric parameters obtained with STEPAR including the VIS and NIR channels of CARMENES and the literature values. The blue filled circles are the Gaia benchmark stars in our sample. The remaining stars in the sample are shown with the blue open circles. The dashed black lines indicate the one-to-one relationship. From left to right: $T_{\text {eff }}, \log g$, and [Fe/H].
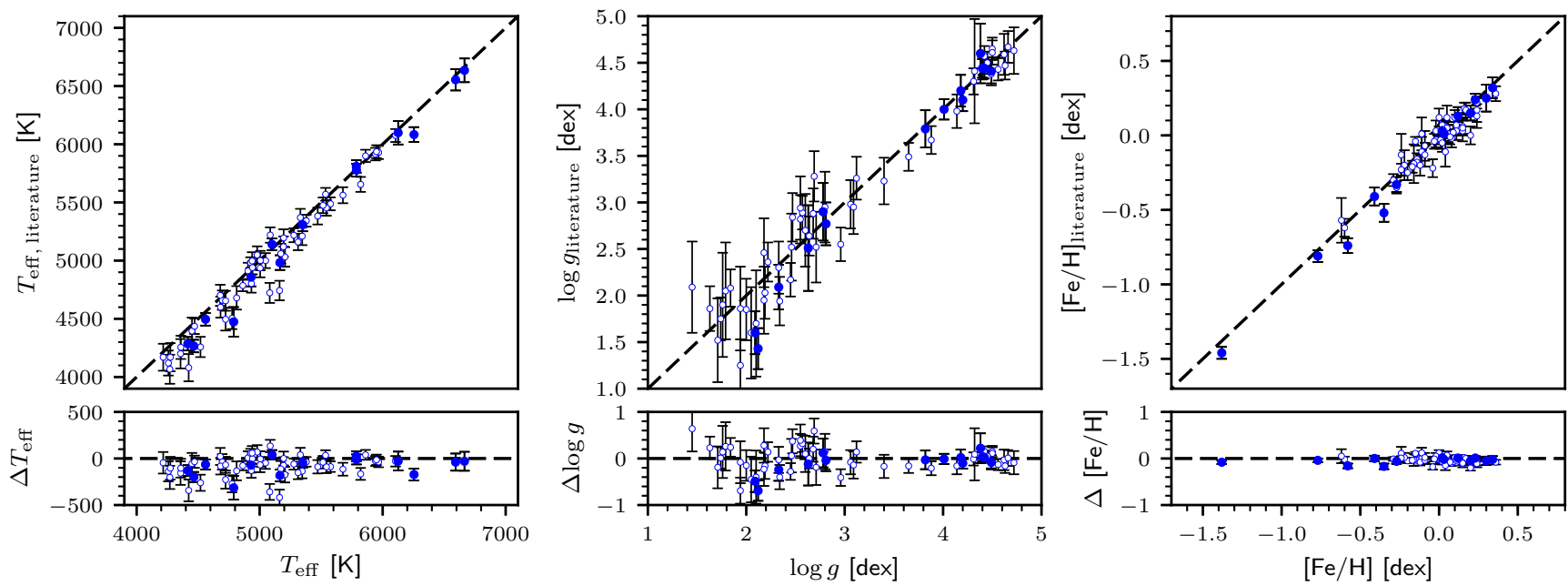

Figure 11. Same as Fig. 10 but restricting the analysis to the Fe I and Fe II lines found in the optical wavelength region covered by the VIS channel of CARMENES.

Table 2. Summary of the Monte Carlo simulations carried out on the $T_{\text {eff }}$, $\log g$, and $[\mathrm{Fe} / \mathrm{H}]$ values of the sample as computed with STEPAR. We show the average difference on each parameter and the values of the Pearson $\left(r_{\mathrm{p}}\right)$ and Spearman $\left(r_{\mathrm{s}}\right)$ correlation coefficients.

\begin{tabular}{lcrr}
\hline Parameter & \multicolumn{1}{c}{ Difference } & \multicolumn{1}{c}{$r_{\mathrm{p}}$} & \multicolumn{1}{c}{$r_{\mathrm{s}}$} \\
\hline \multicolumn{4}{c}{ VIS and NIR channels } \\
$T_{\text {eff }[\mathrm{K}]}$ & $-100 \pm 166$ & $0.40 \pm 0.07$ & $0.41 \pm 0.07$ \\
$\log g[\mathrm{dex}]$ & $-0.03 \pm 0.38$ & $0.10 \pm 0.10$ & $0.07 \pm 0.11$ \\
{$[\mathrm{Fe} / \mathrm{H}][\mathrm{dex}]$} & $0.00 \pm 0.11$ & $-0.09 \pm 0.06$ & $-0.12 \pm 0.07$ \\
\multicolumn{4}{c}{ VIS channel only } \\
$T_{\text {eff }}[\mathrm{K}]$ & $-92 \pm 135$ & $0.21 \pm 0.08$ & $0.21 \pm 0.09$ \\
$\log g[\mathrm{dex}]$ & $-0.01 \pm 0.38$ & $-0.01 \pm 0.10$ & $0.00 \pm 0.10$ \\
{$[\mathrm{Fe} / \mathrm{H}][\mathrm{dex}]$} & $-0.04 \pm 0.10$ & $-0.01 \pm 0.08$ & $-0.07 \pm 0.09$ \\
\hline
\end{tabular}

as $1.6 \mathrm{~km} \mathrm{~s}^{-1}$, as in the case of the star $\zeta$ Mon. In addition, we retrieved a significantly lower $\xi$ value for the star $v$ Boo compared to the literature. Although $\xi$ and $[\mathrm{Fe} / \mathrm{H}]$ are thought to be partially degenerate (Valenti \& Fischer 2005), we fail to identify the impact that such high or low $\xi$ values have on $[\mathrm{Fe} / \mathrm{H}]$ for these stars in our analysis. For example, a difference of $1.6 \mathrm{~km} \mathrm{~s}^{-1}$ in $\xi$ for the star $\zeta$ Mon leads to a difference of only $0.07 \mathrm{dex}$ in $[\mathrm{Fe} / \mathrm{H}]$ between the literature and the analysis with STEPAR, and both computed and literature values are compatible within error bars.

A closer look at the comparison between our parameter determinations and the Gaia benchmark star parameters from Heiter et al. (2015), with updated values from Jofré et al. (2018), can be found in Fig 14. We find good agreement between our derived values and the fundamental $T_{\text {eff }}$ and $\log g$, i.e. derived from the fundamental relations $L=4 \pi R^{2} \sigma T_{\text {eff }}^{4}$ and $g=G M / R^{2}$, respectively, by means of specific information that is available for these stars, such as the parallax, the angular diameter, and the bolometric flux. None the less, we note four outliers in $T_{\text {eff }}\left(\Delta T_{\text {eff }}>200 \mathrm{~K}\right)$ and two in $\log g$ 


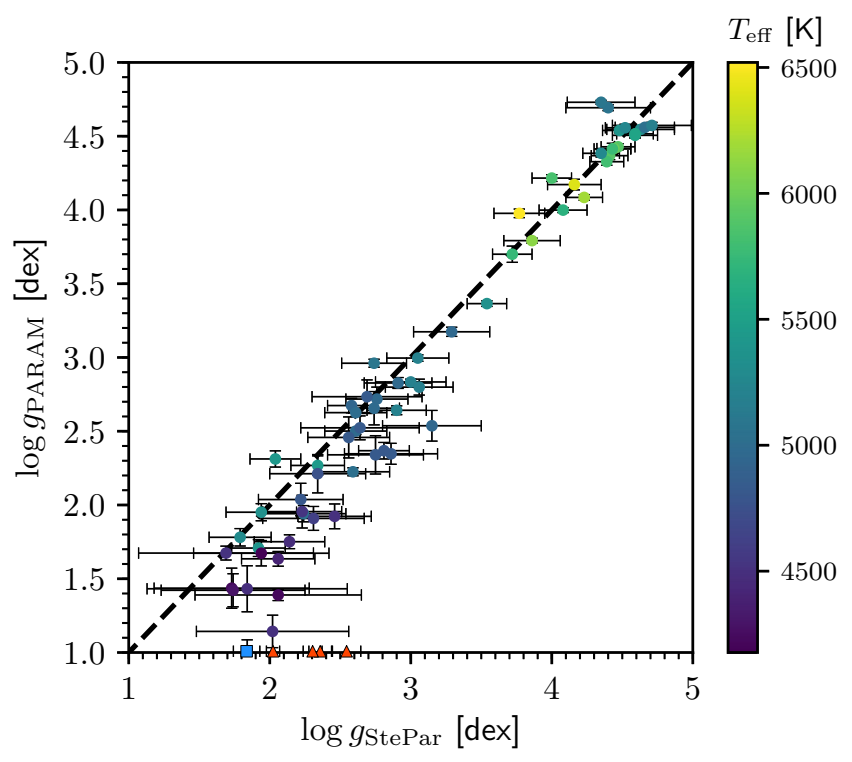

Figure 12. Surface gravities, $\log g$, derived for the sample with STEPAR versus those obtained with the code PARAM, adopting the distances from Gaia DR2.

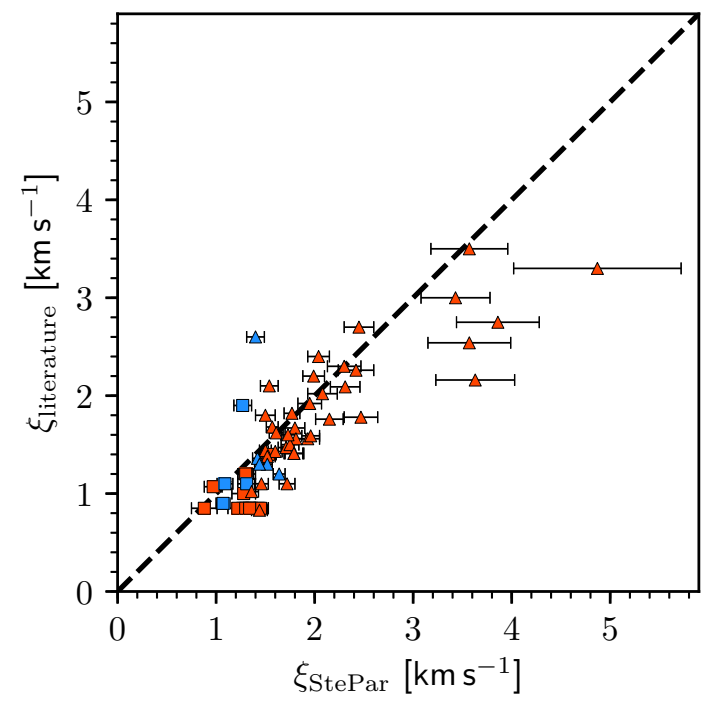

Figure 13. Micro-turbulent velocity derived for the sample with STEPAR, $\xi_{\text {StePar, }}$, versus literature values. Symbols are the same as in Fig. 1.

( $\Delta \log g>0.25$ dex). Among the outliers in $\log g$ are Arcturus and 7 Psc. According to Heiter et al. (2015), the $\log g$ value of Arcturus remains uncertain, with literature values ranging from 1.4 up to 2.0 dex, while both the $T_{\text {eff }}$ and $\log g$ values for the star 7 Psc are, in fact, not recommended for use as reference values. Among the outliers in $T_{\text {eff }}$ are the stars HD 49933, $\mu$ Leo, $\epsilon$ Vir, and 7 Psc. As stated by Heiter et al. (2015), the fundamental $T_{\text {eff }}$ value for the stars $\epsilon$ Vir and $\mu$ Leo is significantly lower ( $\sim 3$ per cent) than the value derived in spectroscopic studies. Lastly, at the hot regime, the typical spectroscopic $T_{\text {eff }}$ values computed for the star HD 49933 are generally larger.

Lastly, in Fig. 15, we show the final Fe I and Fe II abundances versus the excitation potential and the reduced $\mathrm{EW}$ of the lines, for the four reference CARMENES spectra (18 Sco, $\mu$ Cas, $\epsilon$ Vir, and Arcturus).

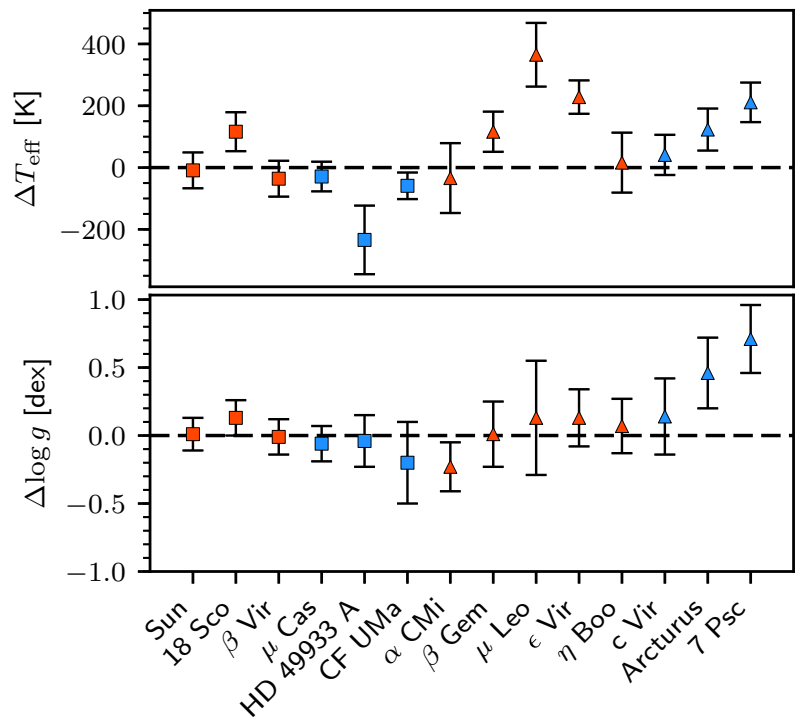

Figure 14. Differences in $T_{\text {eff }}$ and $\log g$ between this work and Heiter et al. (2015), with updated values from Jofré et al. (2018), for the Gaia benchmark stars in our sample. Symbols are the same as in Fig. 1.

\section{CONCLUSIONS}

In this work, we have expanded previous optical Fe I and Fe II line lists into the wavelength range covered by CARMENES, i.e. from 5300 to $17100 \AA$. The line lists are suited for FGK-type stars and relate to MRDs, MPDs, MRGs, and MPGs. For the first time, we provide Fe I and Fe II lines in the wavelength region between 6800 and $10000 \AA$. Altogether, these new line lists contain $653 \mathrm{Fe} I$ and $23 \mathrm{Fe}$ II lines, of which 351 and eight are new additions to the line lists compiled in Tabernero et al. (2019), respectively. This implies more than doubling the number of Fe I and Fe II lines useful for abundance and radial-velocity analyses. The availability of these $\mathrm{Fe} \mathrm{I}$ and $\mathrm{Fe}$ II line lists is also an asset for other new high-resolution near-infrared spectrographs such as SPIRou, GIANO, CRIRES+ , IRD, HPF, and NIRPS that also provide wavelength coverage in the near-infrared wavelength region.

We have reported that the star c Gem (HD 62285) is a new SB2 system, as shown by the cross-correlation with an atlas spectrum of Arcturus.

In addition, we have computed a homogenized set of stellar atmospheric parameters for a sample of 65 FGK-type stars observed with CARMENES by means of the EW method. We made a comprehensive comparison of our $T_{\text {eff }}, \log g$, and [Fe/ $\left.\mathrm{H}\right]$ values with those of virtually all relevant determinations of stellar atmospheric parameters of FGK-type stars. Our parameter determinations are in good agreement with the literature values in general, particularly with the region of the parameter space covered by the YaPSI isochrones (Spada et al. 2017) and the Gaia benchmark stars (Jofré et al. 2014, 2018; Heiter et al. 2015). The scarcity of Fe II lines in the NIR wavelength range covered by CARMENES prevented us from performing the stellar parameter determinations using this spectral region alone. However, when using both VIS and NIR CARMENES channel data, we found a broader $T_{\text {eff }}$ scale that seems to be linked to a higher sensitivity to effective temperature of the iron lines found in the NIR region.

The line selections provided in this work will be useful for the spectroscopic analysis of any FGK-type star simultaneously observed in the optical and near-infrared wavelength regions. Finally, 

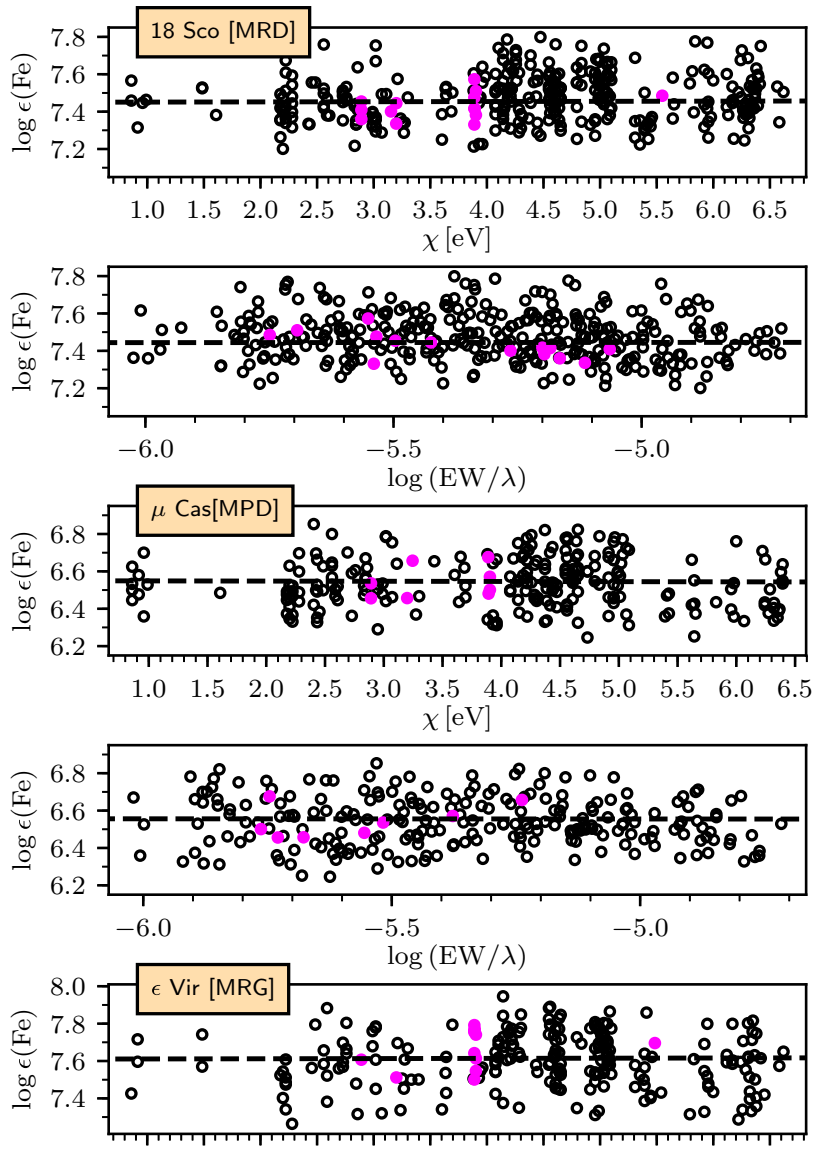

$\begin{array}{llllllllllll}1.0 & 1.5 & 2.0 & 2.5 & 3.0 & 3.5 & 4.0 & 4.5 & 5.0 & 5.5 & 6.0 & 6.5\end{array}$ $\chi[\mathrm{eV}]$
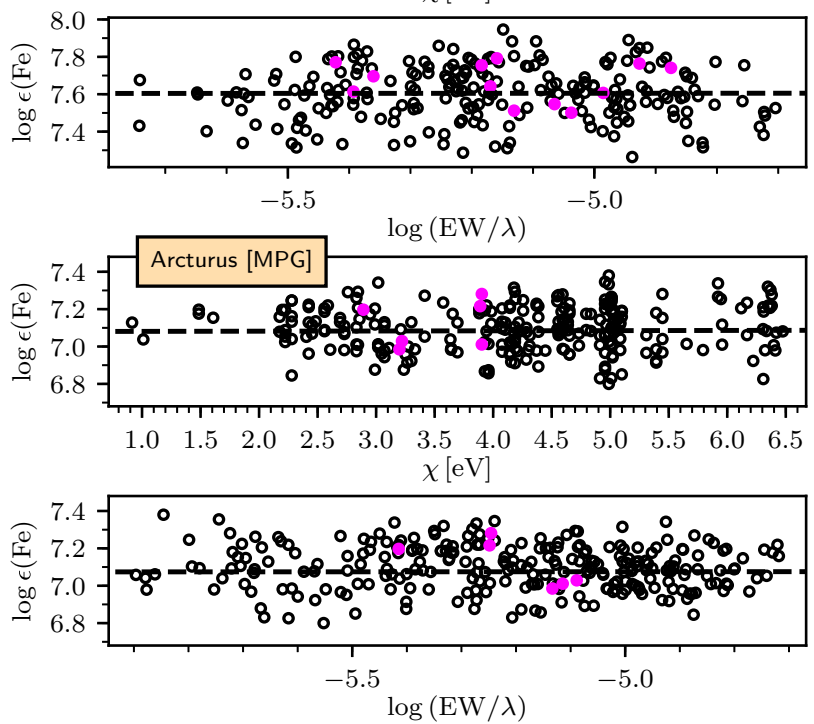

Figure 15. From top to bottom: line iron abundance retrieved by STEPAR for the final solution of the four reference stars: 18 Sco, $\mu$ Cas, $\epsilon$ Vir, and Arcturus. $\log \epsilon(\mathrm{Fe} \mathrm{I})$ stands for the Fe abundance returned by the Fe lines, while $\log (\mathrm{EW} / \lambda)$ is their reduced EWs. The open black dots represent Fe I lines, whereas the pink dots are Fe II lines. The dashed black lines represent the least-squares fit to the data points. in a forthcoming publication we plan to expand optical line lists of additional chemical species into the NIR covered by CARMENES and thus assess the impact of the near-infrared wavelength region upon chemical abundance computations for FGK-type stars.

\section{ACKNOWLEDGEMENTS}

CARMENES is an instrument for the Centro Astronómico Hispano en Andalucía at Calar Alto (CAHA). CARMENES is funded by the German Max-Plank Gesellschaft (MPG), the Spanish Consejo Superior de Investigaciones Científicas (CSIC), the European Union through FEDER/ERF FICTS-2011-02 funds, and the members of the CARMENES Consortium (Max-Plank-Institut für Astronomie, Instituto de Astrofísica de Andalucía, Landessternwarte Königstuhl, Institut de Ciències de l'Espai, Institut für Astrophysik Göttingen, Universidad Complutense de Madrid, Thüringer Landessternwarte Tautenberg, Instituto de Astrofísica de Canarias, Hamburger Sternwarte, Centro de Astrobiología and Centro Astronómico Hispano en Andalucía), with additional contributions by the Ministerio de Asuntos Económicos y Transformación Digital, the German Research Foundation (DFG) through the Major Research Instrumentation Programme and DFG Research Unit FOR2544 'Blue Planets around Red Stars', the Klaus Tschira Stiftung, the states of Baden-Württemberg and Niedersachsen, and by the Junta de Andalucía. The authors acknowledge financial support from the Fundação para a Ciência e a Tecnologia (FCT) through national funds (PTDC/FIS-AST/28953/2017) and by Fundo Europeu de Desenvolvimento Regional (FEDER) through COMPETE2020 - Programa Operacional Competitividade e Internacionalização (POCI01-0145-FEDER-028953), the Ministerio de Ciencia e Innovación through fellowship FPU15/01476, and projects AYA2016-79425C3-1/2/3-P, and the Universidad Complutense de Madrid. JIGH acknowledges financial support from the Ministerio de Ciencia e Innovación under the 2013 Ramón y Cajal programme RYC-201314875, and from the project AYA2017-86389-P. This work has made use of the VALD database, operated at Uppsala University, the Institute of Astronomy RAS in Moscow, and the University of Vienna. We thank Calar Alto Observatory for the allocation of director's discretionary time to this programme. EM would also like to warmly thank the staff at the Hamburger Sternwarte for their hospitality during his stay funded by project EST18/00162 from the Ministerio de Ciencia e Innovación. Based on data from the CARMENES data archive at CAB (INTA-CSIC).

\section{REFERENCES}

Adibekyan V. Z., González Hernández J. I., Delgado Mena E., Sousa S. G., Santos N. C., Israelian G., Figueira P., Bertran de Lis S., 2014, A\&A, 564, L15

Allende Prieto C., Barklem P. S., Lambert D. L., Cunha K., 2004, A\&A, 420, 183

Allende Prieto C. et al., 2008, Astron. Nachr., 329, 1018

Andreasen D. T., Sousa S. G., Delgado Mena E., Santos N. C., Tsantaki M., Rojas-Ayala B., Neves V., 2016, A\&A, 585, A143

Artigau É. et al., 2014, in Ramsay S. K., McLean I. S., Takami H., eds, Proceedings SPIE Conference Series, Vol. 9147, Ground-based and Airborne Instrumentation for Astronomy V, Proceedings SPIE Conference Series, Bellingham, p. 914715

Aurière M., 2003, in Arnaud J., Meunier N., eds, EAS Publ. Ser. Vol. 9 Magnetism and Activity of the Sun and Stars. Cambridge Univ. Press, Cambridge, p. 105

Bensby T., Feltzing S., Oey M. S., 2014, A\&A, 562, A71

Blanco-Cuaresma S., 2019, MNRAS, 486, 2075 
Blanco-Cuaresma S., Soubiran C., Heiter U., Jofré P., 2014, Astrophysics Source Code Library, record ascl:1409.006

Caballero J. A. et al., 2016, in Peck A. B., Seaman R. L., Benn C. R., eds, Proc. SPIE Conf. Ser. Vol. 9910, Observatory Operations: Strategies, Processes, and Systems VI. SPIE, Bellingham, p. 99100E

Clough S. A., Shephard M. W., Mlawer E. J., Delamere J. S., Iacono M. J., Cady-Pereira K., Boukabara S., Brown P. D., 2005, J. Quant. Spectrosc. Radiat. Transfer, 91, 233

da Silva L. et al., 2006, A\&A, 458, 609

da Silva R., Milone A. d. C., Rocha-Pinto H. J., 2015, A\&A, 580, A24

De Medeiros J. R., do Nascimento J. D. J., Sankarankutty S., Costa J. M., Maia M. R. G., 2000, A\&A, 363, 239

De Medeiros J. R., Udry S., Burki G., Mayor M., 2002, A\&A, 395, 97

De Silva G. M. et al., 2015, MNRAS, 449, 2604

Delgado Mena E. et al., 2018, A\&A, 619, A2

dos Santos L. A. et al., 2016, A\&A, 592, A156

Famaey B., Jorissen A., Luri X., Mayor M., Udry S., Dejonghe H., Turon C., 2005, A\&A, 430, 165

Gaia Collaboration, 2018, A\&A, 616, A1

Gilmore G. et al., 2012, The Messenger, 147, 25

Girard T. M. et al., 2000, AJ, 119, 2428

Gontcharov G. A., 2006, Astron. Lett., 32, 759

Gordon I. E., Rothman L. S., Tan Y., Kochanov R. V., Hill C., 2017, 72nd International Symposium on Molecular Spectroscopy, p. TJ08

Gray D. F., 2018, ApJ, 869, 81

Gray D. F., Toner C. G., 1986, ApJ, 310, 277

Gustafsson B., Edvardsson B., Eriksson K., Jørgensen U. G., Nordlund Å., Plez B., 2008, A\&A, 486, 951

Hatzes A., CRIRES + Team, 2017, American Astronomical Society Meeting Abstracts \#230, p. 117.02

Hayes C. R. et al., 2018, ApJ, 852, 49

Heiter U., Luck R. E., 2003, AJ, 126, 2015

Heiter U., Jofré P., Gustafsson B., Korn A. J., Soubiran C., Thévenin F., 2015, A\&A, 582, A49

Hekker S., Meléndez J., 2007, A\&A, 475, 1003

Hinkle K., Wallace L., Valenti J., Harmer D., 2000, Visible and Near Infrared Atlas of the Arcturus Spectrum 3727-9300 A, Astronomical Society of the Pacific, San Francisco

Jofré P. et al., 2014, A\&A, 564, A133

Jofré E., Petrucci R., Saffe C., Saker L., de la Villarmois E. A., Chavero C., Gómez M., Mauas P. J. D., 2015, A\&A, 574, A50

Jofré P., Heiter U., Tucci Maia M., Soubiran C., Worley C. C., Hawkins K., Blanco-Cuaresma S., Rodrigo C., 2018, Res. Notes Am. Astron. Soc., 2,152

Jofré P., Heiter U., Soubiran C., 2019, ARA\&A, 57, 5

Karataş Y., Bilir S., Eker Z., Demircan O., 2004, MNRAS, 349, 1069

Kasting J. F., Whitmire D. P., Reynolds R. T., 1993, Icarus, 101, 108

Kausch W. et al., 2015, A\&A, 576, A78

Keenan P. C., McNeil R. C., 1989, ApJS, 71, 245

Kopparapu R. K. et al., 2013, ApJ, 765, 131

Kotani T. et al., 2014, in Ramsay S. K., McLean I. S., Takami H., eds, Proc. SPIE Conf. Ser. Vol. 9147, Ground-based and Airborne Instrumentation for Astronomy V. SPIE, Bellingham, p. 914714

Kupka F., Piskunov N., Ryabchikova T. A., Stempels H. C., Weiss W. W., 1999, A\&AS, 138, 119

Kupka F. G., Ryabchikova T. A., Piskunov N. E., Stempels H. C., Weiss W. W., 2000, Balt. Astron., 9, 590

Lèbre A., de Laverny P., Do Nascimento J. D. J., de Medeiros J. R., 2006, A\&A, 450, 1173

Lebzelter T. et al., 2012, A\&A, 539, A109

Liu Y. J., Zhao G., Shi J. R., Pietrzyński G., Gieren W., 2007, MNRAS, 382, 553

Luck R. E., 2014, AJ, 147, 137

Lyubimkov L. S., Lambert D. L., Rostopchin S. I., Rachkovskaya T. M., Poklad D. B., 2010, MNRAS, 402, 1369

Lyubimkov L. S., Lambert D. L., Kaminsky B. M., Pavlenko Y. V., Poklad D. B., Rachkovskaya T. M., 2012, MNRAS, 427, 11
Majewski S. R. et al., 2017, AJ, 154, 94

Maldonado J., Martínez-Arnáiz R. M., Eiroa C., Montes D., Montesinos B., 2010, A\&A, 521, A12

Mann A. W. et al., 2019, ApJ, 871, 63

Martínez-Arnáiz R., Maldonado J., Montes D., Eiroa C., Montesinos B., 2010, A\&A, 520, A79

Massarotti A., Latham D. W., Stefanik R. P., Fogel J., 2008, AJ, 135, 209

Mayor M. et al., 2003, The Messenger, 114, 20

McCarthy K., Wilhelm R. J., 2014, AJ, 148, 70

McWilliam A., 1990, ApJS, 74, 1075

Meléndez J., Barbuy B., 2009, A\&A, 497, 611

Montes D. et al., 2018, MNRAS, 479, 1332

Morel T. et al., 2014, A\&A, 564, A119

Morton D. C., 2000, ApJS, 130, 403

Mucciarelli A., Pancino E., Lovisi L., Ferraro F. R., Lapenna E., 2013, ApJ, 766,78

Nicholls C. P. et al., 2017, A\&A, 598, A79

Nidever D. L., Marcy G. W., Butler R. P., Fischer D. A., Vogt S. S., 2002, ApJS, 141, 503

Oliva E., Sanna N., Rainer M., Massi F., Tozzi A., Origlia L., 2018, in Evans C. J., Simard L., Takami H., eds, Proc. SPIE Conf. Ser. Vol. 7015, Ground-based and Airborne Instrumentation for Astronomy VII. SPIE, Bellingham, p. 1070274

Origlia L. et al., 2014, in Ramsay S. K., McLean I. S., Takami H., eds, Proc. SPIE Conf. Ser. Vol. 9147, Ground-based and Airborne Instrumentation for Astronomy V. SPIE, Bellingham, p. 91471E

Park S. et al., 2018, ApJS, 238, 29

Passegger V. M. et al., 2019, A\&A, 627, A161

Pavlenko Y. V., Jenkins J. S., Jones H. R. A., Ivanyuk O., Pinfield D. J., 2012, MNRAS, 422, 542

Piskunov N. E., Valenti J. A., 2002, A\&A, 385, 1095

Piskunov N., Valenti J. A., 2017, A\&A, 597, A16

Piskunov N. E., Kupka F., Ryabchikova T. A., Weiss W. W., Jeffery C. S., 1995, A\&AS, 112, 525

Pourbaix D. et al., 2004, A\&A, 424, 727

Press W. H., Teukolsky S. A., Vetterling W. T., Flannery B. P., 2002, Numerical Recipes in $\mathrm{C}++$ : The Art of Scientific Computing. Cambridge Univ. Press, Cambridge

Quirrenbach A. et al., 2018, in Evans C. J., Simard L., Takami H., eds, Proc. SPIE Conf. Ser. Vol. 10702, Ground-based and Airborne Instrumentation for Astronomy VII. SPIE, Bellingham, p. 107020W

Reiners A. et al., 2018, A\&A, 612, A49

Rodrigues T. S. et al., 2014, MNRAS, 445, 2758

Rodrigues T. S. et al., 2017, MNRAS, 467, 1433

Ryabchikova T., Piskunov N., Kurucz R. L., Stempels H. C., Heiter U., Pakhomov Y., Barklem P. S., 2015, Phys. Scr., 90, 054005

Santos N. C. et al., 2013, A\&A, 556, A150

Scarfe C. D., Funakawa H., Delaney P. A., Barlow D. J., 1983, J. R. Astron. Soc. Can., 77, 126

Schweitzer A. et al., 2019, A\&A, 625, A68

Skrutskie M. F. et al., 2006, AJ, 131, 1163

Smette A. et al., 2015, A\&A, 576, A77

Sneden C. A., 1973, PhD thesis, The University of Texas at Austin

Soubiran C., Jasniewicz G., Chemin L., Crifo F., Udry S., Hestroffer D., Katz D., 2013, A\&A, 552, A64

Soubiran C., Le Campion J.-F., Brouillet N., Chemin L., 2016, A\&A, 591 , A118

Sousa S. G., Santos N. C., Israelian G., Mayor M., Monteiro M. J. P. F. G., 2007, A\&A, 469, 783

Sousa S. G. et al., 2008, A\&A, 487, 373

Spada F., Demarque P., Kim Y.-C., Boyajian T. S., Brewer J. M., 2017, ApJ, 838,161

Spencer Jones H., Furner H. H., 1937, MNRAS, 98, 92

Steinmetz M. et al., 2006, AJ, 132, 1645

Tabernero H. M., Montes D., González Hernández J. I., 2012, A\&A, 547, A13

Tabernero H. M., Montes D., González Hernández J. I., Ammler-von Eiff M., 2017, A\&A, 597, A33 
Tabernero H. M., Dorda R., Negueruela I., González-Fernández C., 2018, MNRAS, 476, 3106

Tabernero H. M., Marfil E., Montes D., González Hernández J. I., 2019, A\&A, 628, A131

Takeda Y. et al., 2005, PASJ, 57, 13

Takeda Y., Sato B., Murata D., 2008, PASJ, 60, 781

Thygesen A. O. et al., 2012, A\&A, 543, A160

Tsantaki M., Sousa S. G., Adibekyan V. Z., Santos N. C., Mortier A., Israelian G., 2013, A\&A, 555, A150

Valenti J. A., Fischer D. A., 2005, ApJS, 159, 141

van Leeuwen F., 2007, A\&A, 474, 653

Wildi F. et al., 2017, Shaklan S., ed., Proc. SPIE Conf. Ser. Vol. 10400, Techniques and Instrumentation for Detection of Exoplanets VIII. SPIE, Bellingham, p. 1040018

Worek T. F., Beardsley W. R., 1977, ApJ, 217, 134

Wright J. T. et al., 2018, American Astronomical Society Meeting Abstracts \#231, p. 246.45

Wu Y., Singh H. P., Prugniel P., Gupta R., Koleva M., 2011, A\&A, 525, A71

Zamora O. et al., 2015, AJ, 149, 181

Zechmeister M., Anglada-Escudé G., Reiners A., 2014, A\&A, 561, A59

\section{SUPPORTING INFORMATION}

Supplementary data are available at MNRAS online.

Fig. A1. CARMENES spectrum of 18 Sco.
Table A2. Stellar atmospheric parameters of the selected sample under STEPAR and $\log g$ values obtained with PARAM assuming parallaxes from Gaia DR2 and the Hipparcos mission.

Table A3. Stellar atmospheric parameters of the selected sample under STEPAR restricted to the optical and $\log g$ values obtained with PARAM assuming parallaxes from Gaia DR2 and the Hipparcos mission.

Please note: Oxford University Press is not responsible for the content or functionality of any supporting materials supplied by the authors. Any queries (other than missing material) should be directed to the corresponding author for the article.

\section{APPENDIX A: APPENDIX}

In Table A1, we give the literature values of the stellar atmospheric parameters for the selected sample. In Tables A2 and A3, we give the stellar atmospheric parameters computed with STEPAR in the whole VIS + NIR region and VIS region, respectively. In Tables A4 and A5, we list the Fe I and Fe II lines along with their parameters, respectively, for MRDs, MPDs, MRGs, and MPGs. Finally, we include the CARMENES spectrum of the reference, MRD 18 Sco in Fig. A6, along with the Fe I and Fe II lines indicated in red and green, respectively. 


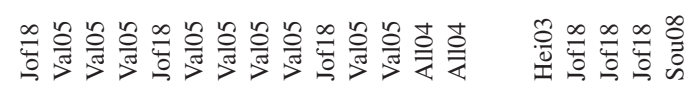

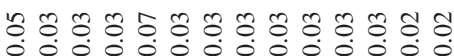

$\mathrm{H}+\mathrm{H} H+\mathrm{H} H \mathrm{H}+\mathrm{H} H \mathrm{H}+\mathrm{H} H \mathrm{H}$

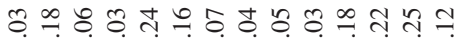

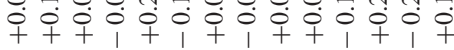
$\stackrel{\circ}{\circ}$

$\stackrel{8}{\circ} 1$

กิ

$\mathrm{H}+\mathrm{H}+\mathrm{H}+\mathrm{H}+\mathrm{H}+\mathrm{H}+\mathrm{H}+\mathrm{H}+\mathrm{H}+\mathrm{H}+$ तิ

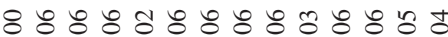

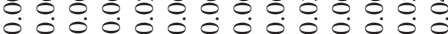

$H+H+H+H+H+H+H+H+H+H$

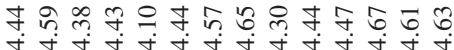

一专抳扵找拐拐

$\mathrm{H}+\mathrm{H}+\mathrm{H} H \mathrm{H}+\mathrm{H} H \mathrm{H}+\mathrm{H}+\mathrm{H} H$

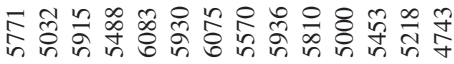

느넝ㅇㅇㅇㅇㅇㅇㅇㅇ으응

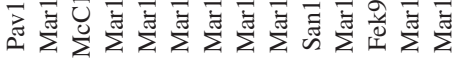

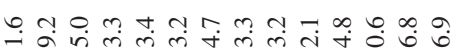

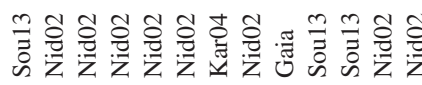

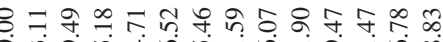

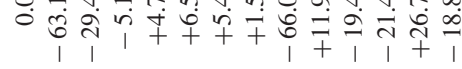

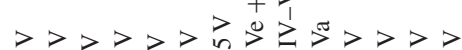

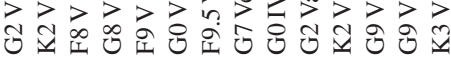

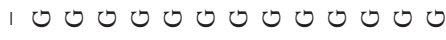

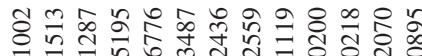
०००0. H H H H H H H H H H H H H

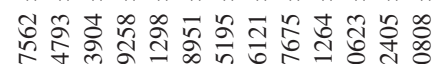

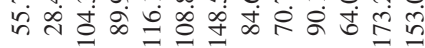

m n चำ

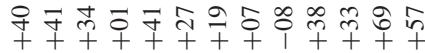

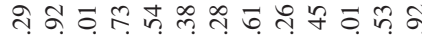

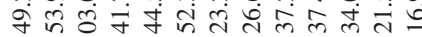

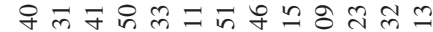

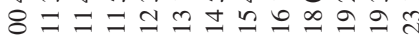

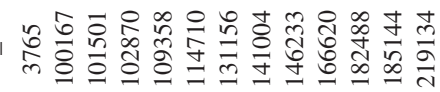

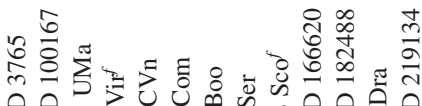

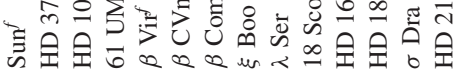

mీ

i 100

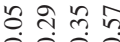

$\mathrm{H}+\mathrm{H} H$

웅우

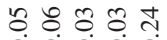

$\mathrm{H} H \mathrm{H} H \mathrm{H}$

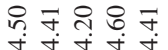

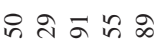

H H H H H

유윯유용

은은은은

范芯离志志

ஸุ ๆ ํํำ 完

ôd

으의

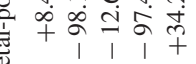

$+\geq>z^{2}>$

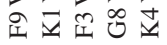

0 ○ 0

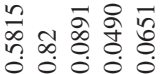
$H+H+H+H$

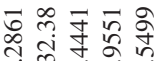

픙

我 $\infty 00.0$

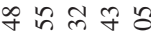

命

สิ่ล 守的守的

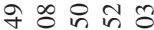
$8 \div 8=\Sigma$

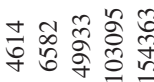

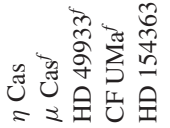

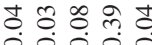

$\mathrm{H} H \mathrm{H} H+\mathrm{H}$

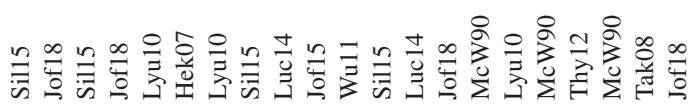

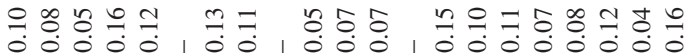

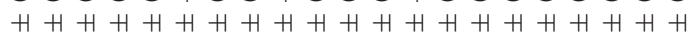

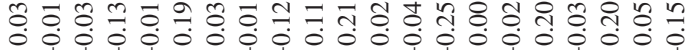
$1+1+1$
0
0

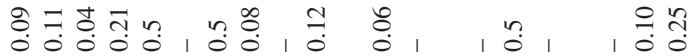
$\mathrm{H}+\mathrm{H} H \mathrm{H}+\mathrm{H}+\mathrm{H} H \mathrm{H}, \mathrm{H}, \mathrm{H}+\mathrm{H}, \mathrm{H}+\mathrm{H}$

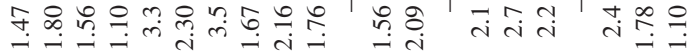

ஸి $H+H+H+H+H+H+H+H H+H+H+H+H+H$

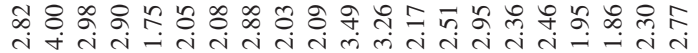

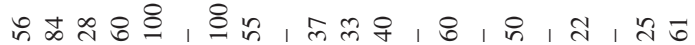
$H+H+H+H H H H+H H H+H+H H H H+H$

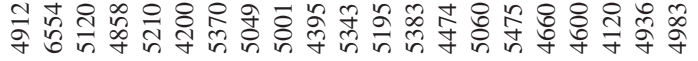

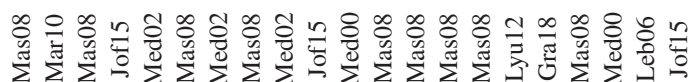

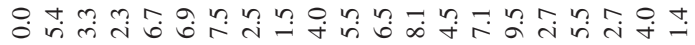
崽

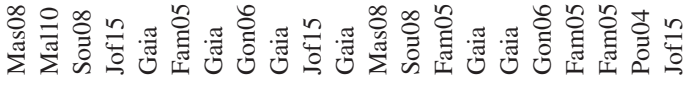

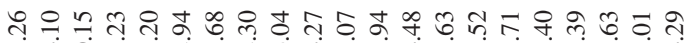

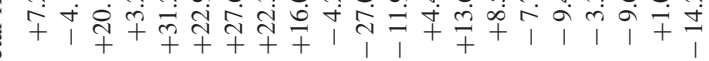

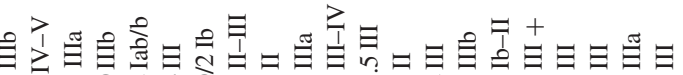

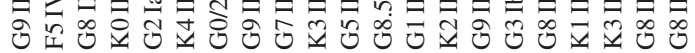

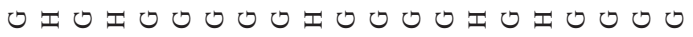

तิ $H+H+H+H+H+H H+H+H+H+H+H+H+H$

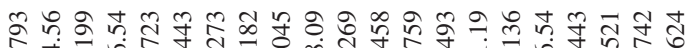

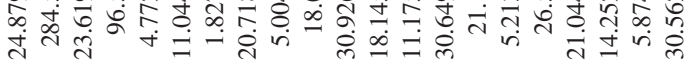

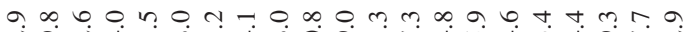
तं तें

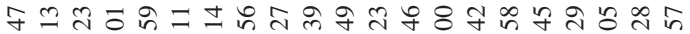

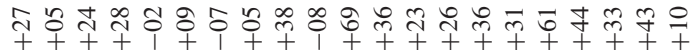

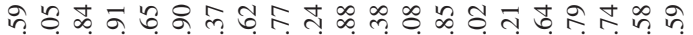
ๆ ฟै ㄷㅇㅇㅇㅇㅇㅇㅇㅇㅇㅇㅇㅇㅇ을

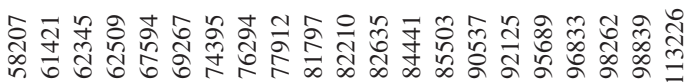

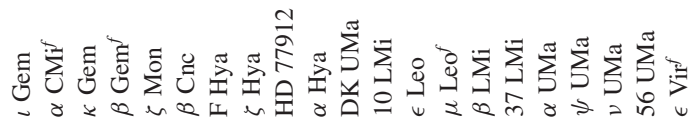




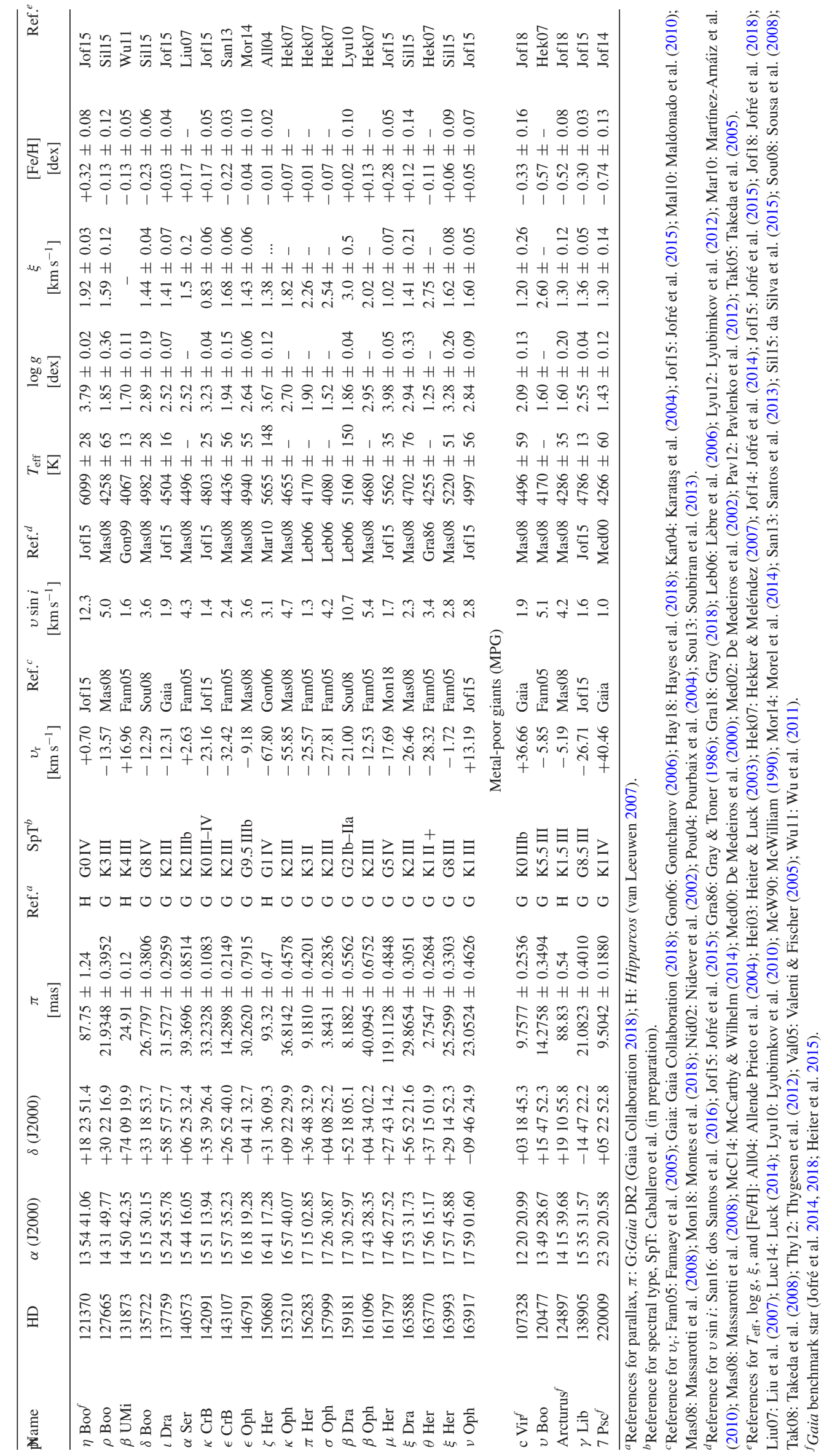


Table A2. Stellar atmospheric parameters of the selected sample under STEPAR and $\log g$ values obtained with PARAM assuming parallaxes from Gaia DR2 and the Hipparcos mission.

\begin{tabular}{lccccccr}
\hline Name & $\begin{array}{c}v_{\mathrm{r}} \\
{\left[\mathrm{km} \mathrm{s}^{-1}\right]}\end{array}$ & $\mathrm{S} / \mathrm{N}$ & $\begin{array}{c}T_{\text {eff }} \\
{[\mathrm{K}]}\end{array}$ & $\begin{array}{c}\log g \\
{[\mathrm{dex}]}\end{array}$ & $\begin{array}{c}\log g_{\text {PARAM }} \\
{[\mathrm{dex}]}\end{array}$ & $\begin{array}{c}\xi \\
{\left[\mathrm{km} \mathrm{s}^{-1}\right]}\end{array}$ & $\begin{array}{c}{[\mathrm{Fe} / \mathrm{H}]} \\
{[\mathrm{dex}]}\end{array}$ \\
\hline \multicolumn{7}{c}{ Metal-rich dwarfs $(\mathrm{MRD})$} \\
Sun & $0.00 \pm 0.00$ & 249 & $5768 \pm 58$ & $4.45 \pm 0.12$ & - & $1.31 \pm 0.09$ & $-0.01 \pm 0.04$ \\
HD 3765 & $-63.18 \pm 0.50$ & 138 & $5310 \pm 81$ & $4.63 \pm 0.24$ & $4.55 \pm 0.02$ & $1.43 \pm 0.10$ & $+0.12 \pm 0.03$ \\
HD 100167 & $-29.41 \pm 0.52$ & 98 & $5898 \pm 57$ & $4.47 \pm 0.12$ & $4.43 \pm 0.02$ & $1.27 \pm 0.09$ & $+0.01 \pm 0.04$ \\
61 UMa & $-5.47 \pm 0.46$ & 248 & $5555 \pm 59$ & $4.59 \pm 0.13$ & $4.52 \pm 0.02$ & $1.36 \pm 0.10$ & $-0.07 \pm 0.03$ \\
$\beta$ Vir & $+4.58 \pm 0.65$ & 345 & $6199 \pm 63$ & $4.23 \pm 0.13$ & $4.09 \pm 0.02$ & $1.53 \pm 0.08$ & $+0.17 \pm 0.04$ \\
$\beta$ CVn & $+6.34 \pm 0.49$ & 241 & $5902 \pm 61$ & $4.41 \pm 0.13$ & $4.37 \pm 0.03$ & $1.22 \pm 0.10$ & $-0.20 \pm 0.04$ \\
$\beta$ Com & $+5.41 \pm 0.57$ & 198 & $6000 \pm 58$ & $4.44 \pm 0.12$ & $4.42 \pm 0.01$ & $1.30 \pm 0.08$ & $+0.04 \pm 0.04$ \\
$\xi$ Boo & $+1.81 \pm 0.40$ & 67 & $5403 \pm 52$ & $4.48 \pm 0.12$ & $4.54 \pm 0.02$ & $1.45 \pm 0.08$ & $-0.19 \pm 0.03$ \\
$\lambda$ Ser & $-66.22 \pm 0.60$ & 109 & $5835 \pm 54$ & $4.00 \pm 0.14$ & $4.22 \pm 0.02$ & $1.34 \pm 0.07$ & $-0.04 \pm 0.04$ \\
18 Sco & $+11.93 \pm 0.59$ & 298 & $5774 \pm 58$ & $4.43 \pm 0.13$ & $4.41 \pm 0.04$ & $1.30 \pm 0.08$ & $0.00 \pm 0.04$ \\
\hline
\end{tabular}

Table A3. Stellar atmospheric parameters of the selected sample under STEPAR restricted to the optical and $\log g$ values obtained with PARAM assuming parallaxes from Gaia DR2 and the Hipparcos mission.

\begin{tabular}{lccccccc}
\hline Name & $\begin{array}{c}v_{\mathrm{r}} \\
{\left[\mathrm{km} \mathrm{s}^{-1}\right]}\end{array}$ & $\mathrm{S} / \mathrm{N}$ & $\begin{array}{c}T_{\text {eff }} \\
{[\mathrm{K}]}\end{array}$ & $\begin{array}{c}\log g \\
{[\mathrm{dex}]}\end{array}$ & $\begin{array}{c}\log g_{\text {PARAM }} \\
{[\mathrm{dex}]}\end{array}$ & $\begin{array}{c}\xi \\
{\left[\mathrm{km} \mathrm{s}^{-1}\right]}\end{array}$ & $\begin{array}{c}{[\mathrm{Fe} / \mathrm{H}]} \\
{[\mathrm{dex}]}\end{array}$ \\
\hline \multicolumn{7}{c}{ Metal-rich dwarfs (MRD) } \\
Sun & $0.00 \pm 0.00$ & 249 & $5787 \pm 54$ & $4.42 \pm 0.11$ & - & $0.98 \pm 0.08$ & $+0.02 \pm 0.03$ \\
HD 3765 & $-63.18 \pm 0.50$ & 138 & $5206 \pm 84$ & $4.62 \pm 0.22$ & $4.55 \pm 0.02$ & $1.17 \pm 0.12$ & $+0.16 \pm 0.04$ \\
HD 100167 & $-29.41 \pm 0.52$ & 98 & $5942 \pm 54$ & $4.49 \pm 0.10$ & $4.43 \pm 0.02$ & $1.04 \pm 0.09$ & $+0.04 \pm 0.04$ \\
61 UMa & $-5.47 \pm 0.46$ & 248 & $5576 \pm 56$ & $4.56 \pm 0.12$ & $4.52 \pm 0.02$ & $1.07 \pm 0.09$ & $-0.01 \pm 0.03$ \\
$\beta$ Vir & $+4.58 \pm 0.65$ & 345 & $6255 \pm 64$ & $4.20 \pm 0.12$ & $4.09 \pm 0.02$ & $1.45 \pm 0.08$ & $+0.23 \pm 0.04$ \\
$\beta$ CVn & $+6.34 \pm 0.49$ & 241 & $5967 \pm 57$ & $4.40 \pm 0.12$ & $4.37 \pm 0.03$ & $0.96 \pm 0.09$ & $-0.14 \pm 0.04$ \\
$\beta$ Com & $+5.41 \pm 0.57$ & 198 & $6105 \pm 56$ & $4.42 \pm 0.11$ & $4.42 \pm 0.01$ & $1.18 \pm 0.07$ & $+0.02 \pm 0.04$ \\
$\xi$ Boo & $+1.81 \pm 0.40$ & 67 & $5536 \pm 55$ & $4.50 \pm 0.12$ & $4.54 \pm 0.02$ & $1.25 \pm 0.09$ & $-0.15 \pm 0.03$ \\
$\lambda$ Ser & $-66.22 \pm 0.60$ & 109 & $5950 \pm 56$ & $4.31 \pm 0.14$ & $4.22 \pm 0.02$ & $1.22 \pm 0.08$ & $+0.05 \pm 0.04$ \\
18 Sco & $+11.93 \pm 0.59$ & 298 & $5786 \pm 54$ & $4.40 \pm 0.10$ & $4.41 \pm 0.04$ & $0.99 \pm 0.09$ & $+0.02 \pm 0.04$ \\
\hline
\end{tabular}


Table A4. Merged Fe I line lists.

\begin{tabular}{|c|c|c|c|c|c|c|c|}
\hline \multirow{2}{*}{$\begin{array}{l}\lambda_{\text {air }} \\
{[\AA]}\end{array}$} & \multirow{2}{*}{$\begin{array}{c}\chi_{\mathrm{I}} \\
{[\mathrm{eV}]}\end{array}$} & \multirow[t]{2}{*}{$\log g f$} & \multicolumn{4}{|c|}{ Line list $^{a}$} & \multirow[t]{2}{*}{ Reference $^{b}$} \\
\hline & & & MRD & MPD & MRG & MPG & \\
\hline 5307.361 & 1.61 & -2.912 & • & $\bullet$ & & - & Sou08 \\
\hline 5321.108 & 4.44 & -1.089 & & & & - & Sou08 \\
\hline 5322.041 & 2.28 & -2.802 & - & & & - & Sou08 \\
\hline 5339.929 & 3.27 & -0.635 & & & & - & Sou08 \\
\hline 5364.871 & 4.45 & +0.228 & & & $\bullet$ & $\bullet$ & Sou08 \\
\hline 5373.709 & 4.47 & -0.710 & - & - & & & Sou08 \\
\hline 5379.574 & 3.70 & -1.514 & - & - & $\bullet$ & - & Sou08 \\
\hline 5386.333 & 4.15 & -1.670 & $\bullet$ & & $\bullet$ & $\bullet$ & Sou08 \\
\hline 5389.479 & 4.42 & -0.410 & & - & $\bullet$ & & Sou08 \\
\hline 5397.618 & 3.63 & -2.528 & & & $\bullet$ & & Sou08 \\
\hline 5398.279 & 4.45 & -0.630 & $\bullet$ & $\bullet$ & $\bullet$ & & Sou08 \\
\hline 5400.501 & 4.37 & -0.160 & - & - & & & Sou08 \\
\hline 5401.266 & 4.32 & -1.820 & - & & - & & Sou08 \\
\hline 5409.133 & 4.37 & -1.200 & - & $\bullet$ & & & Sou08 \\
\hline 5417.033 & 4.42 & -1.580 & $\bullet$ & & $\bullet$ & & Sou08 \\
\hline 5424.068 & 4.32 & +0.520 & & - & & & Sou08 \\
\hline 5436.295 & 4.39 & -1.440 & - & & - & - & Sou08 \\
\hline 5436.588 & 2.28 & -2.964 & & & $\bullet$ & & Sou08 \\
\hline 5441.339 & 4.31 & -1.630 & - & & $\bullet$ & - & Sou08 \\
\hline 5445.042 & 4.39 & -0.020 & & $\bullet$ & - & - & Sou08 \\
\hline 5460.873 & 3.07 & -3.426 & & & $\bullet$ & $\bullet$ & Sou08 \\
\hline 5461.550 & 4.45 & -1.800 & - & & $\bullet$ & & Sou08 \\
\hline 5463.275 & 4.44 & +0.070 & & & $\bullet$ & - & Sou08 \\
\hline 5464.280 & 4.14 & -1.402 & & & $\bullet$ & & Sou08 \\
\hline 5466.396 & 4.37 & -0.630 & $\bullet$ & $\bullet$ & & $\bullet$ & Sou08 \\
\hline 5470.093 & 4.45 & -1.710 & $\bullet$ & & $\bullet$ & & Sou08 \\
\hline 5472.709 & 4.21 & -1.495 & - & & & & Sou08 \\
\hline 5473.900 & 4.15 & -0.720 & $\bullet$ & $\bullet$ & & & Sou08 \\
\hline 5483.099 & 4.15 & -1.392 & - & & & - & Sou08 \\
\hline 5501.465 & 0.96 & -3.047 & & - & & - & Sou08 \\
\hline 5506.778 & 0.99 & -2.797 & & $\bullet$ & & & Sou08 \\
\hline 5522.446 & 4.21 & -1.550 & $\bullet$ & $\bullet$ & & & Sou08 \\
\hline 5536.580 & 2.83 & -3.810 & & & & $\bullet$ & Sou08 \\
\hline 5539.280 & 3.64 & -2.660 & & & & $\bullet$ & Sou08 \\
\hline 5543.147 & 3.69 & -1.570 & & $\bullet$ & & & Sou08 \\
\hline 5543.935 & 4.22 & -1.140 & - & - & - & - & Sou08 \\
\hline 5546.505 & 4.37 & -1.310 & & - & & & Sou08 \\
\hline 5549.949 & 3.69 & -2.910 & & & $\bullet$ & & Sou08 \\
\hline 5554.894 & 4.55 & -0.440 & & $\bullet$ & & & Sou08 \\
\hline 5560.211 & 4.43 & -1.190 & $\bullet$ & $\bullet$ & - & & Sou08 \\
\hline 5572.842 & 3.40 & -0.275 & & & & - & Sou08 \\
\hline 5576.089 & 3.43 & -1.000 & & $\bullet$ & $\bullet$ & - & Sou08 \\
\hline 5618.632 & 4.21 & -1.276 & $\bullet$ & - & - & - & Sou08 \\
\hline 5619.595 & 4.39 & -1.700 & $\bullet$ & & & $\bullet$ & Sou08 \\
\hline 5633.946 & 4.99 & -0.270 & & $\bullet$ & & & Sou08 \\
\hline 5635.822 & 4.26 & -1.890 & - & & & & Sou08 \\
\hline 5636.695 & 3.64 & -2.610 & $\bullet$ & & $\bullet$ & & Sou08 \\
\hline 5638.262 & 4.22 & -0.870 & $\bullet$ & $\bullet$ & & & Sou08 \\
\hline 5641.434 & 4.26 & -1.180 & $\bullet$ & - & & & Sou08 \\
\hline 5649.987 & 5.10 & -0.920 & • & & & - & Sou08 \\
\hline 5651.468 & 4.47 & -2.000 & $\bullet$ & & & & Sou08 \\
\hline 5652.317 & 4.26 & -1.950 & $\bullet$ & & & & Sou08 \\
\hline 5653.864 & 4.39 & -1.640 & $\bullet$ & & & $\bullet$ & Sou08 \\
\hline 5655.176 & 5.06 & -0.640 & $\bullet$ & & & - & Sou08 \\
\hline 5661.344 & 4.28 & -1.736 & $\bullet$ & & & - & Sou08 \\
\hline 5662.516 & 4.18 & -0.573 & - & $\bullet$ & & - & Sou08 \\
\hline 5679.023 & 4.65 & -0.920 & $\bullet$ & $\bullet$ & $\bullet$ & $\bullet$ & Sou08 \\
\hline 5691.496 & 4.30 & -1.520 & & $\bullet$ & & & Sou08 \\
\hline 5696.088 & 4.55 & -1.720 & $\bullet$ & & & & Sou08 \\
\hline 5701.543 & 2.56 & -2.216 & $\bullet$ & $\bullet$ & & & Sou08 \\
\hline 5705.464 & 4.30 & -1.355 & $\bullet$ & & & & Sou08 \\
\hline 5717.832 & 4.28 & -1.130 & $\bullet$ & - & $\bullet$ & - & Sou08 \\
\hline 5720.886 & 4.55 & -1.950 & - & & • & - & Sou08 \\
\hline
\end{tabular}


Table A4 - continued

\begin{tabular}{|c|c|c|c|c|c|c|c|}
\hline \multirow{2}{*}{$\begin{array}{l}\lambda_{\text {air }} \\
[\AA]]\end{array}$} & \multirow{2}{*}{$\begin{array}{c}\chi_{\mathrm{I}} \\
{[\mathrm{eV}]}\end{array}$} & \multirow[t]{2}{*}{$\log g f$} & \multicolumn{4}{|c|}{ Line list ${ }^{a}$} & \multirow[t]{2}{*}{ Reference $^{b}$} \\
\hline & & & MRD & MPD & MRG & MPG & \\
\hline 5731.761 & 4.26 & -1.300 & $\bullet$ & $\bullet$ & & $\bullet$ & Sou08 \\
\hline 5732.296 & 4.99 & -1.560 & $\bullet$ & & & & Sou08 \\
\hline 5741.847 & 4.26 & -1.854 & $\bullet$ & & & & Sou08 \\
\hline 5759.262 & 4.65 & -2.070 & & & - & & Sou08 \\
\hline 5778.453 & 2.59 & -3.430 & & & $\bullet$ & & Sou08 \\
\hline 5784.658 & 3.40 & -2.532 & & & $\bullet$ & & Sou08 \\
\hline 5844.918 & 4.15 & -2.940 & & & - & & Sou08 \\
\hline 5849.683 & 3.69 & -2.990 & & & & - & Sou08 \\
\hline 5852.218 & 4.55 & -1.330 & $\bullet$ & & - & & Sou08 \\
\hline 5853.148 & 1.48 & -5.280 & & & $\bullet$ & $\bullet$ & Sou08 \\
\hline 5855.075 & 4.61 & -1.478 & $\bullet$ & & $\bullet$ & & Sou08 \\
\hline 5856.088 & 4.29 & -1.328 & & & $\bullet$ & & Sou08 \\
\hline 5858.778 & 4.22 & -2.260 & & & $\bullet$ & & Sou08 \\
\hline 5861.108 & 4.28 & -2.450 & & & $\bullet$ & $\bullet$ & Sou08 \\
\hline 5883.816 & 3.96 & -1.360 & - & - & & $\bullet$ & Sou08 \\
\hline 5902.472 & 4.59 & -1.810 & & & & • & Sou08 \\
\hline 5905.671 & 4.65 & -0.730 & $\bullet$ & $\bullet$ & $\bullet$ & $\bullet$ & Sou08 \\
\hline 5909.972 & 3.21 & -2.587 & $\bullet$ & & $\bullet$ & $\bullet$ & Sou08 \\
\hline 5916.247 & 2.45 & -2.994 & - & & $\bullet$ & $\bullet$ & Sou08 \\
\hline 5927.788 & 4.65 & -1.090 & - & & - & & Sou08 \\
\hline 5929.676 & 4.55 & -1.462 & $\bullet$ & & $\bullet$ & & Sou08 \\
\hline 5930.180 & 4.65 & -0.230 & $\bullet$ & - & $\bullet$ & & Sou08 \\
\hline 5934.654 & 3.93 & -1.170 & $\bullet$ & $\bullet$ & & $\bullet$ & Sou08 \\
\hline 5940.991 & 4.18 & -2.150 & & & & $\bullet$ & Sou08 \\
\hline 5952.718 & 3.98 & -1.440 & & & & $\bullet$ & Sou08 \\
\hline 5956.693 & 0.86 & -4.605 & - & - & - & - & Sou08 \\
\hline 6003.011 & 3.88 & -1.120 & $\bullet$ & $\bullet$ & - & $\bullet$ & Sou08 \\
\hline 6012.209 & 2.22 & -4.038 & & & & • & Sou08 \\
\hline 6019.365 & 3.57 & -3.360 & & & & - & Sou08 \\
\hline 6024.057 & 4.55 & -0.120 & $\bullet$ & $\bullet$ & & $\bullet$ & Sou08 \\
\hline 6027.051 & 4.08 & -1.089 & $\bullet$ & $\bullet$ & $\bullet$ & $\bullet$ & Sou08 \\
\hline 6056.004 & 4.73 & -0.460 & & - & & & Sou08 \\
\hline 6065.481 & 2.61 & -1.530 & & $\bullet$ & $\bullet$ & & Sou08 \\
\hline 6079.007 & 4.65 & -0.729 & $\bullet$ & $\bullet$ & $\bullet$ & $\bullet$ & Sou08 \\
\hline 6082.710 & 2.22 & -3.573 & - & & - & - & Sou08 \\
\hline 6093.642 & 4.61 & -1.500 & $\bullet$ & & $\bullet$ & $\bullet$ & Sou08 \\
\hline 6094.372 & 4.65 & -1.940 & & & & $\bullet$ & Sou08 \\
\hline 6096.664 & 3.98 & -1.930 & $\bullet$ & & & $\bullet$ & Sou08 \\
\hline 6098.243 & 4.56 & -1.880 & - & & - & $\bullet$ & Sou08 \\
\hline 6120.246 & 0.91 & -5.950 & & & - & $\bullet$ & Sou08 \\
\hline 6127.906 & 4.14 & -1.399 & - & $\bullet$ & & $\bullet$ & Sou08 \\
\hline 6136.614 & 2.45 & -1.400 & & $\bullet$ & & & Sou08 \\
\hline 6136.993 & 2.20 & -2.950 & & $\bullet$ & & & Sou08 \\
\hline 6137.691 & 2.59 & -1.403 & & $\bullet$ & & & Sou08 \\
\hline 6151.617 & 2.18 & -3.299 & $\bullet$ & $\bullet$ & & $\bullet$ & Sou08 \\
\hline 6165.359 & 4.14 & -1.474 & - & - & & - & Sou08 \\
\hline 6170.506 & 4.80 & -0.440 & $\bullet$ & $\bullet$ & & & Sou08 \\
\hline 6173.334 & 2.22 & -2.880 & - & - & - & - & Sou08 \\
\hline 6180.202 & 2.73 & -2.586 & - & & & & Sou08 \\
\hline 6187.989 & 3.94 & -1.720 & $\bullet$ & & $\bullet$ & $\bullet$ & Sou08 \\
\hline 6191.557 & 2.43 & -1.417 & & $\bullet$ & & & Sou08 \\
\hline 6199.506 & 2.56 & -4.430 & & & & $\bullet$ & Sou08 \\
\hline 6200.312 & 2.61 & -2.437 & $\bullet$ & - & & • & Sou08 \\
\hline 6213.429 & 2.22 & -2.482 & - & - & & $\bullet$ & Sou08 \\
\hline 6219.280 & 2.20 & -2.433 & $\bullet$ & • & & $\bullet$ & Sou08 \\
\hline 6220.779 & 3.88 & -2.460 & & & & $\bullet$ & Sou08 \\
\hline 6226.734 & 3.88 & -2.220 & $\bullet$ & & & & Sou08 \\
\hline 6229.225 & 2.85 & -2.805 & $\bullet$ & & $\bullet$ & $\bullet$ & Sou08 \\
\hline 6230.722 & 2.56 & -1.281 & - & $\bullet$ & & & Sou08 \\
\hline 6240.646 & 2.22 & -3.233 & - & - & - & $\bullet$ & Sou08 \\
\hline 6246.318 & 3.60 & -0.733 & $\bullet$ & $\bullet$ & $\bullet$ & & Sou08 \\
\hline 6252.555 & 2.40 & -1.687 & - & - & $\bullet$ & $\bullet$ & Sou08 \\
\hline 6265.132 & 2.18 & -2.550 & - & - & - & - & Sou08 \\
\hline
\end{tabular}


Table A4 - continued

\begin{tabular}{|c|c|c|c|c|c|c|c|}
\hline \multirow{2}{*}{$\begin{array}{l}\lambda_{\text {air }} \\
{[\AA \AA]}\end{array}$} & \multirow{2}{*}{$\begin{array}{c}\chi_{\mathrm{I}} \\
{[\mathrm{eV}]}\end{array}$} & \multirow[t]{2}{*}{$\log g f$} & \multicolumn{4}{|c|}{ Line list ${ }^{a}$} & \multirow[t]{2}{*}{ Reference $^{b}$} \\
\hline & & & MRD & MPD & MRG & MPG & \\
\hline 6270.223 & 2.86 & -2.464 & $\bullet$ & & $\bullet$ & $\bullet$ & Sou08 \\
\hline 6271.278 & 3.33 & -2.703 & & & $\bullet$ & $\bullet$ & Sou08 \\
\hline 6280.617 & 0.86 & -4.387 & & - & & & Sou08 \\
\hline 6290.543 & 2.59 & -4.330 & & & & $\bullet$ & Sou08 \\
\hline 6297.792 & 2.22 & -2.740 & & $\bullet$ & & $\bullet$ & Sou08 \\
\hline 6301.499 & 3.65 & -0.718 & & & • & $\bullet$ & Sou08 \\
\hline 6311.499 & 2.83 & -3.141 & $\bullet$ & & & $\bullet$ & Sou08 \\
\hline 6315.811 & 4.08 & -1.710 & - & & & $\bullet$ & Sou08 \\
\hline 6322.685 & 2.59 & -2.426 & $\bullet$ & - & - & $\bullet$ & Sou08 \\
\hline 6335.329 & 2.20 & -2.177 & $\bullet$ & $\bullet$ & $\bullet$ & $\bullet$ & Sou08 \\
\hline 6336.823 & 3.69 & -0.856 & - & $\bullet$ & - & $\bullet$ & Sou08 \\
\hline 6338.875 & 4.80 & -1.060 & $\bullet$ & & & & Sou08 \\
\hline 6344.147 & 2.43 & -2.923 & & $\bullet$ & & & Sou08 \\
\hline 6355.028 & 2.85 & -2.350 & & $\bullet$ & & & Sou08 \\
\hline 6380.743 & 4.19 & -1.376 & - & & & $\bullet$ & Sou08 \\
\hline 6393.600 & 2.43 & -1.432 & & - & - & & Sou08 \\
\hline 6400.316 & 0.91 & -4.318 & - & $\bullet$ & & & Sou08 \\
\hline 6411.648 & 3.65 & -0.595 & & $\bullet$ & & & Sou08 \\
\hline 6421.350 & 2.28 & -2.027 & - & $\bullet$ & & & Sou08 \\
\hline 6430.845 & 2.18 & -2.006 & - & $\bullet$ & & & Sou08 \\
\hline 6469.192 & 4.83 & -0.770 & - & • & & & Sou08 \\
\hline 6475.623 & 2.56 & -2.942 & • & $\bullet$ & & & Sou08 \\
\hline 6481.869 & 2.28 & -2.984 & • & $\bullet$ & & $\bullet$ & Sou08 \\
\hline 6494.980 & 2.40 & -1.273 & & - & & & Sou08 \\
\hline 6495.741 & 4.83 & -0.940 & $\bullet$ & & & & Sou08 \\
\hline 6496.465 & 4.80 & -0.570 & - & • & & - & Sou08 \\
\hline 6498.938 & 0.96 & -4.699 & - & & & - & Sou08 \\
\hline 6518.365 & 2.83 & -2.460 & & & & $\bullet$ & Sou08 \\
\hline 6533.928 & 4.56 & -1.460 & - & $\bullet$ & - & $\bullet$ & Sou08 \\
\hline 6546.237 & 2.76 & -1.536 & & - & - & - & Sou08 \\
\hline 6574.226 & 0.99 & -5.023 & $\bullet$ & & & & Sou08 \\
\hline 6581.209 & 1.48 & -4.679 & & & & $\bullet$ & Sou08 \\
\hline 6591.312 & 4.59 & -2.070 & & & $\bullet$ & $\bullet$ & Sou08 \\
\hline 6592.912 & 2.73 & -1.473 & $\bullet$ & $\bullet$ & & - & Sou08 \\
\hline 6593.869 & 2.43 & -2.422 & $\bullet$ & $\bullet$ & & $\bullet$ & Sou08 \\
\hline 6597.561 & 4.80 & -1.070 & & & & $\bullet$ & Sou08 \\
\hline 6608.025 & 2.28 & -4.030 & $\bullet$ & & & - & Sou08 \\
\hline 6609.109 & 2.56 & -2.692 & • & • & & $\bullet$ & Sou08 \\
\hline 6627.543 & 4.55 & -1.680 & $\bullet$ & & & & Sou08 \\
\hline 6633.412 & 4.83 & -1.490 & - & & & & Sou08 \\
\hline 6633.748 & 4.56 & -0.799 & • & & & & Sou08 \\
\hline 6648.079 & 1.01 & -5.429 & & & & $\bullet$ & Sou08 \\
\hline 6703.565 & 2.76 & -3.160 & • & & • & & Sou08 \\
\hline 6710.318 & 1.48 & -4.880 & • & & & & Sou08 \\
\hline 6713.742 & 4.80 & -1.600 & $\bullet$ & & - & & Sou08 \\
\hline 6716.236 & 4.58 & -1.920 & - & & & & Sou08 \\
\hline 6725.355 & 4.10 & -2.300 & & & $\bullet$ & - & Sou08 \\
\hline 6750.151 & 2.42 & -2.621 & • & • & - & • & Sou08 \\
\hline 6752.707 & 4.64 & -1.204 & • & $\bullet$ & & & Sou08 \\
\hline 6783.703 & 2.59 & -3.980 & - & • & $\bullet$ & & This work \\
\hline 6786.858 & 4.19 & -2.070 & • & • & - & • & This work \\
\hline 6793.258 & 4.08 & -2.326 & • & $\bullet$ & $\bullet$ & & This work \\
\hline 6793.619 & 4.80 & -1.329 & - & $\bullet$ & $\bullet$ & & This work \\
\hline 6796.123 & 4.14 & -2.530 & - & & - & & This work \\
\hline 6801.865 & 1.61 & -4.829 & & & & • & This work \\
\hline 6803.999 & 4.65 & -1.496 & - & $\bullet$ & & & This work \\
\hline 6804.270 & 4.58 & -1.813 & - & • & & & This work \\
\hline 6806.842 & 2.73 & -3.210 & $\bullet$ & - & $\bullet$ & - & This work \\
\hline 6810.262 & 4.61 & -0.986 & - & $\bullet$ & $\bullet$ & • & This work \\
\hline 6819.588 & 4.10 & -2.764 & - & & $\bullet$ & & This work \\
\hline 6820.371 & 4.64 & -1.320 & - & • & • & - & This work \\
\hline 6828.591 & 4.64 & -0.920 & • & $\bullet$ & $\bullet$ & • & This work \\
\hline 6833.225 & 4.64 & -2.080 & • & & $\bullet$ & & This work \\
\hline
\end{tabular}


Table A4 - continued

\begin{tabular}{|c|c|c|c|c|c|c|c|}
\hline \multirow{2}{*}{$\begin{array}{l}\lambda_{\text {air }} \\
{[\AA \AA]}\end{array}$} & \multirow{2}{*}{$\begin{array}{c}\chi_{\mathrm{I}} \\
{[\mathrm{eV}]}\end{array}$} & \multirow[t]{2}{*}{$\log g f$} & \multicolumn{4}{|c|}{ Line list ${ }^{a}$} & \multirow[t]{2}{*}{ Reference $^{b}$} \\
\hline & & & MRD & MPD & MRG & MPG & \\
\hline 6837.005 & 4.59 & -1.687 & - & - & $\bullet$ & - & This work \\
\hline 6838.827 & 5.84 & -0.361 & $\bullet$ & $\bullet$ & $\bullet$ & & This work \\
\hline 6839.829 & 2.56 & -3.450 & $\bullet$ & $\bullet$ & • & • & This work \\
\hline 6841.338 & 4.61 & -0.750 & $\bullet$ & • & • & & This work \\
\hline 6842.685 & 4.64 & -1.320 & - & - & - & - & This work \\
\hline 6843.655 & 4.55 & -0.930 & - & • & - & - & This work \\
\hline 6850.435 & 5.46 & -1.053 & $\bullet$ & & $\bullet$ & & This work \\
\hline 6851.635 & 1.61 & -5.320 & & & & $\bullet$ & This work \\
\hline 6854.823 & 4.59 & -1.926 & $\bullet$ & $\bullet$ & & & This work \\
\hline 6855.161 & 4.56 & -0.742 & $\bullet$ & $\bullet$ & $\bullet$ & & This work \\
\hline 6855.712 & 4.61 & -1.820 & - & - & - & & This work \\
\hline 6857.249 & 4.08 & -2.150 & $\bullet$ & - & - & $\bullet$ & This work \\
\hline 6858.148 & 4.61 & -0.930 & $\bullet$ & $\bullet$ & $\bullet$ & & This work \\
\hline 6859.479 & 2.85 & -4.520 & & & & - & This work \\
\hline 6861.937 & 2.42 & -3.890 & & $\bullet$ & & & This work \\
\hline 6862.480 & 4.56 & -1.570 & & $\bullet$ & & $\bullet$ & This work \\
\hline 6864.311 & 4.56 & -2.320 & & & & $\bullet$ & This work \\
\hline 6885.754 & 4.65 & -1.380 & & - & & - & This work \\
\hline 6911.511 & 2.42 & -4.040 & & & & - & This work \\
\hline 6916.680 & 4.15 & -1.450 & & & & $\bullet$ & This work \\
\hline 6933.617 & 2.43 & -3.580 & & $\bullet$ & & & This work \\
\hline 6945.204 & 2.42 & -2.482 & & & & $\bullet$ & This work \\
\hline 6947.488 & 4.58 & -1.611 & & • & & & This work \\
\hline 6951.245 & 4.56 & -0.908 & & - & & & This work \\
\hline 6971.932 & 3.02 & -3.340 & - & - & - & $\bullet$ & This work \\
\hline 6975.426 & 5.83 & -0.215 & - & • & & & This work \\
\hline 6977.428 & 4.59 & -1.564 & & • & & $\bullet$ & This work \\
\hline 6978.850 & 2.48 & -2.500 & $\bullet$ & - & - & & This work \\
\hline 6988.523 & 2.40 & -3.660 & & $\bullet$ & & - & This work \\
\hline 6999.883 & 4.10 & -1.560 & - & • & - & & This work \\
\hline 7000.614 & 4.14 & -2.386 & • & & - & • & This work \\
\hline 7007.965 & 4.18 & -2.060 & - & • & - & & This work \\
\hline 7011.343 & 4.59 & -1.316 & & • & $\bullet$ & $\bullet$ & This work \\
\hline 7014.986 & 2.45 & -4.250 & & & & $\bullet$ & This work \\
\hline 7016.055 & 2.42 & -3.210 & & $\bullet$ & & & This work \\
\hline 7022.390 & 4.30 & -2.290 & - & & - & • & This work \\
\hline 7022.952 & 4.19 & -1.250 & & • & & $\bullet$ & This work \\
\hline 7024.050 & 4.08 & -2.208 & - & $\bullet$ & $\bullet$ & & This work \\
\hline 7024.641 & 4.56 & -1.080 & & • & & & This work \\
\hline 7038.220 & 4.22 & -1.300 & - & $\bullet$ & - & & This work \\
\hline 7038.769 & 4.26 & -1.990 & $\bullet$ & • & - & & This work \\
\hline 7057.953 & 3.65 & -3.380 & & & & $\bullet$ & This work \\
\hline 7069.531 & 2.56 & -4.340 & & & & • & This work \\
\hline 7071.860 & 4.61 & -1.700 & & & & $\bullet$ & This work \\
\hline 7072.791 & 5.90 & -0.882 & - & & & & This work \\
\hline 7072.818 & 4.08 & -2.840 & & & - & & This work \\
\hline 7083.394 & 4.91 & -1.202 & - & • & - & $\bullet$ & This work \\
\hline 7086.724 & 3.60 & -2.356 & $\bullet$ & - & - & $\bullet$ & This work \\
\hline 7090.383 & 4.23 & -1.210 & $\bullet$ & $\bullet$ & $\bullet$ & & This work \\
\hline 7091.921 & 4.96 & -1.298 & • & & $\bullet$ & $\bullet$ & This work \\
\hline 7095.407 & 4.21 & -2.020 & $\bullet$ & - & - & & This work \\
\hline 7100.193 & 2.73 & -3.897 & & & & $\bullet$ & This work \\
\hline 7107.459 & 4.19 & -1.343 & & • & & & This work \\
\hline 7112.167 & 2.99 & -2.998 & $\bullet$ & - & - & $\bullet$ & This work \\
\hline 7114.548 & 2.69 & -4.010 & $\bullet$ & - & - & $\bullet$ & This work \\
\hline 7118.096 & 5.01 & -1.570 & $\bullet$ & & $\bullet$ & • & This work \\
\hline 7120.021 & 4.56 & -1.936 & $\bullet$ & & & & This work \\
\hline 7127.567 & 4.99 & -1.046 & $\bullet$ & $\bullet$ & $\bullet$ & & This work \\
\hline 7130.921 & 4.22 & -0.790 & $\bullet$ & - & - & & This work \\
\hline 7132.986 & 4.08 & -1.628 & - & & $\bullet$ & - & This work \\
\hline 7142.517 & 4.96 & -0.848 & & • & & • & This work \\
\hline 7145.306 & 4.61 & -1.145 & - & $\bullet$ & & & This work \\
\hline 7151.469 & 2.48 & -3.730 & • & • & • & $\bullet$ & This work \\
\hline
\end{tabular}


Table A4 - continued

\begin{tabular}{|c|c|c|c|c|c|c|c|}
\hline \multirow{2}{*}{$\begin{array}{l}\lambda_{\text {air }} \\
[\AA]]\end{array}$} & \multirow{2}{*}{$\begin{array}{c}\chi_{\mathrm{I}} \\
{[\mathrm{eV}]}\end{array}$} & \multirow[t]{2}{*}{$\log g f$} & \multicolumn{4}{|c|}{ Line list ${ }^{a}$} & \multirow[t]{2}{*}{ Reference $\mathrm{e}^{b}$} \\
\hline & & & MRD & MPD & MRG & MPG & \\
\hline 7155.630 & 5.01 & -0.725 & & - & & & This work \\
\hline 7162.343 & 5.02 & -1.064 & & & & - & This work \\
\hline 7179.994 & 1.48 & -4.780 & - & & - & & This work \\
\hline 7212.435 & 4.96 & -0.825 & - & & - & & This work \\
\hline 7219.682 & 4.08 & -1.690 & - & • & • & $\bullet$ & This work \\
\hline 7221.202 & 4.56 & -1.184 & - & • & - & & This work \\
\hline 7223.657 & 3.02 & -2.225 & & & & - & This work \\
\hline 7228.695 & 2.76 & -3.380 & - & - & $\bullet$ & & This work \\
\hline 7239.866 & 4.21 & -1.852 & & & & - & This work \\
\hline 7256.134 & 4.96 & -1.590 & & & & $\bullet$ & This work \\
\hline 7284.834 & 4.14 & -1.750 & - & & - & $\bullet$ & This work \\
\hline 7285.275 & 4.61 & -1.700 & • & & • & & This work \\
\hline 7288.736 & 4.22 & -1.035 & & $\bullet$ & & & This work \\
\hline 7306.562 & 4.18 & -1.740 & - & & - & • & This work \\
\hline 7311.074 & 4.28 & -0.967 & & & $\bullet$ & & This work \\
\hline 7376.480 & 6.00 & +0.089 & - & - & $\bullet$ & & This work \\
\hline 7381.333 & 5.35 & -1.087 & - & & - & & This work \\
\hline 7386.333 & 4.91 & -0.267 & • & & • & & This work \\
\hline 7396.507 & 4.99 & -1.640 & & & - & & This work \\
\hline 7401.683 & 4.19 & -1.599 & & & & • & This work \\
\hline 7411.153 & 4.28 & -0.299 & - & - & $\bullet$ & - & This work \\
\hline 7418.666 & 4.14 & -1.376 & • & $\bullet$ & $\bullet$ & $\bullet$ & This work \\
\hline 7421.559 & 4.64 & -1.800 & - & • & - & & This work \\
\hline 7430.538 & 2.59 & -3.860 & & $\bullet$ & & & This work \\
\hline 7430.855 & 4.61 & -1.539 & & $\bullet$ & & & This work \\
\hline 7435.591 & 5.31 & -0.716 & - & • & - & - & This work \\
\hline 7440.911 & 4.91 & -0.573 & & $\bullet$ & $\bullet$ & & This work \\
\hline 7443.022 & 4.19 & -1.820 & & $\bullet$ & & $\bullet$ & This work \\
\hline 7445.748 & 4.26 & -0.102 & & $\bullet$ & & & This work \\
\hline 7447.393 & 4.96 & -0.846 & $\bullet$ & - & $\bullet$ & $\bullet$ & This work \\
\hline 7453.997 & 4.19 & -2.410 & - & & & - & This work \\
\hline 7461.519 & 2.56 & -3.580 & - & $\bullet$ & - & - & This work \\
\hline 7463.382 & 5.06 & -1.720 & - & & • & & This work \\
\hline 7464.293 & 5.41 & -1.066 & - & & $\bullet$ & & This work \\
\hline 7472.750 & 5.35 & -0.994 & - & & & & This work \\
\hline 7473.554 & 4.61 & -1.870 & - & & - & & This work \\
\hline 7477.506 & 3.88 & -3.045 & & & & $\bullet$ & This work \\
\hline 7484.297 & 5.09 & -1.700 & - & & & & This work \\
\hline 7491.647 & 4.30 & -0.900 & - & - & - & & This work \\
\hline 7495.065 & 4.22 & +0.052 & & $\bullet$ & & & This work \\
\hline 7498.530 & 4.14 & -2.250 & - & • & - & $\bullet$ & This work \\
\hline 7504.270 & 5.39 & -1.006 & - & & - & & This work \\
\hline 7506.013 & 5.06 & -1.219 & - & & & & This work \\
\hline 7507.265 & 4.42 & -1.485 & - & - & - & - & This work \\
\hline 7511.018 & 4.18 & +0.099 & - & $\bullet$ & - & • & This work \\
\hline 7514.198 & 5.39 & -0.874 & - & & $\bullet$ & $\bullet$ & This work \\
\hline 7531.143 & 4.37 & -0.951 & & & & - & This work \\
\hline 7540.429 & 2.73 & -3.850 & - & & - & • & This work \\
\hline 7547.896 & 5.10 & -1.350 & • & & $\bullet$ & $\bullet$ & This work \\
\hline 7551.104 & 5.09 & -1.630 & - & & $\bullet$ & & This work \\
\hline 7559.719 & 5.06 & -1.080 & - & • & - & & This work \\
\hline 7563.010 & 4.83 & -2.047 & & & & - & This work \\
\hline 7568.898 & 4.28 & -0.773 & - & $\bullet$ & - & - & This work \\
\hline 7573.413 & 6.58 & +0.302 & - & & - & & This work \\
\hline 7582.121 & 4.96 & -1.750 & • & & • & & This work \\
\hline 7583.788 & 3.02 & -1.885 & - & $\bullet$ & $\bullet$ & $\bullet$ & This work \\
\hline 7586.017 & 4.31 & -0.470 & - & & $\bullet$ & $\bullet$ & This work \\
\hline 7588.844 & 5.10 & -1.672 & - & & & & This work \\
\hline 7620.513 & 4.73 & -0.664 & & $\bullet$ & & & This work \\
\hline 7689.036 & 5.10 & -1.370 & & & & • & This work \\
\hline 7710.363 & 4.22 & -1.113 & & - & & - & This work \\
\hline 7719.048 & 5.03 & -1.153 & - & $\bullet$ & - & $\bullet$ & This work \\
\hline 7723.207 & 2.28 & -3.617 & & • & & • & This work \\
\hline
\end{tabular}


Table A4 - continued

\begin{tabular}{|c|c|c|c|c|c|c|c|}
\hline \multirow{2}{*}{$\begin{array}{l}\lambda_{\text {air }} \\
[\AA]]\end{array}$} & \multirow{2}{*}{$\begin{array}{c}\chi_{\mathrm{I}} \\
{[\mathrm{eV}]}\end{array}$} & \multirow[t]{2}{*}{$\log g f$} & \multicolumn{4}{|c|}{ Line list ${ }^{a}$} & \multirow[t]{2}{*}{ Reference $^{b}$} \\
\hline & & & MRD & MPD & MRG & MPG & \\
\hline 7733.723 & 5.06 & -1.536 & - & & - & - & This work \\
\hline 7745.513 & 5.09 & -1.170 & $\bullet$ & - & - & $\bullet$ & This work \\
\hline 7746.595 & 5.06 & -1.284 & $\bullet$ & $\bullet$ & $\bullet$ & $\bullet$ & This work \\
\hline 7748.269 & 2.95 & -1.751 & & - & - & - & This work \\
\hline 7751.108 & 4.99 & -0.754 & $\bullet$ & $\bullet$ & $\bullet$ & $\bullet$ & This work \\
\hline 7780.556 & 4.47 & +0.030 & - & - & - & - & This work \\
\hline 7802.473 & 5.09 & -1.335 & $\bullet$ & & - & $\bullet$ & This work \\
\hline 7807.908 & 4.99 & -0.542 & $\bullet$ & $\bullet$ & $\bullet$ & $\bullet$ & This work \\
\hline 7832.195 & 4.43 & +0.112 & $\bullet$ & $\bullet$ & - & $\bullet$ & This work \\
\hline 7844.558 & 4.83 & -1.810 & $\bullet$ & & $\bullet$ & $\bullet$ & This work \\
\hline 7855.399 & 5.06 & -1.017 & & $\bullet$ & & & This work \\
\hline 7869.609 & 4.37 & -1.880 & & $\bullet$ & & & This work \\
\hline 7879.756 & 5.03 & -1.650 & - & & - & - & This work \\
\hline 7912.866 & 0.86 & -4.848 & & $\bullet$ & & & This work \\
\hline 7937.139 & 4.31 & +0.228 & & • & & - & This work \\
\hline 7941.087 & 3.27 & -2.286 & - & - & - & $\bullet$ & This work \\
\hline 7945.846 & 4.39 & +0.227 & & $\bullet$ & & - & This work \\
\hline 7954.934 & 2.99 & -3.675 & $\bullet$ & & & & This work \\
\hline 7959.142 & 5.03 & -1.212 & - & - & - & & This work \\
\hline 7998.944 & 4.37 & +0.151 & & - & & $\bullet$ & This work \\
\hline 8028.312 & 4.47 & -0.689 & & $\bullet$ & & & This work \\
\hline 8046.046 & 4.42 & +0.032 & & • & & & This work \\
\hline 8047.617 & 0.86 & -4.787 & $\bullet$ & - & $\bullet$ & $\bullet$ & This work \\
\hline 8075.149 & 0.91 & -5.062 & & - & - & - & This work \\
\hline 8085.171 & 4.45 & -0.121 & & $\bullet$ & & $\bullet$ & This work \\
\hline 8089.353 & 5.07 & -1.147 & - & & & & This work \\
\hline 8090.325 & 4.58 & -1.912 & $\bullet$ & & & & This work \\
\hline 8096.875 & 4.08 & -1.776 & & $\bullet$ & & $\bullet$ & This work \\
\hline 8198.920 & 4.43 & -0.566 & & - & & & This work \\
\hline 8204.936 & 0.96 & -5.058 & & $\bullet$ & & & This work \\
\hline 8207.741 & 4.45 & -0.856 & $\bullet$ & $\bullet$ & $\bullet$ & $\bullet$ & This work \\
\hline 8220.377 & 4.32 & +0.275 & & & & $\bullet$ & This work \\
\hline 8239.127 & 2.42 & -3.180 & & & & $\bullet$ & This work \\
\hline 8248.128 & 4.37 & -0.892 & & $\bullet$ & & $\bullet$ & This work \\
\hline 8293.512 & 3.30 & -2.175 & & $\bullet$ & & $\bullet$ & This work \\
\hline 8327.055 & 2.20 & -1.525 & & • & & - & This work \\
\hline 8340.502 & 5.07 & -1.701 & - & & - & & This work \\
\hline 8342.856 & 4.99 & -1.468 & $\bullet$ & & $\bullet$ & & This work \\
\hline 8349.045 & 0.91 & -5.705 & & & & $\bullet$ & This work \\
\hline 8358.520 & 2.99 & -3.145 & - & & - & & This work \\
\hline 8360.793 & 4.47 & -1.688 & & & & $\bullet$ & This work \\
\hline 8365.631 & 3.25 & -2.047 & - & & - & - & This work \\
\hline 8387.771 & 2.18 & -1.493 & & $\bullet$ & & $\bullet$ & This work \\
\hline 8404.395 & 5.79 & -0.705 & $\bullet$ & & & & This work \\
\hline 8419.271 & 6.18 & -0.231 & $\bullet$ & & & & This work \\
\hline 8422.913 & 4.14 & -2.002 & & - & & & This work \\
\hline 8424.141 & 4.96 & -1.156 & - & $\bullet$ & $\bullet$ & & This work \\
\hline 8439.570 & 4.55 & -0.591 & & $\bullet$ & & $\bullet$ & This work \\
\hline 8447.636 & 0.96 & -6.699 & & & & - & This work \\
\hline 8453.657 & 5.54 & -1.043 & - & & - & & This work \\
\hline 8468.406 & 2.22 & -2.072 & & $\bullet$ & & & This work \\
\hline 8471.743 & 4.96 & -1.019 & & • & & $\bullet$ & This work \\
\hline 8481.980 & 4.19 & -1.999 & & • & & • & This work \\
\hline 8514.071 & 2.20 & -2.229 & $\bullet$ & $\bullet$ & $\bullet$ & $\bullet$ & This work \\
\hline 8515.108 & 3.02 & -2.073 & - & • & $\bullet$ & & This work \\
\hline 8517.305 & 6.13 & -0.259 & - & & & & This work \\
\hline 8526.669 & 4.91 & -0.760 & & - & & - & This work \\
\hline 8571.804 & 5.01 & -1.391 & & & & $\bullet$ & This work \\
\hline 8582.257 & 2.99 & -2.134 & $\bullet$ & $\bullet$ & $\bullet$ & - & This work \\
\hline 8592.951 & 4.96 & -1.086 & & - & & $\bullet$ & This work \\
\hline 8598.828 & 4.39 & -1.089 & & $\bullet$ & & $\bullet$ & This work \\
\hline 8607.080 & 5.01 & -1.557 & & & & $\bullet$ & This work \\
\hline 8611.803 & 2.85 & -1.926 & $\bullet$ & $\bullet$ & $\bullet$ & $\bullet$ & This work \\
\hline
\end{tabular}


Table A4 - continued

\begin{tabular}{|c|c|c|c|c|c|c|c|}
\hline \multirow{2}{*}{$\begin{array}{l}\lambda_{\text {air }} \\
{[\AA]}\end{array}$} & \multirow{2}{*}{$\begin{array}{c}\chi_{\mathrm{I}} \\
{[\mathrm{eV}]}\end{array}$} & \multirow[t]{2}{*}{$\log g f$} & \multicolumn{4}{|c|}{ Line list ${ }^{a}$} & \multirow[t]{2}{*}{ Reference $^{b}$} \\
\hline & & & MRD & MPD & MRG & MPG & \\
\hline 8613.939 & 4.99 & -1.246 & & & & - & This work \\
\hline 8616.280 & 4.91 & -0.707 & & - & & • & This work \\
\hline 8621.601 & 2.95 & -2.321 & - & - & - & - & This work \\
\hline 8632.413 & 4.10 & -2.409 & & & & $\bullet$ & This work \\
\hline 8667.366 & 2.45 & -4.939 & & & & • & This work \\
\hline 8674.746 & 2.83 & -1.800 & - & - & - & - & This work \\
\hline 8678.930 & 2.45 & -5.418 & & & & $\bullet$ & This work \\
\hline 8679.632 & 4.97 & -1.276 & - & - & - & & This work \\
\hline 8688.623 & 2.18 & -1.212 & $\bullet$ & $\bullet$ & • & - & This work \\
\hline 8698.706 & 2.99 & -3.442 & & & & • & This work \\
\hline 8699.454 & 4.96 & -0.380 & & • & & • & This work \\
\hline 8710.392 & 4.91 & -0.532 & & • & - & & This work \\
\hline 8713.187 & 2.95 & -2.467 & & • & & • & This work \\
\hline 8729.143 & 3.41 & -2.872 & & - & & $\bullet$ & This work \\
\hline 8747.425 & 3.02 & -3.174 & - & • & - & - & This work \\
\hline 8757.187 & 2.85 & -2.059 & - & - & $\bullet$ & $\bullet$ & This work \\
\hline 8763.966 & 4.65 & -0.146 & - & - & - & $\bullet$ & This work \\
\hline 8784.440 & 4.96 & -1.593 & - & • & • & - & This work \\
\hline 8793.341 & 4.61 & -0.092 & & - & & & This work \\
\hline 8796.484 & 4.96 & -1.229 & $\bullet$ & & - & $\bullet$ & This work \\
\hline 8804.624 & 2.28 & -3.234 & - & - & $\bullet$ & • & This work \\
\hline 8824.219 & 2.20 & -1.540 & - & • & • & • & This work \\
\hline 8828.091 & 4.96 & -2.240 & & & & • & This work \\
\hline 8834.016 & 4.22 & -2.590 & & & & - & This work \\
\hline 8838.428 & 2.86 & -2.050 & - & • & • & • & This work \\
\hline 8846.740 & 5.01 & -0.781 & - & - & $\bullet$ & - & This work \\
\hline 8863.587 & 4.97 & -1.519 & & & & - & This work \\
\hline 8866.931 & 4.55 & +0.083 & - & • & • & • & This work \\
\hline 8868.430 & 3.02 & -2.909 & $\bullet$ & - & $\bullet$ & - & This work \\
\hline 8876.024 & 5.02 & -1.052 & - & - & $\bullet$ & & This work \\
\hline 8878.250 & 2.99 & -3.383 & & & & $\bullet$ & This work \\
\hline 8897.899 & 4.91 & -1.937 & & & & - & This work \\
\hline 8902.924 & 4.99 & -2.106 & & & & $\bullet$ & This work \\
\hline 8920.013 & 5.06 & -0.415 & - & - & $\bullet$ & & This work \\
\hline 8922.650 & 4.99 & -1.698 & - & & • & • & This work \\
\hline 8929.075 & 5.09 & -0.893 & & - & & • & This work \\
\hline 8931.776 & 3.05 & -3.216 & & & & $\bullet$ & This work \\
\hline 8943.065 & 2.83 & -3.346 & • & - & & - & This work \\
\hline 8945.189 & 5.03 & -0.220 & - & & $\bullet$ & - & This work \\
\hline 8946.260 & 2.85 & -3.509 & & & & - & This work \\
\hline 8950.188 & 4.15 & -2.425 & & & & $\bullet$ & This work \\
\hline 8975.401 & 2.99 & -2.233 & - & & $\bullet$ & & This work \\
\hline 8978.198 & 3.41 & -3.457 & & & & $\bullet$ & This work \\
\hline 8984.886 & 5.10 & -0.922 & - & & - & $\bullet$ & This work \\
\hline 8994.628 & 3.27 & -3.189 & & & & • & This work \\
\hline 8999.556 & 2.83 & -1.321 & & - & & - & This work \\
\hline 9010.592 & 2.61 & -2.953 & & & & - & This work \\
\hline 9013.977 & 2.28 & -3.839 & & & & • & This work \\
\hline 9019.744 & 5.10 & -0.988 & & & & - & This work \\
\hline 9057.971 & 3.05 & -4.467 & & & & $\bullet$ & This work \\
\hline 9079.579 & 4.65 & -0.809 & & - & & & This work \\
\hline 9080.386 & 4.96 & -1.104 & & - & & & This work \\
\hline 9084.184 & 4.26 & -2.240 & & & & • & This work \\
\hline 9089.404 & 2.95 & -1.675 & & & & • & This work \\
\hline 9103.635 & 4.18 & -1.921 & & & & • & This work \\
\hline 9210.024 & 2.85 & -2.404 & - & & & & This work \\
\hline 9214.499 & 4.91 & -0.743 & & - & & & This work \\
\hline 9258.267 & 4.61 & -0.725 & - & • & & & This work \\
\hline 9259.005 & 4.91 & -0.749 & & $\bullet$ & & & This work \\
\hline 9800.308 & 5.09 & -0.453 & & $\bullet$ & & & This work \\
\hline 9811.504 & 5.01 & -1.362 & & & & - & This work \\
\hline 9820.241 & 2.42 & -5.073 & & & & - & This work \\
\hline 9834.185 & 4.99 & -1.214 & - & & & & This work \\
\hline
\end{tabular}


Table A4 - continued

\begin{tabular}{|c|c|c|c|c|c|c|c|}
\hline \multirow{2}{*}{$\begin{array}{l}\lambda_{\text {air }} \\
{[\AA ̊]}\end{array}$} & \multirow{2}{*}{$\begin{array}{c}\chi_{\mathrm{I}} \\
{[\mathrm{eV}]}\end{array}$} & \multirow[t]{2}{*}{$\log g f$} & \multicolumn{4}{|c|}{ Line list ${ }^{a}$} & \multirow[t]{2}{*}{ Reference $^{b}$} \\
\hline & & & MRD & MPD & MRG & MPG & \\
\hline 9847.457 & 4.58 & -2.305 & & & & - & This work \\
\hline 9861.734 & 5.06 & -0.142 & $\bullet$ & & & & This work \\
\hline 9868.186 & 5.09 & -0.979 & $\bullet$ & $\bullet$ & & $\bullet$ & This work \\
\hline 9881.522 & 4.58 & -1.711 & - & & & - & This work \\
\hline 9886.081 & 5.01 & -1.953 & & & & $\bullet$ & This work \\
\hline 9889.035 & 5.03 & -0.446 & - & - & - & - & This work \\
\hline 9913.180 & 4.99 & -1.266 & $\bullet$ & & - & $\bullet$ & This work \\
\hline 9924.388 & 3.55 & -3.127 & $\bullet$ & & & & This work \\
\hline 9944.207 & 5.01 & -1.338 & $\bullet$ & $\bullet$ & - & - & This work \\
\hline 9951.157 & 5.39 & -1.267 & $\bullet$ & $\bullet$ & $\bullet$ & $\bullet$ & This work \\
\hline 9953.470 & 5.45 & -1.309 & & & & - & This work \\
\hline 9970.233 & 3.02 & -4.818 & & & & - & This work \\
\hline 9977.641 & 5.06 & -1.660 & - & & - & & This work \\
\hline 9980.463 & 5.03 & -1.379 & - & & - & - & This work \\
\hline 9999.924 & 5.50 & -1.421 & & & & - & This work \\
\hline 10041.472 & 5.01 & -1.772 & & & & - & This work \\
\hline 10065.045 & 4.83 & -0.289 & $\bullet$ & $\bullet$ & - & - & This work \\
\hline 10081.393 & 2.42 & -4.537 & & & & $\bullet$ & And16 \\
\hline 10086.242 & 2.95 & -4.054 & & & & $\bullet$ & This work \\
\hline 10089.776 & 5.45 & -1.247 & & & & - & This work \\
\hline 10114.014 & 2.76 & -3.692 & $\bullet$ & $\bullet$ & $\bullet$ & $\bullet$ & This work \\
\hline 10137.100 & 5.09 & -1.708 & $\bullet$ & & - & $\bullet$ & And16 \\
\hline 10142.844 & 5.06 & -1.510 & $\bullet$ & & $\bullet$ & & And16 \\
\hline 10145.561 & 4.80 & -0.177 & - & - & & & This work \\
\hline 10155.162 & 2.18 & -4.226 & $\bullet$ & $\bullet$ & $\bullet$ & $\bullet$ & And16 \\
\hline 10167.468 & 2.20 & -4.117 & - & $\bullet$ & - & - & And16 \\
\hline 10195.105 & 2.73 & -3.580 & $\bullet$ & & $\bullet$ & $\bullet$ & And16 \\
\hline 10216.313 & 4.73 & -0.063 & & $\bullet$ & & $\bullet$ & This work \\
\hline 10218.408 & 3.07 & -2.760 & & - & & $\bullet$ & This work \\
\hline 10227.994 & 6.12 & -0.354 & $\bullet$ & & $\bullet$ & & And16 \\
\hline 10230.795 & 6.12 & -0.339 & $\bullet$ & & $\bullet$ & $\bullet$ & And16 \\
\hline 10252.551 & 5.83 & -1.026 & & & & - & This work \\
\hline 10262.471 & 5.48 & -1.613 & & & & $\bullet$ & This work \\
\hline 10265.217 & 2.22 & -4.537 & $\bullet$ & & $\bullet$ & $\bullet$ & And16 \\
\hline 10307.454 & 4.59 & -2.067 & & & & - & This work \\
\hline 10332.327 & 3.64 & -2.938 & $\bullet$ & & $\bullet$ & $\bullet$ & And16 \\
\hline 10333.184 & 4.59 & -2.585 & & & & $\bullet$ & This work \\
\hline 10340.885 & 2.20 & -3.577 & $\bullet$ & $\bullet$ & $\bullet$ & $\bullet$ & And16 \\
\hline 10347.965 & 5.39 & -0.551 & - & - & - & $\bullet$ & And16 \\
\hline 10353.804 & 5.39 & -0.819 & - & $\bullet$ & - & $\bullet$ & And16 \\
\hline 10364.062 & 5.45 & -0.960 & $\bullet$ & & $\bullet$ & $\bullet$ & And16 \\
\hline 10378.999 & 2.22 & -4.148 & & - & & & And16 \\
\hline 10388.744 & 5.45 & -1.468 & & & & $\bullet$ & And16 \\
\hline 10395.794 & 2.18 & -3.393 & $\bullet$ & & & $\bullet$ & This work \\
\hline 10423.027 & 2.69 & -3.616 & & $\bullet$ & & $\bullet$ & This work \\
\hline 10423.743 & 3.07 & -2.918 & - & - & - & - & And16 \\
\hline 10435.355 & 4.73 & -1.945 & & & & $\bullet$ & This work \\
\hline 10469.652 & 3.88 & -1.184 & - & - & & $\bullet$ & This work \\
\hline 10532.234 & 3.93 & -1.480 & - & - & $\bullet$ & - & And16 \\
\hline 10555.649 & 5.45 & -1.108 & - & & - & $\bullet$ & And16 \\
\hline 10577.139 & 3.30 & -3.136 & $\bullet$ & $\bullet$ & $\bullet$ & $\bullet$ & And16 \\
\hline 10611.686 & 6.17 & +0.021 & $\bullet$ & • & - & $\bullet$ & And16 \\
\hline 10616.721 & 3.27 & -3.127 & $\bullet$ & $\bullet$ & $\bullet$ & $\bullet$ & And16 \\
\hline 10674.070 & 6.17 & -0.466 & $\bullet$ & & $\bullet$ & $\bullet$ & And16 \\
\hline 10742.550 & 3.64 & -3.629 & & & & - & This work \\
\hline 10753.004 & 3.96 & -1.845 & $\bullet$ & $\bullet$ & & - & This work \\
\hline 10754.753 & 2.83 & -4.523 & & & & - & This work \\
\hline 10780.694 & 3.24 & -3.289 & $\bullet$ & & $\bullet$ & $\bullet$ & And16 \\
\hline 10783.050 & 3.11 & -2.567 & - & - & & - & This work \\
\hline 10818.274 & 3.96 & -1.948 & - & - & - & $\bullet$ & And16 \\
\hline 10849.465 & 5.54 & -1.444 & & & & $\bullet$ & This work \\
\hline 10863.518 & 4.73 & -0.895 & & & & $\bullet$ & This work \\
\hline 10881.758 & 2.85 & -3.604 & & - & & - & This work \\
\hline
\end{tabular}


Table A4 - continued

\begin{tabular}{|c|c|c|c|c|c|c|c|}
\hline \multirow{2}{*}{$\begin{array}{l}\lambda_{\text {air }} \\
[\AA]]\end{array}$} & \multirow{2}{*}{$\begin{array}{c}\chi_{\mathrm{I}} \\
{[\mathrm{eV}]}\end{array}$} & \multirow[t]{2}{*}{$\log g f$} & \multicolumn{4}{|c|}{ Line list ${ }^{a}$} & \multirow[t]{2}{*}{ Reference $^{b}$} \\
\hline & & & MRD & MPD & MRG & MPG & \\
\hline 10884.262 & 3.93 & -1.925 & $\bullet$ & - & & • & This work \\
\hline 10888.606 & 2.28 & -5.433 & & & & $\bullet$ & This work \\
\hline 10896.299 & 3.07 & -2.694 & - & & & • & This work \\
\hline 11026.788 & 3.94 & -2.805 & & & & - & And16 \\
\hline 11045.599 & 5.59 & -0.624 & & & & $\bullet$ & This work \\
\hline 11071.712 & 3.07 & -4.281 & & & & • & This work \\
\hline 12053.082 & 4.56 & -1.543 & $\bullet$ & & $\bullet$ & $\bullet$ & And16 \\
\hline 12283.298 & 6.17 & -0.537 & $\bullet$ & & $\bullet$ & $\bullet$ & And16 \\
\hline 12485.492 & 2.42 & -5.379 & & & & $\bullet$ & This work \\
\hline 12510.519 & 4.96 & -1.605 & • & & • & $\bullet$ & And16 \\
\hline 12545.946 & 4.08 & -3.485 & & & & - & This work \\
\hline 12556.996 & 2.28 & -3.626 & $\bullet$ & & - & • & And16 \\
\hline 12615.928 & 4.64 & -1.517 & $\bullet$ & & • & & And16 \\
\hline 12638.703 & 4.56 & -0.783 & & - & & & This work \\
\hline 12648.741 & 4.61 & -1.140 & & $\bullet$ & & & And16 \\
\hline 12789.450 & 5.01 & -1.514 & & & & - & This work \\
\hline 12807.152 & 3.64 & -2.452 & - & - & - & - & And16 \\
\hline 12808.243 & 4.99 & -1.362 & • & & • & • & And16 \\
\hline 12824.859 & 3.02 & -3.835 & - & & - & - & And16 \\
\hline 12879.766 & 2.28 & -3.458 & - & & & $\bullet$ & This work \\
\hline 12933.006 & 5.02 & -1.548 & - & & $\bullet$ & - & And16 \\
\hline 12934.666 & 5.39 & -0.948 & & & $\bullet$ & $\bullet$ & And16 \\
\hline 12946.532 & 3.25 & -4.754 & & & & $\bullet$ & This work \\
\hline 13014.841 & 5.45 & -1.693 & & & & $\bullet$ & And16 \\
\hline 13098.876 & 5.01 & -1.290 & & & & $\bullet$ & This work \\
\hline 13352.173 & 5.31 & -0.521 & - & & - & & And16 \\
\hline 14939.644 & 6.47 & -0.153 & & & & - & This work \\
\hline 14979.696 & 6.17 & -0.451 & & & & $\bullet$ & And16 \\
\hline 14982.801 & 6.26 & -0.495 & - & & - & & And16 \\
\hline 14988.778 & 6.17 & +0.186 & - & & & & This work \\
\hline 15013.771 & 6.22 & +0.087 & & & & $\bullet$ & This work \\
\hline 15017.700 & 6.22 & +0.062 & - & $\bullet$ & - & - & And16 \\
\hline 15160.503 & 6.34 & -0.253 & - & & $\bullet$ & & This work \\
\hline 15176.713 & 5.92 & -0.497 & - & & - & & And16 \\
\hline 15194.490 & 2.22 & -4.815 & $\bullet$ & & $\bullet$ & $\bullet$ & And16 \\
\hline 15201.562 & 6.31 & -0.161 & & & & $\bullet$ & And16 \\
\hline 15207.526 & 5.39 & +0.323 & $\bullet$ & $\bullet$ & $\bullet$ & - & And16 \\
\hline 15219.618 & 5.59 & -0.825 & - & & - & $\bullet$ & And16 \\
\hline 15224.729 & 5.96 & -0.315 & - & & - & - & And16 \\
\hline 15239.712 & 6.42 & -0.032 & & & $\bullet$ & - & And16 \\
\hline 15244.973 & 5.59 & -0.072 & & & & $\bullet$ & This work \\
\hline 15293.135 & 6.31 & +0.143 & - & & & & This work \\
\hline 15294.560 & 5.31 & +0.719 & - & $\bullet$ & & $\bullet$ & This work \\
\hline 15301.557 & 5.92 & -0.687 & • & & - & $\bullet$ & This work \\
\hline 15335.383 & 5.41 & +0.088 & - & & & & And16 \\
\hline 15343.788 & 5.65 & -0.582 & $\bullet$ & $\bullet$ & $\bullet$ & - & This work \\
\hline 15348.966 & 5.95 & -1.260 & - & & $\bullet$ & & This work \\
\hline 15375.346 & 5.92 & -0.991 & • & & • & • & And16 \\
\hline 15394.673 & 5.62 & +0.008 & - & & - & $\bullet$ & And16 \\
\hline 15395.718 & 5.62 & -0.126 & - & - & - & - & And16 \\
\hline 15514.279 & 6.29 & -0.473 & - & & & & And16 \\
\hline 15522.607 & 6.32 & -1.118 & - & & & - & And16 \\
\hline 15524.308 & 5.79 & -0.881 & - & & - & - & And16 \\
\hline 15531.751 & 5.64 & -0.243 & • & • & - & $\bullet$ & And16 \\
\hline 15534.245 & 5.64 & -0.382 & - & $\bullet$ & • & • & And 16 \\
\hline 15542.079 & 5.64 & -0.337 & - & $\bullet$ & - & $\bullet$ & And16 \\
\hline 15550.436 & 6.32 & -0.102 & - & & - & $\bullet$ & And16 \\
\hline 15551.433 & 6.35 & -0.371 & - & & - & - & And 16 \\
\hline 15560.784 & 6.35 & -0.475 & - & & - & & And16 \\
\hline 15565.222 & 6.32 & -0.557 & - & & - & & And16 \\
\hline 15566.725 & 6.35 & -0.681 & & & $\bullet$ & • & And16 \\
\hline 15588.259 & 6.37 & +0.419 & - & $\bullet$ & - & & And 16 \\
\hline 15590.046 & 6.24 & -0.829 & - & & & - & And16 \\
\hline
\end{tabular}


Table A4 - continued

\begin{tabular}{|c|c|c|c|c|c|c|c|}
\hline \multirow{2}{*}{$\begin{array}{l}\lambda_{\text {air }} \\
{[\AA ̊]}\end{array}$} & \multirow{2}{*}{$\begin{array}{c}\chi_{\mathrm{I}} \\
{[\mathrm{eV}]}\end{array}$} & \multirow[t]{2}{*}{$\log g f$} & \multicolumn{4}{|c|}{ Line list ${ }^{a}$} & \multirow[t]{2}{*}{ Reference $^{b}$} \\
\hline & & & MRD & MPD & MRG & MPG & \\
\hline 15591.490 & 6.24 & +0.874 & $\bullet$ & $\bullet$ & $\bullet$ & $\bullet$ & And16 \\
\hline 15593.749 & 5.03 & -1.922 & $\bullet$ & & $\bullet$ & & And16 \\
\hline 15598.869 & 6.24 & -0.236 & & & $\bullet$ & & And16 \\
\hline 15604.220 & 6.24 & +0.538 & $\bullet$ & $\bullet$ & $\bullet$ & $\bullet$ & This work \\
\hline 15611.145 & 3.41 & -3.768 & & & $\bullet$ & $\bullet$ & And16 \\
\hline 15621.654 & 5.54 & +0.589 & - & - & & $\bullet$ & This work \\
\hline 15645.016 & 6.31 & -0.390 & $\bullet$ & & $\bullet$ & $\bullet$ & This work \\
\hline 15648.510 & 5.43 & -0.599 & - & $\bullet$ & - & $\bullet$ & And16 \\
\hline 15652.871 & 6.25 & -0.161 & $\bullet$ & $\bullet$ & - & & And16 \\
\hline 15662.013 & 5.83 & +0.371 & $\bullet$ & $\bullet$ & $\bullet$ & $\bullet$ & And16 \\
\hline 15665.240 & 5.98 & -0.337 & - & - & & & This work \\
\hline 15682.513 & 6.37 & -0.265 & $\bullet$ & & $\bullet$ & $\bullet$ & And16 \\
\hline 15691.853 & 6.25 & +0.649 & $\bullet$ & & & & And16 \\
\hline 15723.586 & 5.62 & -0.143 & - & $\bullet$ & - & & And16 \\
\hline 15731.412 & 6.45 & -0.337 & & & $\bullet$ & & And16 \\
\hline 15733.509 & 6.25 & -0.978 & - & & & & And16 \\
\hline 15788.996 & 6.25 & +0.490 & $\bullet$ & & & & And16 \\
\hline 15920.642 & 6.26 & +0.366 & $\bullet$ & & $\bullet$ & & This work \\
\hline 15928.158 & 5.95 & -0.680 & - & & $\bullet$ & - & And16 \\
\hline 15929.472 & 6.31 & -0.383 & $\bullet$ & & & $\bullet$ & And16 \\
\hline 15934.017 & 6.31 & -0.294 & $\bullet$ & & $\bullet$ & $\bullet$ & And16 \\
\hline 15938.918 & 6.37 & +0.065 & & & & $\bullet$ & This work \\
\hline 15940.918 & 5.81 & -1.594 & $\bullet$ & & & $\bullet$ & And16 \\
\hline 15941.848 & 6.36 & +0.265 & $\bullet$ & $\bullet$ & $\bullet$ & & And16 \\
\hline 15962.558 & 6.42 & -0.078 & $\bullet$ & & $\bullet$ & $\bullet$ & This work \\
\hline 15964.865 & 5.92 & +0.279 & - & - & $\bullet$ & $\bullet$ & And16 \\
\hline 15980.725 & 6.26 & +0.958 & $\bullet$ & $\bullet$ & & & And16 \\
\hline 16009.610 & 5.43 & -0.470 & $\bullet$ & - & & & And16 \\
\hline 16040.654 & 5.87 & +0.317 & - & & - & & And16 \\
\hline 16051.734 & 6.26 & -0.942 & $\bullet$ & & $\bullet$ & & And16 \\
\hline 16070.180 & 5.96 & -0.569 & $\bullet$ & & $\bullet$ & & And16 \\
\hline 16071.397 & 6.27 & +0.102 & - & & & & And16 \\
\hline 16100.282 & 6.35 & -0.043 & & & & $\bullet$ & And16 \\
\hline 16102.408 & 5.87 & +0.546 & $\bullet$ & & $\bullet$ & & And16 \\
\hline 16125.899 & 6.35 & +0.860 & & - & & - & This work \\
\hline 16156.557 & 5.96 & -0.294 & $\bullet$ & & & $\bullet$ & And16 \\
\hline 16165.029 & 6.32 & +0.988 & - & $\bullet$ & & $\bullet$ & And16 \\
\hline 16171.930 & 6.38 & -0.445 & $\bullet$ & & $\bullet$ & $\bullet$ & And16 \\
\hline 16174.975 & 6.38 & +0.185 & - & - & - & $\bullet$ & And16 \\
\hline 16177.991 & 6.38 & -0.402 & & $\bullet$ & $\bullet$ & $\bullet$ & And16 \\
\hline 16179.583 & 6.32 & +0.261 & $\bullet$ & $\bullet$ & $\bullet$ & $\bullet$ & And16 \\
\hline 16180.900 & 6.28 & +0.295 & - & $\bullet$ & - & $\bullet$ & And16 \\
\hline 16182.170 & 6.32 & -0.708 & & & $\bullet$ & & This work \\
\hline 16185.799 & 6.39 & +0.264 & & $\bullet$ & & & This work \\
\hline 16195.060 & 6.39 & +0.467 & $\bullet$ & $\bullet$ & $\bullet$ & & And16 \\
\hline 16198.502 & 5.41 & -0.444 & - & - & - & $\bullet$ & And16 \\
\hline 16201.513 & 6.38 & -0.329 & - & & - & & This work \\
\hline 16207.744 & 6.32 & +0.585 & $\bullet$ & $\bullet$ & $\bullet$ & & And16 \\
\hline 16377.388 & 6.36 & -0.465 & - & & - & $\bullet$ & And16 \\
\hline 16384.141 & 6.36 & -0.736 & & & $\bullet$ & $\bullet$ & And16 \\
\hline 16394.389 & 5.96 & +0.358 & $\bullet$ & $\bullet$ & $\bullet$ & - & And16 \\
\hline 16396.306 & 6.28 & -0.530 & $\bullet$ & & & & This work \\
\hline 16404.601 & 6.36 & +0.581 & - & & $\bullet$ & & And16 \\
\hline 16407.786 & 6.29 & +0.007 & $\bullet$ & $\bullet$ & & & This work \\
\hline 16436.621 & 5.92 & +0.007 & $\bullet$ & & - & & And16 \\
\hline 16440.394 & 6.29 & -0.241 & - & & - & & And16 \\
\hline 16444.816 & 5.83 & +0.663 & $\bullet$ & - & - & & And16 \\
\hline 16466.921 & 6.39 & +0.003 & $\bullet$ & $\bullet$ & $\bullet$ & $\bullet$ & And16 \\
\hline 16471.753 & 6.37 & +0.030 & - & & & $\bullet$ & And16 \\
\hline 16474.077 & 6.02 & -0.959 & - & $\bullet$ & & • & And16 \\
\hline 16481.228 & 6.39 & -0.162 & $\bullet$ & & & & This work \\
\hline 16486.666 & 5.83 & +0.783 & - & & - & $\bullet$ & This work \\
\hline 16506.293 & 5.95 & -0.463 & & & - & & This work \\
\hline
\end{tabular}


Table A4 - continued

\begin{tabular}{|c|c|c|c|c|c|c|c|}
\hline \multirow{2}{*}{$\begin{array}{l}\lambda_{\text {air }} \\
[\AA]]\end{array}$} & \multirow{2}{*}{$\begin{array}{c}\chi_{\mathrm{I}} \\
{[\mathrm{eV}]}\end{array}$} & \multirow[t]{2}{*}{$\log g f$} & \multicolumn{4}{|c|}{ Line list ${ }^{a}$} & \multirow[t]{2}{*}{ Reference $^{b}$} \\
\hline & & & MRD & MPD & MRG & MPG & \\
\hline 16537.994 & 6.29 & -0.867 & - & & & & This work \\
\hline 16539.193 & 6.34 & -0.119 & - & & & & And16 \\
\hline 16544.667 & 6.34 & -0.029 & - & & - & - & And16 \\
\hline 16551.994 & 6.41 & +0.338 & $\bullet$ & & $\bullet$ & $\bullet$ & And16 \\
\hline 16557.148 & 6.41 & -1.083 & & & & $\bullet$ & This work \\
\hline 16559.677 & 6.40 & +0.210 & & & & - & And16 \\
\hline 16561.764 & 5.98 & +0.243 & & - & & & And16 \\
\hline 16586.051 & 5.62 & -0.753 & & & - & - & And16 \\
\hline 16612.761 & 6.40 & +0.286 & - & & $\bullet$ & & And16 \\
\hline 16629.836 & 6.57 & -0.435 & $\bullet$ & & & & This work \\
\hline 16645.874 & 5.96 & -0.032 & - & - & - & - & And16 \\
\hline 16807.435 & 5.83 & -1.301 & & & - & & And16 \\
\hline 16833.052 & 5.96 & -0.889 & - & & - & $\bullet$ & And16 \\
\hline 16843.228 & 5.87 & -1.321 & - & & & & And16 \\
\hline 16865.513 & 6.41 & -0.749 & & & & - & And16 \\
\hline 16869.950 & 6.41 & -0.415 & & & - & & This work \\
\hline 16874.116 & 6.35 & -0.159 & - & & & & And16 \\
\hline 16892.384 & 6.31 & -0.799 & $\bullet$ & & - & - & This work \\
\hline 16969.910 & 5.95 & -0.069 & & & $\bullet$ & & This work \\
\hline 17005.450 & 6.07 & +0.005 & & $\bullet$ & & - & This work \\
\hline 17008.971 & 6.62 & -0.301 & - & & - & & This work \\
\hline 17011.095 & 5.95 & +0.102 & $\bullet$ & & & - & This work \\
\hline 17037.787 & 6.39 & -0.852 & & & & - & And16 \\
\hline
\end{tabular}

${ }^{a}$ Line lists. MRD: metal-rich dwarfs; MPD: metal-poor dwarfs; MRG: metal-rich giants; MPG: metal-poor giants.

${ }^{b}$ References. Sou08: Sousa et al. (2008); And16: Andreasen et al. (2016).

Table A5. Merged Fe II line lists.

\begin{tabular}{|c|c|c|c|c|c|c|c|}
\hline \multirow{2}{*}{$\begin{array}{l}\lambda_{\text {air }} \\
[\AA]]\end{array}$} & \multirow{2}{*}{$\begin{array}{c}\chi_{\mathrm{II}} \\
{[\mathrm{eV}]}\end{array}$} & \multirow[t]{2}{*}{$\log g f$} & \multicolumn{4}{|c|}{ Line list ${ }^{a}$} & \multirow[t]{2}{*}{ Reference $^{b}$} \\
\hline & & & MRD & MPD & MRG & MPG & \\
\hline 5325.552 & 3.22 & -3.160 & & $\bullet$ & & $\bullet$ & Sou08 \\
\hline 5414.070 & 3.22 & -3.580 & & & $\bullet$ & & Sou08 \\
\hline 5425.248 & 3.20 & -3.220 & - & • & $\bullet$ & • & Sou08 \\
\hline 5534.838 & 3.24 & -2.730 & & $\bullet$ & & & Sou08 \\
\hline 5991.371 & 3.15 & -3.540 & - & & & & Sou08 \\
\hline 6084.102 & 3.20 & -3.780 & $\bullet$ & & $\bullet$ & & Sou08 \\
\hline 6149.246 & 3.89 & -2.720 & $\bullet$ & & $\bullet$ & • & Sou08 \\
\hline 6238.386 & 3.89 & -2.754 & & - & - & & Sou08 \\
\hline 6247.557 & 3.89 & -2.310 & & - & & & Sou08 \\
\hline 6369.459 & 2.89 & -4.160 & $\bullet$ & & & $\bullet$ & Sou08 \\
\hline 6416.919 & 3.89 & -2.650 & $\bullet$ & & & & Sou08 \\
\hline 6432.676 & 2.89 & -3.520 & $\bullet$ & $\bullet$ & $\bullet$ & & Sou08 \\
\hline 6456.380 & 3.90 & -2.100 & & - & - & $\bullet$ & Sou08 \\
\hline 6516.077 & 2.89 & -3.320 & $\bullet$ & $\bullet$ & & & Sou08 \\
\hline 7222.391 & 3.89 & -3.360 & $\bullet$ & $\bullet$ & $\bullet$ & & This work \\
\hline 7224.478 & 3.89 & -3.240 & - & $\bullet$ & - & & This work \\
\hline 7449.329 & 3.89 & -3.090 & - & & - & & This work \\
\hline 7479.693 & 3.89 & -3.680 & - & & $\bullet$ & & This work \\
\hline 7515.830 & 3.90 & -3.460 & - & & - & & This work \\
\hline 7533.368 & 3.90 & -3.600 & $\bullet$ & & - & & This work \\
\hline 7711.720 & 3.90 & -2.500 & - & - & - & - & This work \\
\hline 9997.598 & 5.48 & -1.867 & $\bullet$ & & - & & This work \\
\hline 10501.503 & 5.55 & -2.086 & - & & & & And16 \\
\hline
\end{tabular}

${ }^{a}$ Line lists. MRD: metal-rich dwarfs; MPD: metal-poor dwarfs; MRG: metal-rich giants; MPG: metal-poor giants.

${ }^{b}$ References. Sou08: Sousa et al. (2008); And16: Andreasen et al. (2016). 

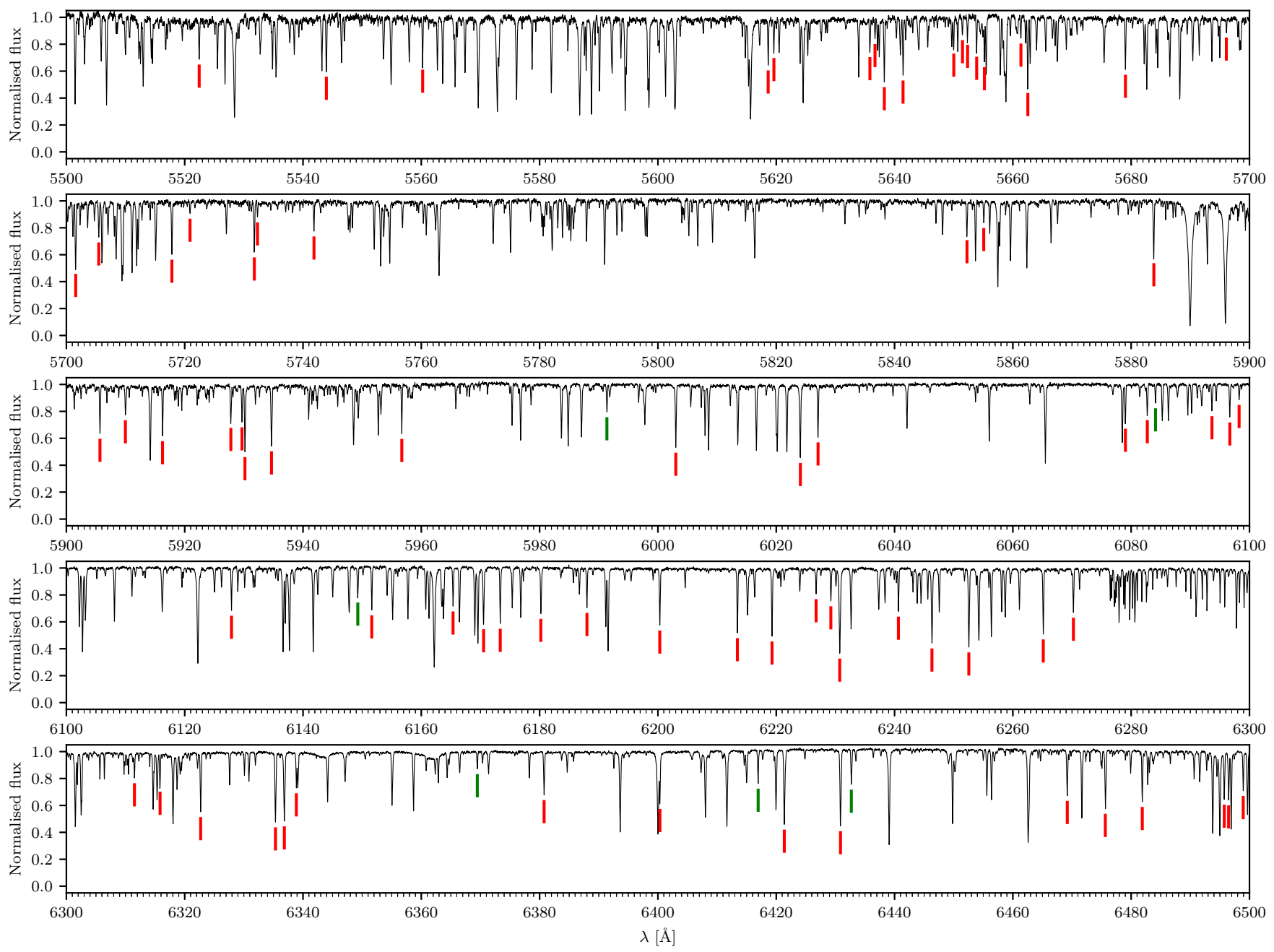

Figure A1. CARMENES spectrum of $18 \mathrm{Sco}$. Fe I and Fe II lines are shown in red and green, respectively. 

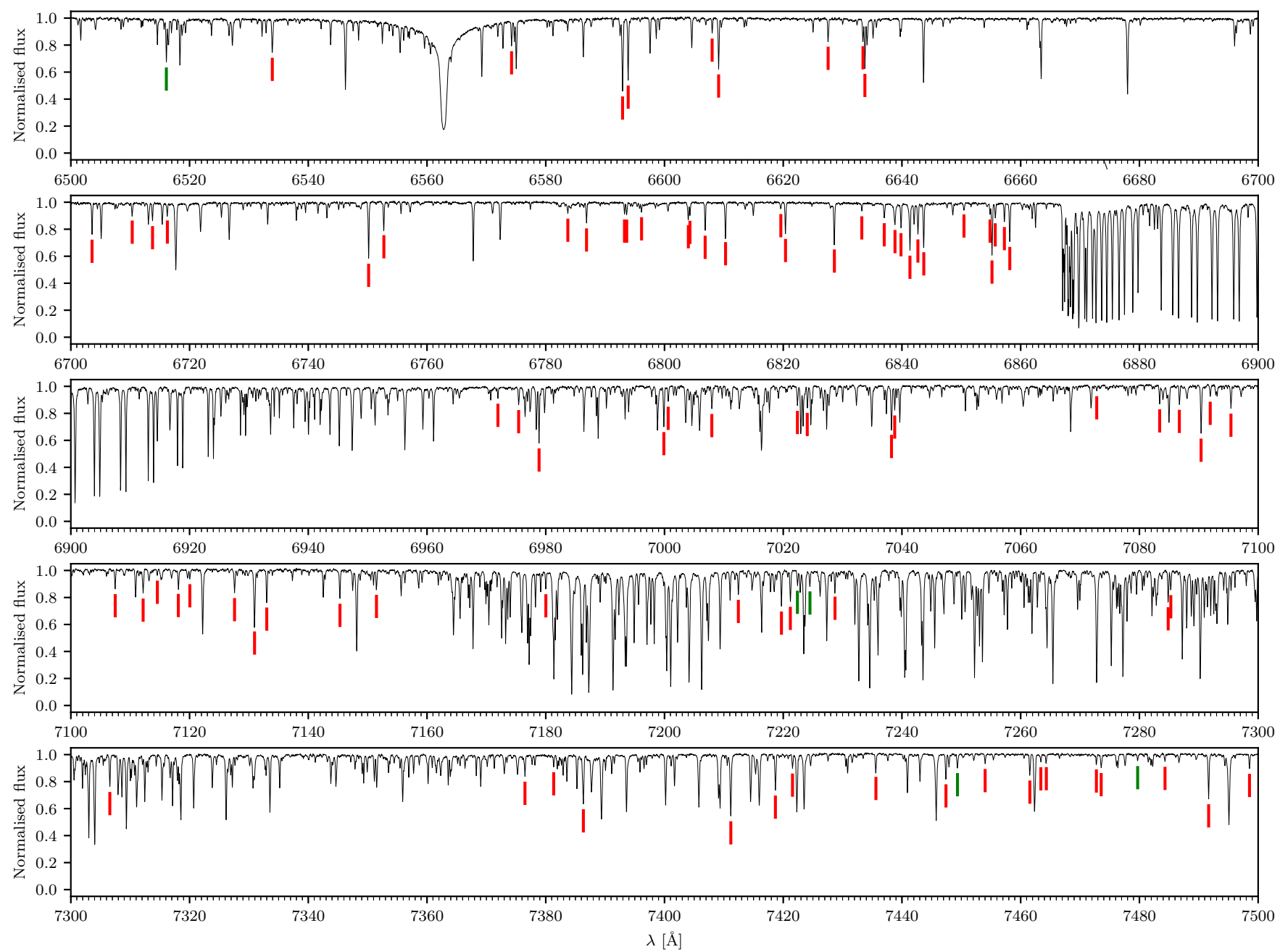

Figure A1. continued 

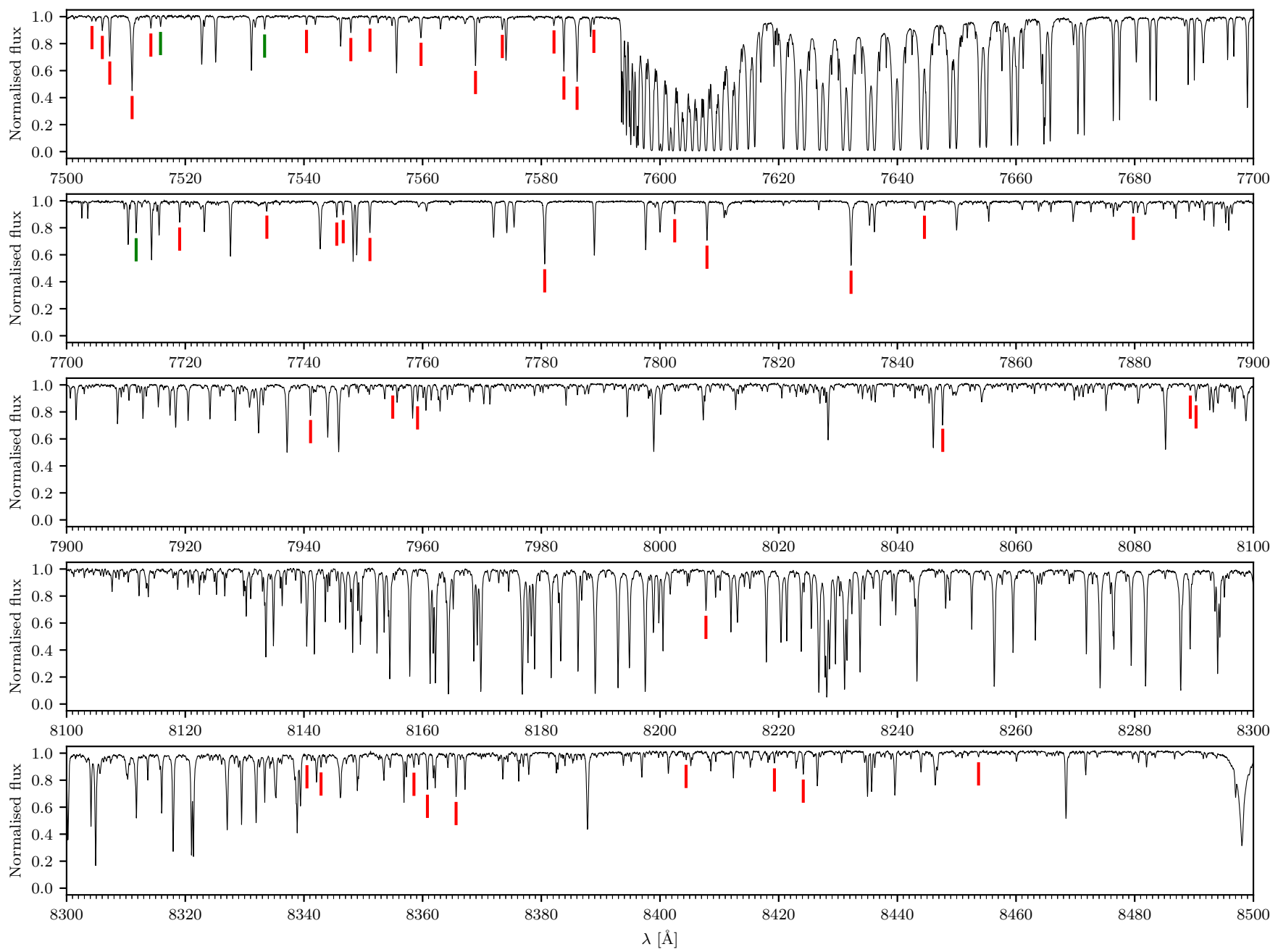

Figure A1. continued 

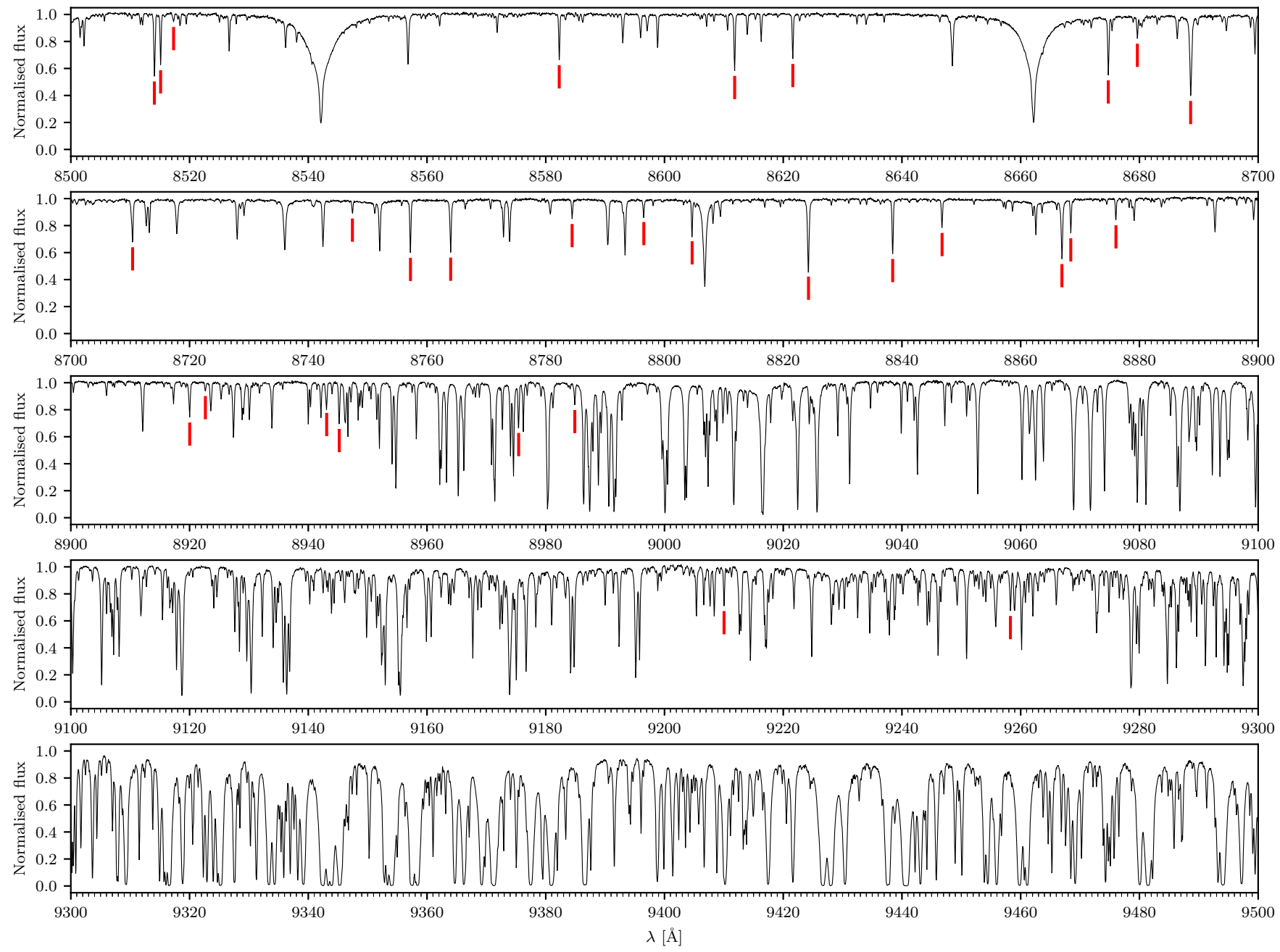

Figure A1. continued 

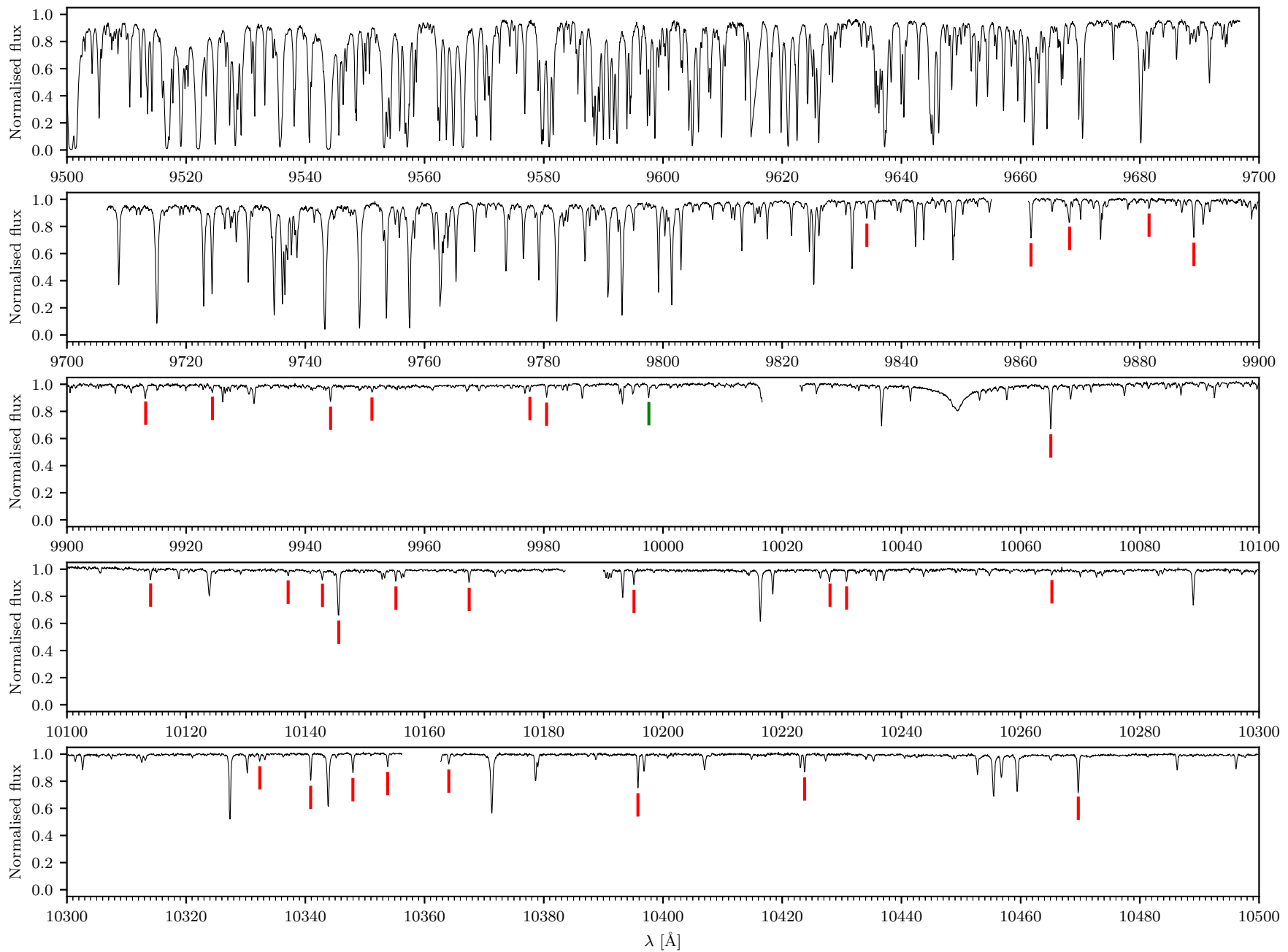

Figure A1. continued 

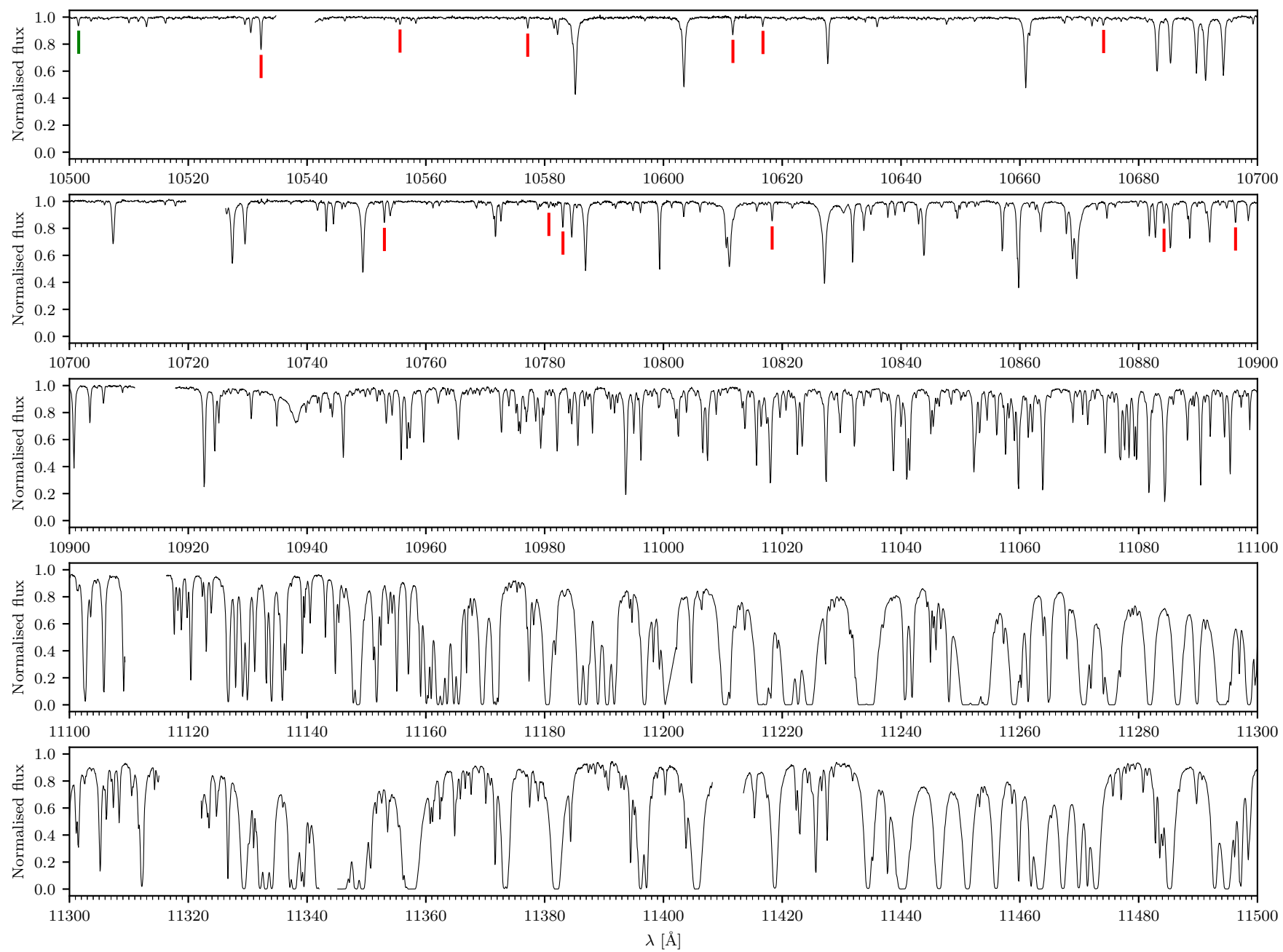

Figure A1. continued 

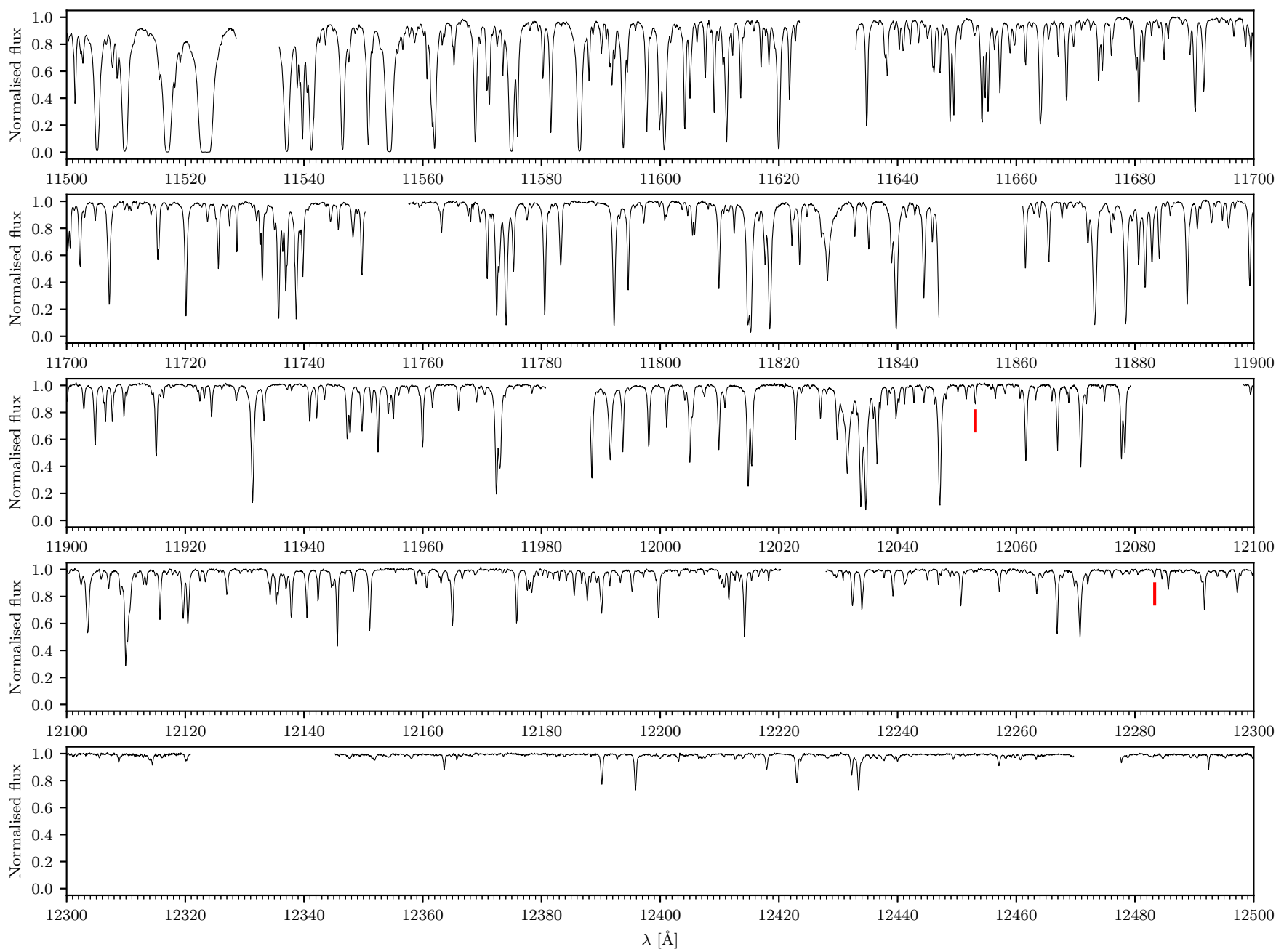

Figure A1. continued 

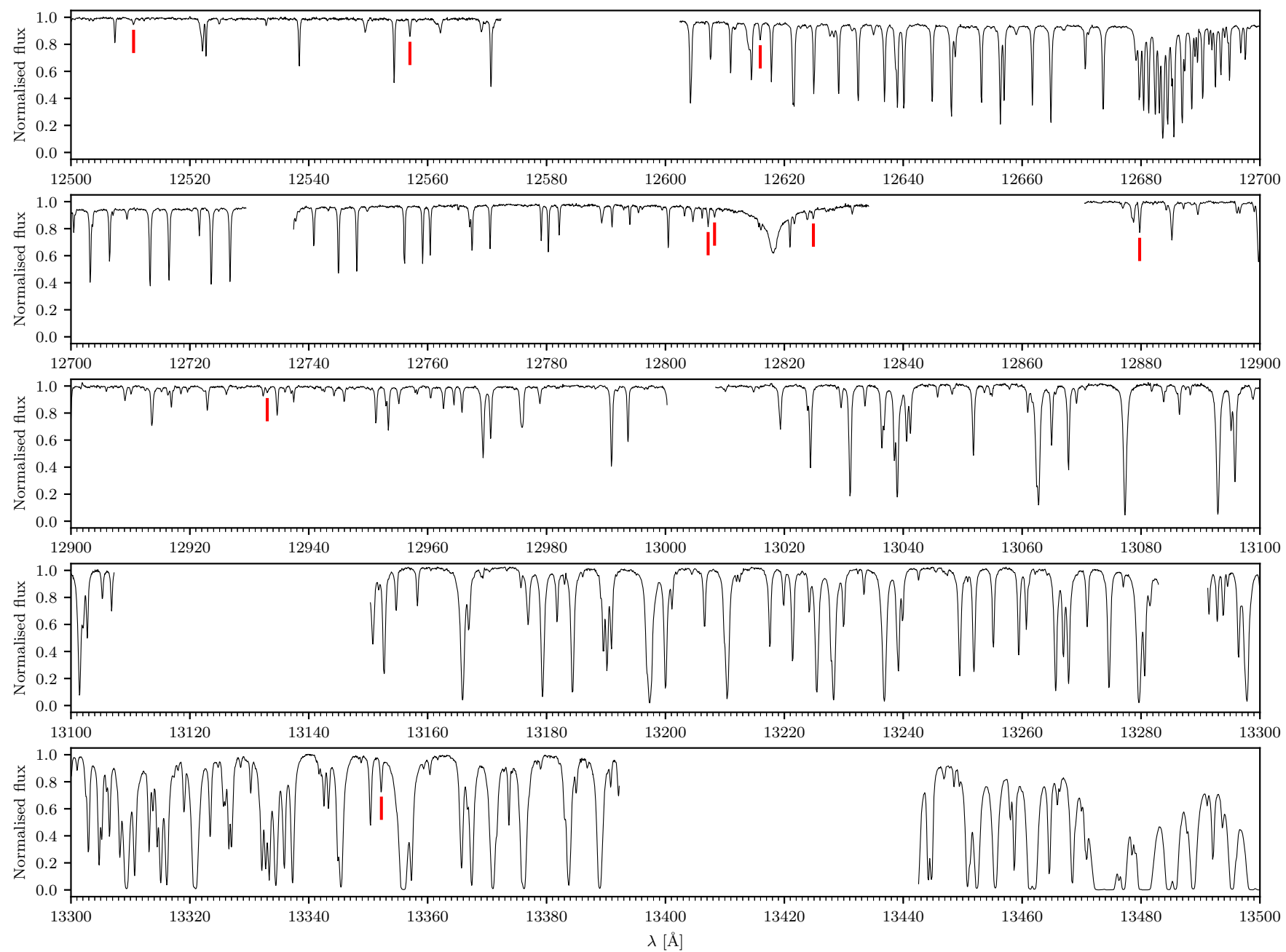

Figure A1. continued 

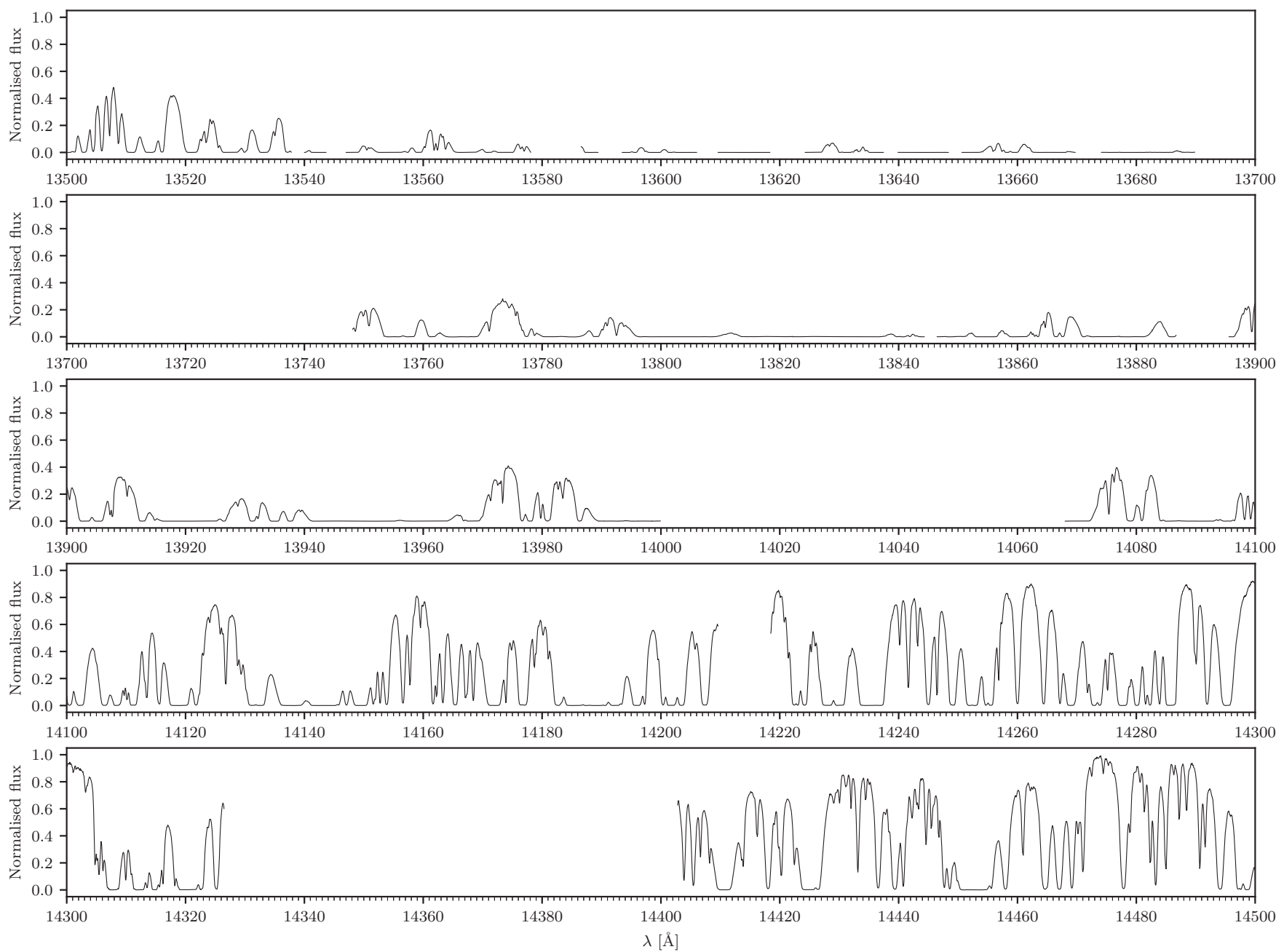

Figure A1. continued 

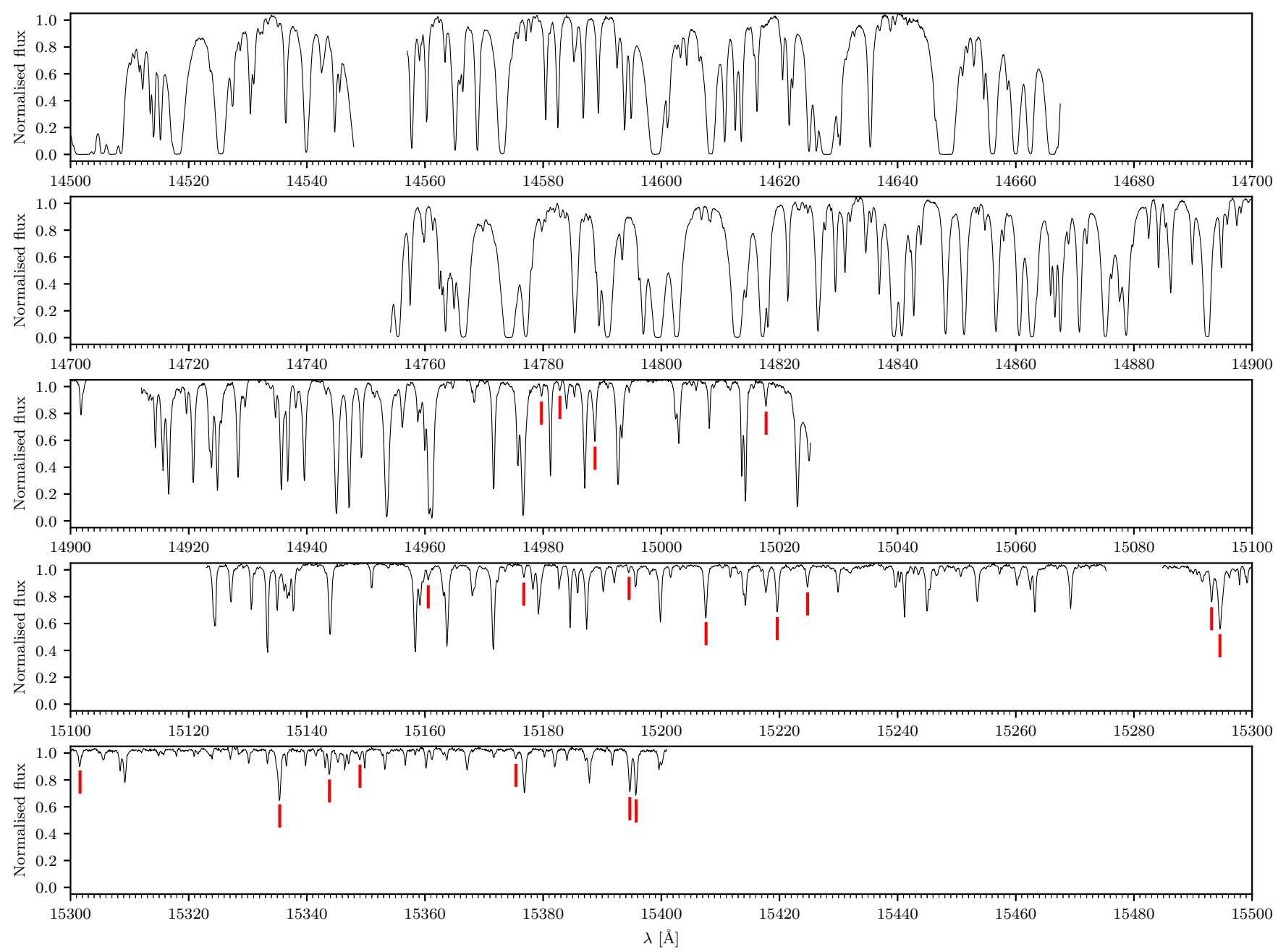

Figure A1. continued 

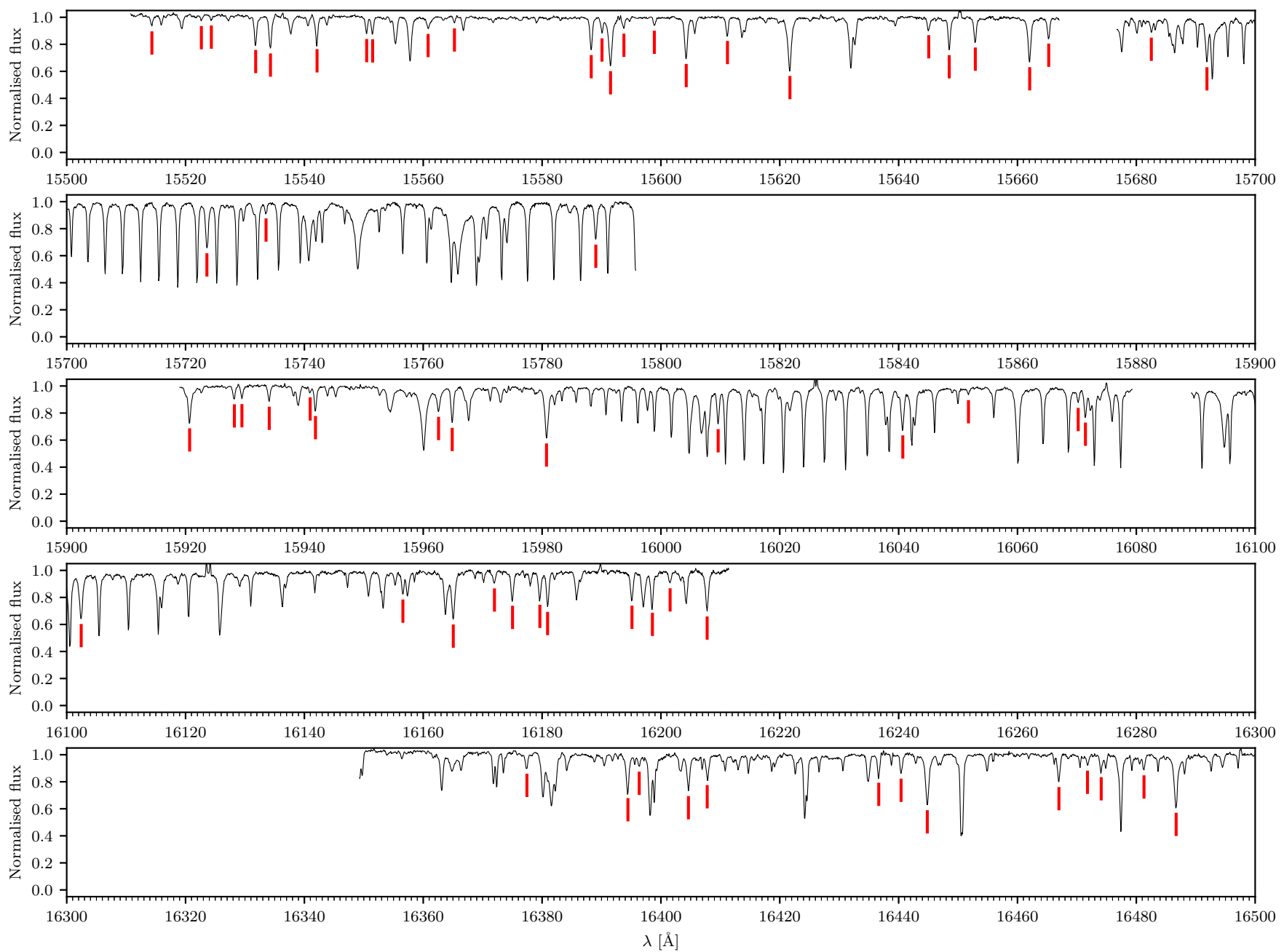

Figure A1. continued 

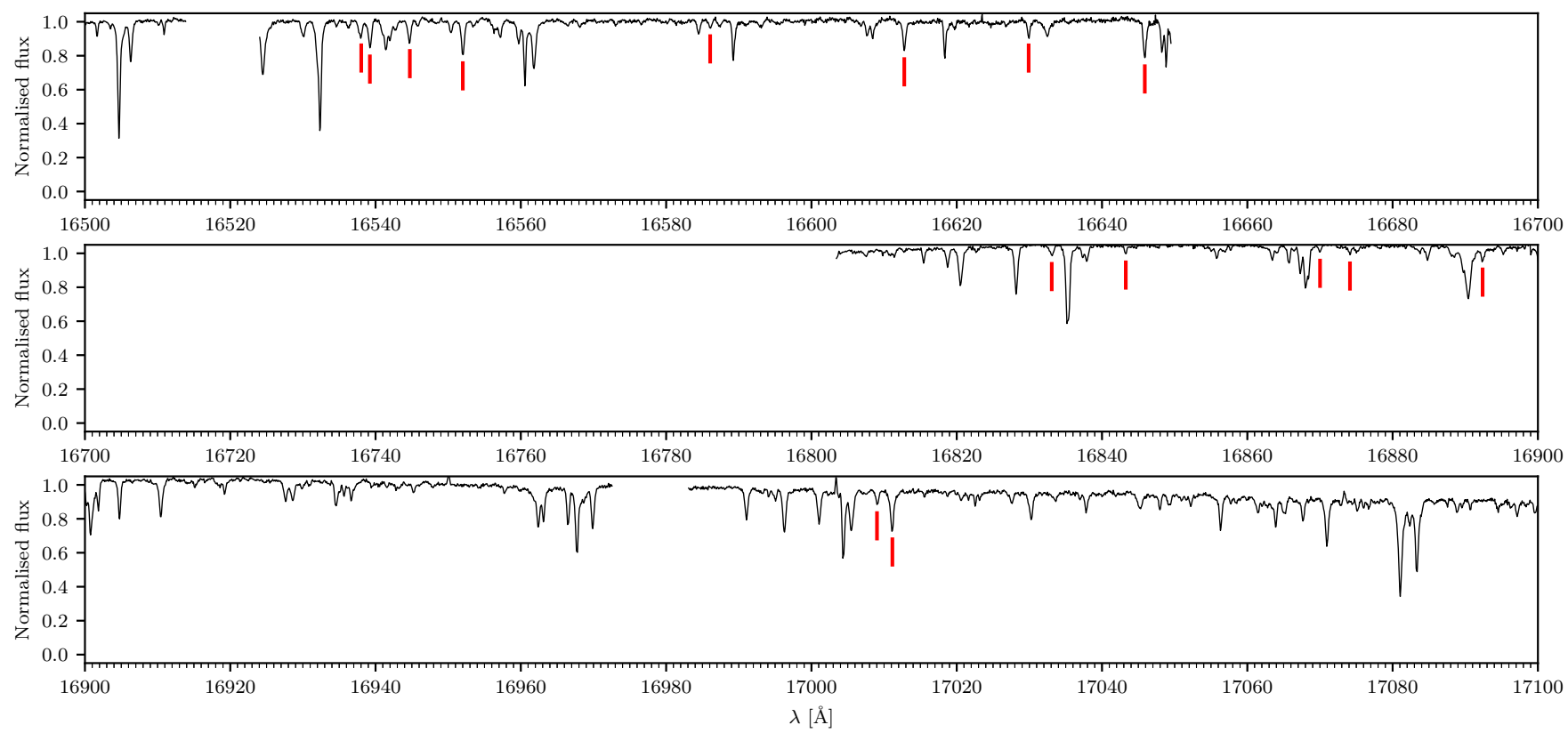

Figure A1. continued

This paper has been typeset from a $\mathrm{T}_{\mathrm{E}} \mathrm{X} / \mathrm{LT} \mathrm{E} \mathrm{X}$ file prepared by the author. 